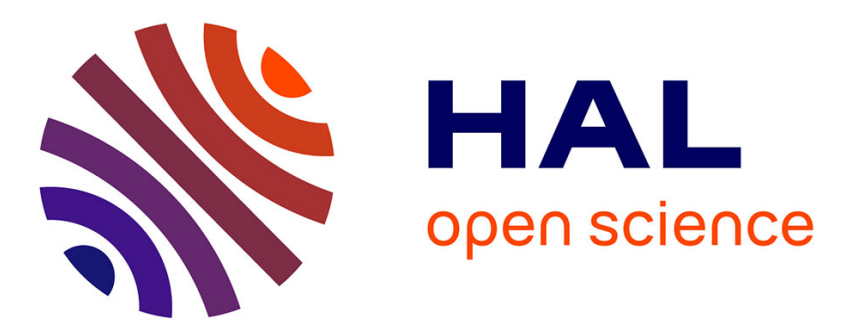

\title{
Synthesis of l-arabinopyranose containing hederagenin saponins
}

Karen Plé, Martin Chwalek, Laurence Voutquenne-Nazabadioko

\section{To cite this version:}

Karen Plé, Martin Chwalek, Laurence Voutquenne-Nazabadioko. Synthesis of l-arabinopyranose containing hederagenin saponins. Tetrahedron, 2005, 61 (18), pp.4347-4362. hal-01996917

\section{HAL Id: hal-01996917 \\ https://hal.univ-reims.fr/hal-01996917}

Submitted on 22 Sep 2021

HAL is a multi-disciplinary open access archive for the deposit and dissemination of scientific research documents, whether they are published or not. The documents may come from teaching and research institutions in France or abroad, or from public or private research centers.
L'archive ouverte pluridisciplinaire HAL, est destinée au dépôt et à la diffusion de documents scientifiques de niveau recherche, publiés ou non, émanant des établissements d'enseignement et de recherche français ou étrangers, des laboratoires publics ou privés. 
Synthesis of L-arabinopyranose containing hederagenin saponins

Karen Plé*, Martin Chwalek and Laurence Voutquenne-Nazabadioko

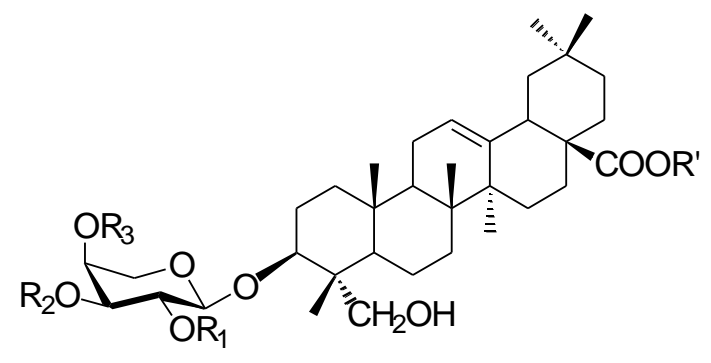

$\mathrm{R}_{1}, \mathrm{R}_{2}, \mathrm{R}_{3}=\mathrm{H}, \beta$-D-Xyl, $\beta$-D-Glc

$\mathrm{R}^{\prime}=\mathrm{H}, \mathrm{CH}_{3}$

The synthesis of eight L-arabinopyranose containing hederagenin saponins, five of which are natural products, and their methyl esters is described. 


\title{
Synthesis of L-arabinopyranose containing hederagenin saponins
}

Karen Plé*, Martin Chwalek and Laurence Voutquenne-Nazabadioko

CNRS FRE 2715 Isolement, Structure, Transformations et Synthèse de Substances Naturelles. Université de Reims Champagne-Ardenne, IFR 53 Biomolécules, CPCBAI, Bât. 18, Moulin de la Housse, BP 1039, 51687 Reims, France

\begin{abstract}
The synthesis of eight hederagenin saponins, five of which are natural products, and their methyl esters is described as part of an ongoing study of the biological activity of triterpenoid saponins. Six disaccharides consisting of an L-arabinopyranose glycosylated in positions 2, 3, or 4 with a $\beta$-D-xylopyranose or a $\beta$-D-glucopyranose residue respectively, were synthesized in good to excellent yields. The saponins were then prepared in good yields through glycosylation with a suitably protected hederagenin derivative followed by total deprotection and treatment with diazomethane.
\end{abstract}

\section{Introduction}

Saponins are triterpene or steroid glycosides which are found in a wide variety of plants and certain marine organisms. ${ }^{1}$ Interest in saponins is rapidly increasing due to their numerous biological properties, ${ }^{2,3}$ but a limiting factor in their evaluation is often the small amounts obtained from natural product extraction. Chemical synthesis offers an alternative to saponin extraction in plants, and opens the door to the preparation of tailor-made molecules. In the study of saponin structureactivity relationships, both the aglycone and the sugar moiety play an important role in the evaluation of biological activity and must be considered individually. It is thus essential to have access to all the positional isomers of a given sugar moiety while keeping the aglycone constant. We have implemented this strategy as part of our ongoing study of the hemolytic activity of hemisynthetic hederagenin saponins in an attempt to better understand the role of the sugar moiety on hemolysis. ${ }^{4}$ Having previously synthesized $\alpha$-hederin and its positional isomers with respect to the L-rhamnopyranosyl-L-arabinopyranose disaccharide moiety ${ }^{5}$ the synthesis of two additional families of hederagenin saponins was undertaken (Figure 1). While keeping an L-arabinopyranose as the first sugar, a D-xylopyranose and a D-glucopyranose were chosen as the second one in the disaccharide moiety. In total, we wished to synthesize eight hederagenin saponins including 2 and $\mathbf{6}$ having a $\beta$ configuration between the aglycone and the sugar chain, normally not found in natural sources. Five

\footnotetext{
* Corresponding author. Tel.: +33-3-26-91-84-19; fax: +33-3-26-91-35-96; e-mail: karen.ple@univ-reims.fr
} 
of the eight saponins are naturally occurring in plants $(\mathbf{1}, \mathbf{3}, \mathbf{5}, \mathbf{7}, \mathbf{8}),{ }^{1,6}$ and several of the saponins have shown molluscicidal ${ }^{6 \mathrm{~b}, 7}(\mathbf{3}, \mathbf{5})$, hemolytic $^{8}(\mathbf{5})$, and cytotoxic ${ }^{9}(\mathbf{5}, 7)$ activities.

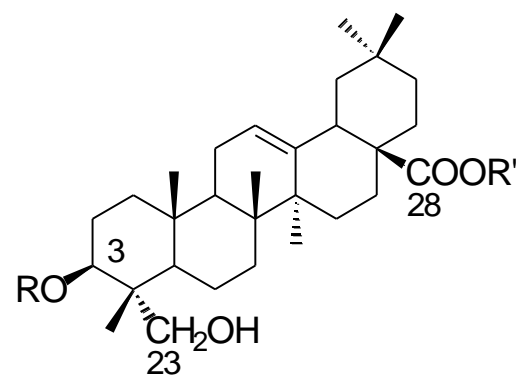

\begin{tabular}{|c|c|c|c|}
\hline $\mathrm{R}=\beta-\mathrm{D}-\mathrm{Xyl}-(1 \rightarrow 2)-\alpha-\mathrm{L}-\mathrm{Ara}$ & $\begin{array}{l}\mathrm{R}^{\prime}=\mathrm{H}(\mathbf{1}) \\
\mathrm{R}^{\prime}=\mathrm{CH}_{3}(\mathbf{1 a})\end{array}$ & $\mathrm{R}=\beta-\mathrm{D}-\mathrm{Glc}-(1 \rightarrow 2)-\alpha-\mathrm{L}-\mathrm{Ara}$ & $\begin{array}{l}\mathrm{R}^{\prime}=\mathrm{H}(\mathbf{5}) \\
\mathrm{R}^{\prime}=\mathrm{CH}_{3}(\mathbf{5 a})\end{array}$ \\
\hline $\mathrm{R}=\beta-\mathrm{D}-\mathrm{Xyl}-(1 \rightarrow 2)-\beta-\mathrm{L}-\mathrm{Ara}$ & $\begin{array}{l}\mathrm{R}^{\prime}=\mathrm{H}(\mathbf{2}) \\
\mathrm{R}^{\prime}=\mathrm{CH}_{3}(\mathbf{2 a})\end{array}$ & $\mathrm{R}=\beta-\mathrm{D}-\mathrm{Glc}-(1 \rightarrow 2)-\beta-\mathrm{L}-\mathrm{Ara}$ & $\begin{array}{l}\mathrm{R}^{\prime}=\mathrm{H}(\mathbf{6}) \\
\mathrm{R}^{\prime}=\mathrm{CH}_{3}(\mathbf{6 a})\end{array}$ \\
\hline $\mathrm{R}=\beta-\mathrm{D}-\mathrm{Xyl}-(1 \rightarrow 3)-\alpha-\mathrm{L}-\mathrm{Ara}$ & $\begin{array}{l}\mathrm{R}^{\prime}=\mathrm{H}(\mathbf{3}) \\
\mathrm{R}^{\prime}=\mathrm{CH}_{3}(\mathbf{3 a})\end{array}$ & $\mathrm{R}=\beta-\mathrm{D}-\mathrm{Glc}-(1 \rightarrow 3)-\alpha-\mathrm{L}-\mathrm{Ara}$ & $\begin{array}{l}\mathrm{R}^{\prime}=\mathrm{H}(\mathbf{7}) \\
\mathrm{R}^{\prime}=\mathrm{CH}_{3}(\mathbf{7 a})\end{array}$ \\
\hline $\mathrm{R}=\beta-\mathrm{D}-\mathrm{Xyl}-(1 \rightarrow 4)-\alpha-\mathrm{L}-\mathrm{Ara}$ & $\begin{array}{l}\mathrm{R}^{\prime}=\mathrm{H}(\mathbf{4}) \\
\mathrm{R}^{\prime}=\mathrm{CH}_{3}(\mathbf{4 a})\end{array}$ & $\mathrm{R}=\beta-\mathrm{D}-\mathrm{Glc}-(1 \rightarrow 4)-\alpha-\mathrm{L}-\mathrm{Ara}$ & $\begin{array}{l}\mathrm{R}^{\prime}=\mathrm{H}(\mathbf{8}) \\
\mathrm{R}^{\prime}=\mathrm{CH}_{3}(\mathbf{8 a})\end{array}$ \\
\hline
\end{tabular}

Figure 1. D-Xyl-L-Ara and D-Glc-L-Ara hederagenin saponins

While the synthesis of steroidal saponins has been widely reported in the literature, ${ }^{10}$ that of triterpenoid saponins has attracted less attention. A large majority of triterpenoid saponin syntheses involve oleanolic acid as the aglycone, ${ }^{11}$ and the use of others remains rare (e.g. hederagenin, ${ }^{5}$ glycyrrhetic acid, ${ }^{12}$ ursolic acid, ${ }^{13}$ or medicagenic acid $\left.{ }^{14}\right)$.

Few examples exist in the literature concerning the synthesis of disaccharides with an Larabinopyranose at the reducing end. In the $\beta$-D-xylopyranosyl- $\alpha$-L-arabinopyranose series, several syntheses have been reported for the disaccharide portion of the saponin OSW-1 ( $\beta$-D-Xyl- $(1 \rightarrow 3)-$ $\alpha$-L-Ara). ${ }^{15}$ Deng et al. originally reported the use of a benzyl L-arabinopyranose precursor with free hydroxyl groups in positions 3 and 4 and a trichloroacetimidate derivative of $\beta$-D-xylopyranose giving a mixture of disaccharides with glycosylation in position 3 being the predominant reaction. ${ }^{15 \mathrm{e}}$ The same authors also reported that glycosylation of a phenyl 1-thio-L-arabinopyranose precursor with free hydroxyl groups in positions 3 and 4 resulted in the $\beta$-D-Xyl-(1 $\rightarrow 4)$ - $\alpha$-L-Ara disaccharide as the major reaction product. ${ }^{16}$ In the synthesis of $\mathrm{Yu}$ et al. ${ }^{15 \mathrm{c}}$ the target disaccharide $\beta$-D-Xyl-(1 $\rightarrow 3)$ - $\alpha$-L-Ara was prepared in $93 \%$ yield from a protected L-arabinopyranose derivative and a D-xylopyranosyl trichloroacetimidate.

In the $\beta$-D-glucopyranose- $\alpha$-L-arabinopyranose series, Liptak et al. described the first synthesis of a $\beta$-D-glucopyranosyl-( $(1 \rightarrow 2)$ - $\alpha$-L-arabinopyranose disaccharide derivative in 1982 as part of a ${ }^{13} \mathrm{C}$ NMR spectroscopy study of methyl and benzyl $\beta$-L-arabinopyranose oligosaccharides. ${ }^{17}$ The 
disaccharide was synthesized in low yield using a suitably protected benzyl $\beta$-L-arabinopyranose derivative and an acetobromoglucose in the presence of an excess of mercury cyanide. More recently, Field et al. reported the synthesis of two D-glucopyranose-L-arabinopyranose disaccharides which are fragments of the oat root saponin Avenacin A-1. ${ }^{18}$ The desired disaccharides were synthesized in good yields using a thioglycoside donor.

We wish to describe here the efficient synthesis of $\beta$-D-xylopyranosyl- $\alpha$-L-arabinopyranose and $\beta$ D-glucopyranosyl- $\alpha$-L-arabinopyranose disaccharides and their use in the synthesis of eight hederagenin saponins and their methyl esters as part of a directed study of the hemolytic activity of

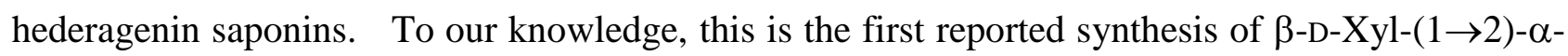
L-Ara and $\beta$-D-Glc- $(1 \rightarrow 3)-\alpha$-L-Ara disaccharides as well as that of eight hederagenin saponins, five of which are natural products.

\section{Results and Discussion}

The previously described L-arabinopyranose derivatives $\mathbf{9 , 1 0}$ and $\mathbf{1 1}$ were the starting point for the disaccharide syntheses. $^{5}$ In the D-xylopyranose series, glycosylation with 2,3,4-tri- $O$-benzoyl- $\alpha$-Dxylopyranosyl trichloroacetimidate ${ }^{19}(\mathbf{1 2})$ gave the desired disaccharides in good to excellent yields (Scheme 1).
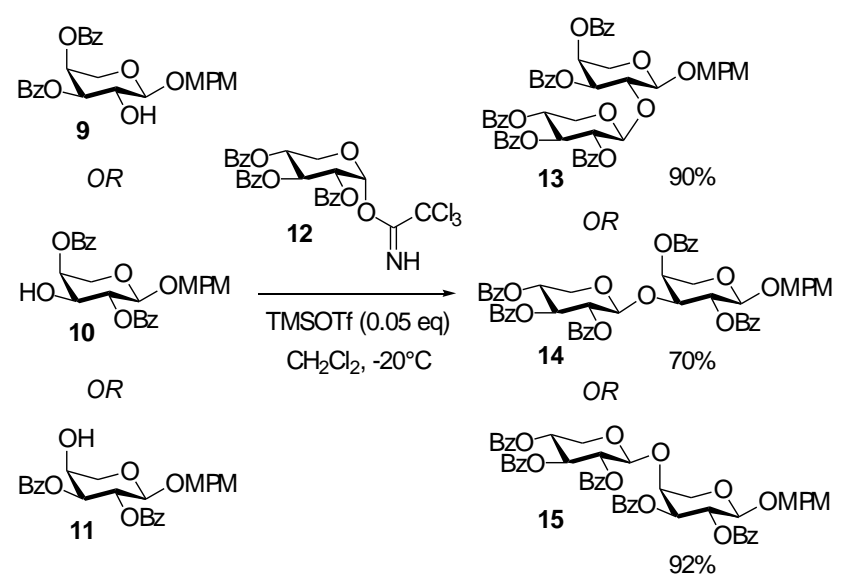

\section{Scheme 1}

When the same glycosylation strategy was tried in the D-glucopyranose series using 2,3,4,6-tetra- $O$ benzoyl- $\alpha$-D-glucopyranosyl trichloroacetimidate $(\mathbf{1 6}),{ }^{20}$ the reaction resulted in the isolation of the corresponding orthoesters in good yields (75\%-92\%) with no trace of the desired disaccharides (Scheme 2). Modifying the reaction conditions or attempting orthoester opening with excess TMSOTf or $\mathrm{HgBr}_{2}$ resulted in total degradation of the starting material. The use of the corresponding per- $O$-benzoylated thioglycoside donor $17^{21}$ was also tried with the arabinopyranose acceptor 10. Once again, the orthoester was isolated in good yield. 


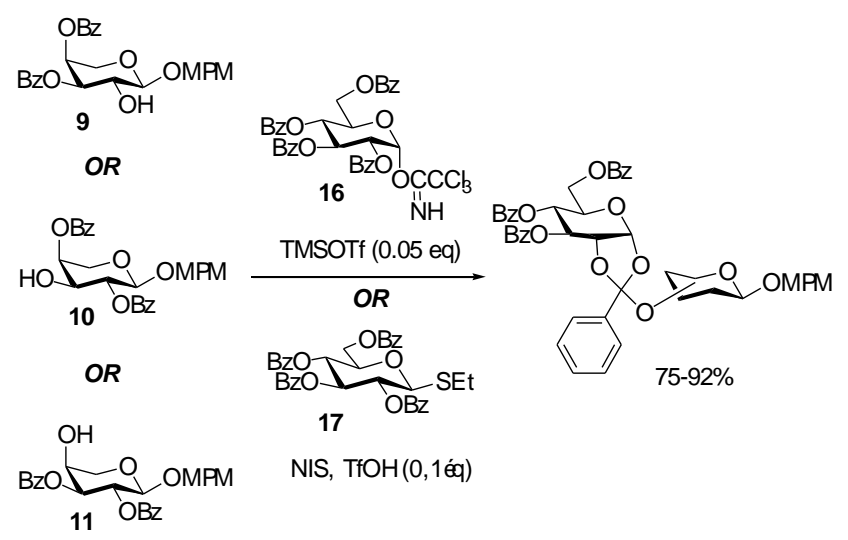

\section{Scheme 2}

Ethyl 2- $O$-benzoyl-3,4,6-tetra- $O$-benzyl-1-thio- $\beta$-D-glucopyranoside (22) was then synthesized using an analogous procedure described for D-galactose (Scheme 3). ${ }^{22}$ By replacing the benzoate protecting groups in positions 3, 4, and 6 with benzyl groups we hoped to enhance the reactivity of the thioglycoside donor, and avoid, if possible, further orthoester formation.
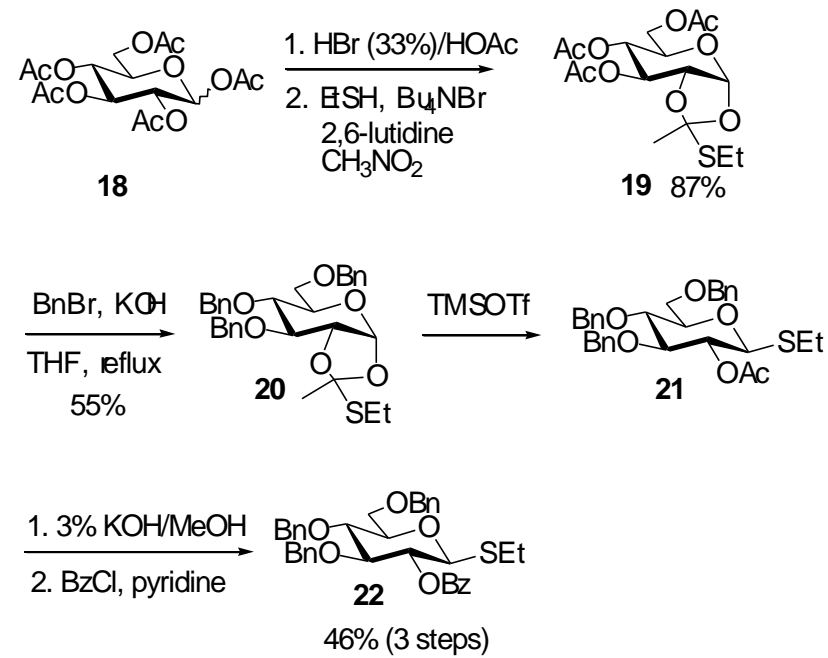

Scheme 3

Compound 22 was then successfully used as a donor in the glycosylation reactions with the arabinopyranose acceptors $\mathbf{9}, \mathbf{1 0}$, and 11. The disaccharides were obtained in good to moderate yields with no detectable orthoesters in the reaction mixtures (Scheme 4). 


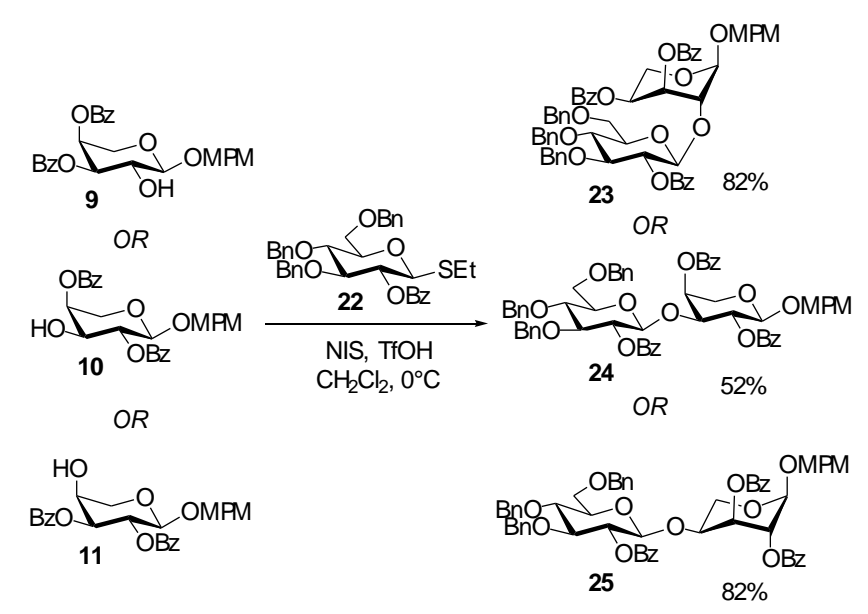

Scheme 4

One possible explanation for the moderate yield of disaccharide $\mathbf{2 4}$ could be the steric hindrance created by the addition of a D-glucopyranose in position 3 of the L-arabinopyranose derivative $\mathbf{1 0}$. Optimization of the glycosylation reaction at different temperatures or by the addition of a larger quantity of the thioglycoside donor (up to 3 equivalents) did not increase the yield.

The anomeric MPM protecting groups of the disaccharides (13-15, 23-25) were then removed in the presence of aqueous trifluoroacetic acid at room temperature. The resulting hemiacetal was reacted with trichloroacetonitrile in the presence of DBU, giving good to excellent yields of the corresponding trichloroacetimidates (Table 1).

Table 1. Trichloroacetimidate formation

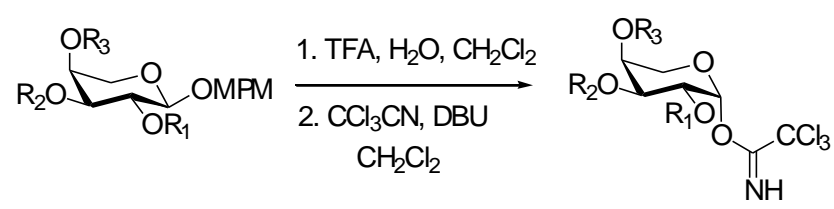

\begin{tabular}{cccccc} 
Disaccharide & $\mathrm{R}_{1}$ & $\mathrm{R}_{2}$ & $\mathrm{R}_{3}$ & Trichloroacetimidate & Yield \\
\hline $\mathbf{1 3}$ & $\mathbf{X y l}^{\mathrm{a}}$ & $\mathrm{OBz}$ & $\mathrm{Bz}$ & $\mathbf{2 7}$ & $89 \%$ \\
$\mathbf{1 4}$ & $\mathrm{Bz}$ & $\mathbf{X y l}$ & $\mathrm{Bz}$ & $\mathbf{2 8}$ & $80 \%$ \\
$\mathbf{1 5}$ & $\mathrm{Bz}$ & $\mathrm{Bz}$ & $\mathbf{X y l}$ & $\mathbf{2 9}$ & $83 \%$ \\
$\mathbf{2 3}$ & $\mathbf{G l c}^{\mathrm{b}}$ & $\mathrm{Bz}$ & $\mathrm{Bz}$ & $\mathbf{3 0}$ & $78 \%$ \\
$\mathbf{2 4}$ & $\mathrm{Bz}$ & $\mathbf{G l c}$ & $\mathrm{Bz}$ & $\mathbf{3 1}$ & $74 \%$ \\
$\mathbf{2 5}$ & $\mathrm{Bz}$ & $\mathrm{Bz}$ & $\mathbf{G l c}$ & $\mathbf{3 2}$ & $77 \%$ \\
\hline${ }^{\mathrm{a}} \mathbf{X y l}=(2,3,4-$-tri- $O$-benzoyl- $\beta$-D-xylopyranosyl) \\
${ }^{\mathrm{b}} \mathbf{G l c}=(2-O$-benzoyl-3,4,6-tri- $O$-benzyl- $\beta$-D-glucopyranosyl $)$
\end{tabular}

Saponin synthesis was then performed with the activated disaccharides $\mathbf{2 7 - 3 2}$ and the previously described allyl hederagenate derivative 33. ${ }^{5}$ Coupling at low temperature in the presence of a catalytic amount of TMSOTf gave the protected saponins in excellent yields (Table 2). 
Table 2. Hederagenin glycosylation

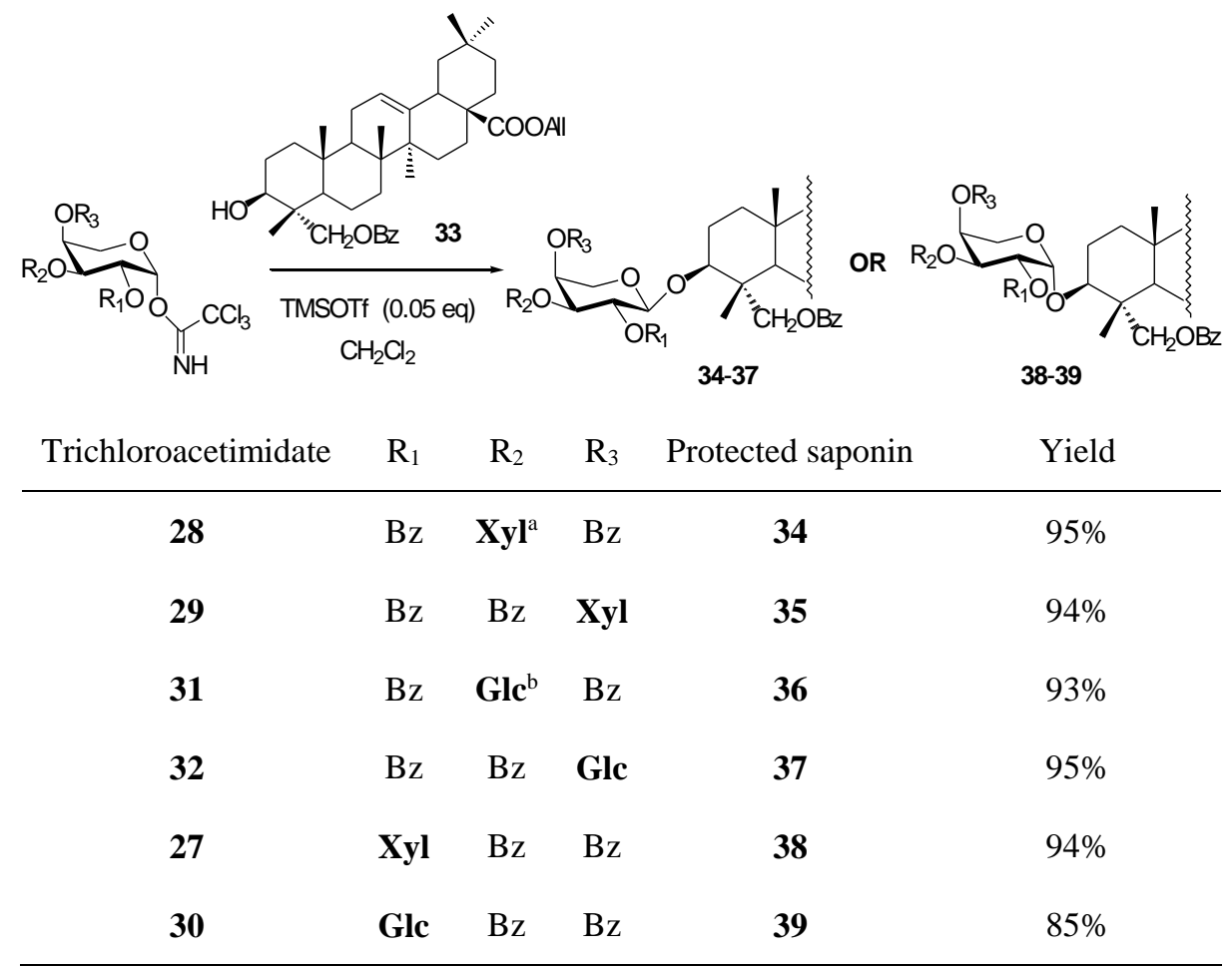

${ }^{\mathrm{a}} \mathbf{X y l}=(2,3,4-$ tri- $O$-benzoyl- $\beta$-D-xylopyranosyl $)$

${ }^{\mathrm{b}} \mathbf{G l c}=(2-O$-benzoyl-3,4,6-tri- $O$-benzyl- $\beta$-D-glucopyranosyl $)$

As expected, the presence of a benzoate in position 2 of the L-arabinopyranose moiety directed the glycosylation reaction and gave exclusive formation of the $\alpha$ anomers as a result of neighboring group participation. For the trichloroacetimidates $\mathbf{2 7}$ and $\mathbf{3 0}$ possessing a sugar residue in position 2 , the $\beta$ anomers were isolated as the major reaction products.

To prepare the corresponding $\alpha$ anomers of these two saponins, the coupling reaction was carried out in acetonitrile. Use of this solvent is known to promote equatorial bond formation in glycoside synthesis when neighboring group participation is absent. ${ }^{23}$ Glycosylation with 2 equivalents of the donor in acetonitrile at $-35^{\circ} \mathrm{C}$ gave a mixture of anomers with the desired $\alpha$ anomer being the major reaction product in both cases (Table 3 ). 
Table 3. Glycosylation in acetonitrile

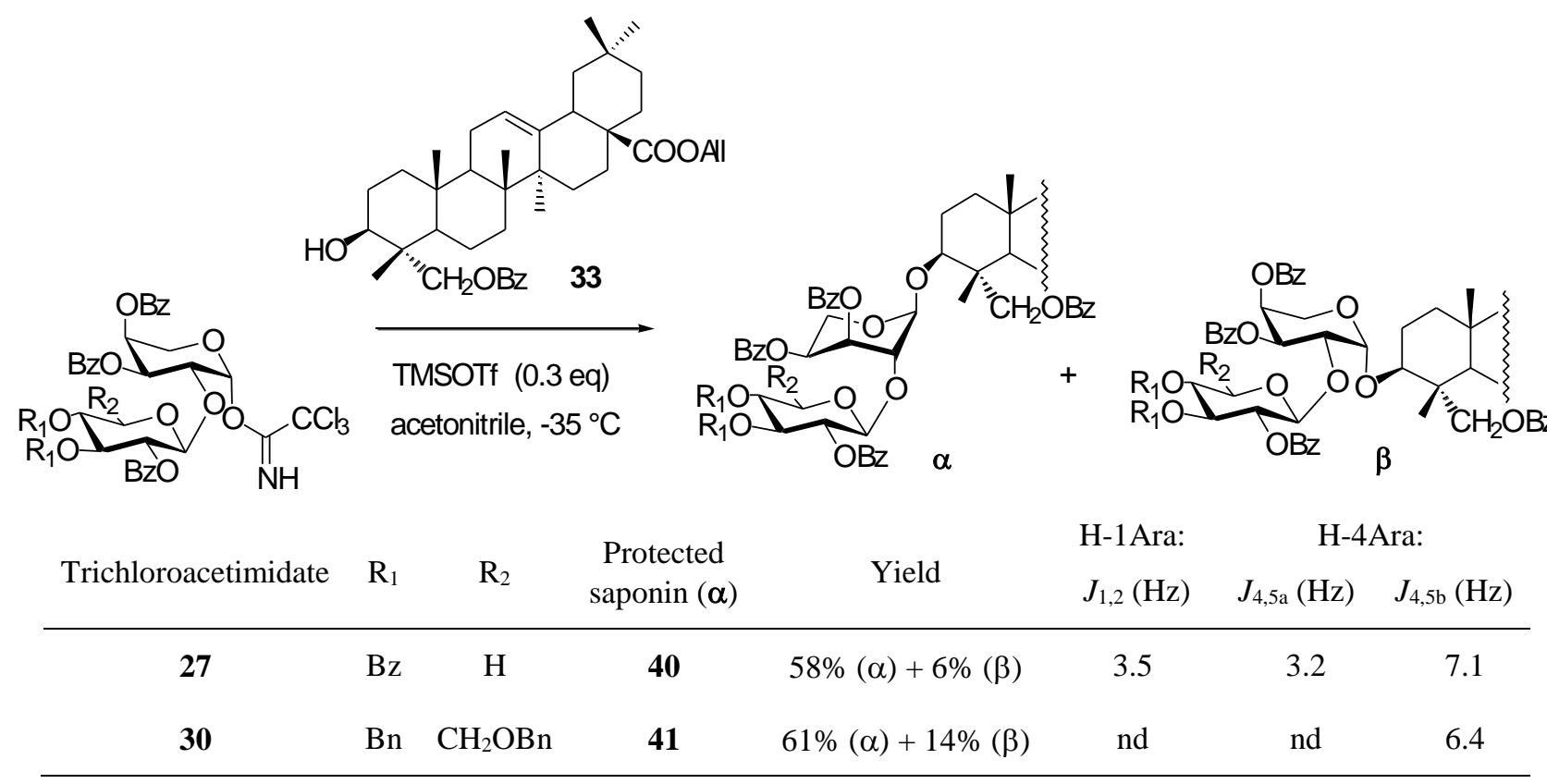

Separation of the two anomers was possible by reverse phase HPLC in 100\% acetonitrile. Based on the ${ }^{1} \mathrm{H}$ NMR coupling constants of the major $\alpha$ anomers $\left(J_{1,2}, J_{4,5 \mathrm{a}}\right.$ and $\left.J_{4,5 \mathrm{~b}}\right)$, it was observed that for compound 40 with a D-xylopyranosyl-L-arabinopyranose side chain the arabinopyranose ring adopts a ${ }^{1} C_{4}$ conformation to relieve steric hindrance. In the case of the saponin with a D-glucopyranose-Larabinopyranose side chain (41) the situation is not as clear-cut as several coupling constants remain undetermined.

Total deprotection of the saponin derivatives was performed in one or two steps based on the starting compound. While having previously reported the efficient removal of a hederagenin allyl ester in the presence of pyrrolidine and catalytic amounts of tetrakis(triphenylphosphine) palladium(0) $\left[\mathrm{Pd}\left(\mathrm{PPh}_{3}\right)_{4}\right]$, ${ }^{4,5}$ we sought to reduce the somewhat long reaction times necessary to achieve complete deprotection. In a normal de-allylation reaction, excess pyrrolidine serves as a nucleophile, driving the reaction to completion. ${ }^{24} \mathrm{~A}$ recent literature example describes the deprotection of allylphenols in a $10 \% \mathrm{KOH} / \mathrm{MeOH}$ solution in the presence of a catalytic amount of $\mathrm{Pd} / \mathrm{C}{ }^{25}$ We felt that replacing the pyrrolidine with an excess of $\mathrm{KOH}$ could lead to the deprotection of both the allyl and benzoyl protecting groups in one step. It was found that heating the saponin in the presence of 1 equivalent of $\mathrm{Pd}\left(\mathrm{PPh}_{3}\right)_{4}$ in a $3 \% \mathrm{KOH}$ solution in methanol at $60^{\circ} \mathrm{C}$ for 6 hours afforded the completely deprotected D-xylopyranosyl-L-arabinopyranose saponins (1-4) or the partially protected D-glucopyranosyl-L-arabinopyranose ones in good yield. For the latter compounds, the benzyl groups were then removed by hydrogenolysis in the presence of $\mathrm{Pd} / \mathrm{C}$ at atmospheric pressure (Table 4). Hydrogenolysis or migration of the double bond in the triterpenoid skeleton was not observed using these reaction conditions. Successful deprotection was also 
possible with a catalytic amount of $\mathrm{Pd}\left(\mathrm{PPh}_{3}\right)_{4}(0.3 \mathrm{eq})$ in a mixture of $\mathrm{THF} / 3 \% \mathrm{KOH} / \mathrm{MeOH}$ at 60 ${ }^{\circ} \mathrm{C}$, with the desired saponins being isolated in fair to excellent yields. The use of as little as $0.1 \mathrm{eq}$ of $\mathrm{Pd}\left(\mathrm{PPh}_{3}\right)_{4}$ was tried for the D-xylopyranose-L-arabinopyranose saponins, but in most cases the yields were poorer than those obtained with 0.3 eq of catalyst.

Table 4. Total deprotection of saponins: optimization of reaction conditions
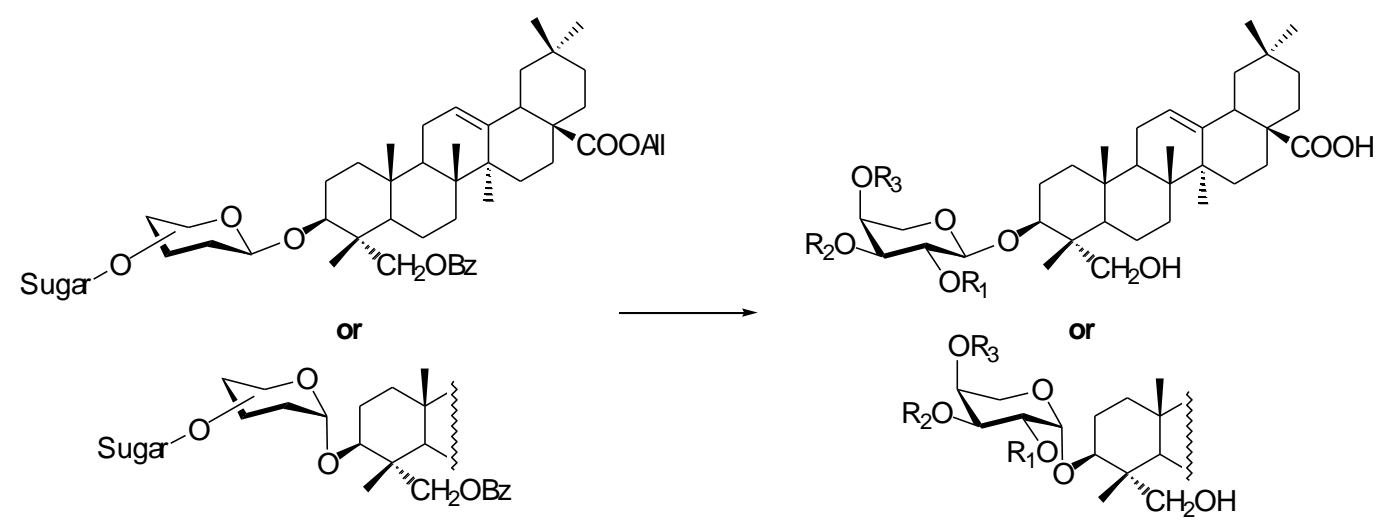

method A : $3 \% \mathrm{KOH} / \mathrm{MeOH}$ or THF/3\% KOH/MeOH, $\mathrm{Pd}\left(\mathrm{PPh}_{3}\right)_{4}, 60{ }^{\circ} \mathrm{C}$ method B : $1.3 \% \mathrm{KOH} / \mathrm{MeOH}$ or THF/3\% KOH/MeOH, $\mathrm{Pd}\left(\mathrm{PPh}_{3}\right)_{4}, 60{ }^{\circ} \mathrm{C} ; 2 . \mathrm{Pd} / \mathrm{C}, \mathrm{H}_{2}$

\begin{tabular}{|c|c|c|c|c|c|c|c|c|}
\hline $\begin{array}{l}\text { Protected } \\
\text { saponin }\end{array}$ & $\begin{array}{c}\text { Deprotection } \\
\text { Method }\end{array}$ & Saponin & $\mathrm{R}_{1}$ & $\mathrm{R}_{2}$ & $\mathrm{R}_{3}$ & $\begin{array}{c}\text { Yield } \\
(1 \text { eq Pd })^{c}\end{array}$ & $\begin{array}{c}\text { Yield } \\
(0.3 \text { eq Pd })^{d}\end{array}$ & $\begin{array}{c}\text { Yield } \\
(0.1 \text { eq Pd })^{d}\end{array}$ \\
\hline 40 & A & 1 & $\mathbf{X y l} \mathbf{l}^{\mathrm{a}}$ & $\mathrm{H}$ & $\mathrm{H}$ & $76 \%$ & $89 \%$ & $64 \%$ \\
\hline 38 & A & 2 & Xyl & $\mathrm{H}$ & $\mathrm{H}$ & $64 \%$ & $84 \%$ & $88 \%$ \\
\hline 34 & A & 3 & $\mathrm{H}$ & Xyl & $\mathrm{H}$ & $82 \%$ & $72 \%$ & $71 \%$ \\
\hline 35 & A & 4 & $\mathrm{H}$ & $\mathrm{H}$ & Xyl & $84 \%$ & $74 \%$ & $66 \%$ \\
\hline 41 & B & 5 & Glc $^{\mathrm{b}}$ & $\mathrm{H}$ & $\mathrm{H}$ & $82 \%$ & $87 \%$ & --- \\
\hline 39 & B & 6 & Glc & $\mathrm{H}$ & $\mathrm{H}$ & $88 \%$ & $82 \%$ & --- \\
\hline 36 & B & 7 & $\mathrm{H}$ & Glc & $\mathrm{H}$ & $78 \%$ & $84 \%$ & --- \\
\hline 37 & B & 8 & $\mathrm{H}$ & $\mathrm{H}$ & Glc & $82 \%$ & $65 \%$ & --- \\
\hline
\end{tabular}

${ }^{\mathrm{a}} \mathbf{X y \mathbf { l }}=(\beta$-D-xylopyranosyl $)$

${ }^{\mathrm{b}} \mathbf{G l c}=(\beta$-D-glucopyranosyl $)$

c $3 \% \mathrm{KOH} / \mathrm{MeOH}, 60{ }^{\circ} \mathrm{C}$

${ }^{\mathrm{d}} \mathrm{THF} / 3 \% \mathrm{KOH} / \mathrm{MeOH}, 60{ }^{\circ} \mathrm{C}$

The corresponding methyl esters (1a-8a) were then prepared in quantitative yield by diazomethane treatment of the acids $\mathbf{1 - 8}{ }^{26}$ 


\section{Conclusion}

Efficient chemical synthesis afforded a rapid access to eight hederagenin saponins, five of which are naturally occurring products whose synthesis has not yet been reported in the literature. The synthesis of six disaccharides consisting of an L-arabinopyranose substituted in positions 2,3 , or 4 with a $\beta$-D-xylopyranose or a $\beta$-D-glucopyranose residue was accomplished in good to excellent yields. Coupling of these disaccharides to a protected hederagenin derivative and total deprotection gave the desired saponins in 52\%-79\% overall yields. A deprotection method was developed with $\mathrm{Pd}\left(\mathrm{PPh}_{3}\right)_{4}$ in the presence of KOH to efficiently remove both the allyl ester and the sugar benzoyl protecting groups in one step. The fully deprotected saponins were thus obtained in good yields with significantly shorter reaction times.

The preparation of triterpenoid saponins in larger quantities facilitates the study of their biological activity. The strategy presented here opens the door to the synthesis of a wide variety of different saponins by simply changing the nature of the aglycone. In addition, structure-activity relationships can be more easily studied when all the positional isomers of a given sugar moiety are readily accessible. The hemolytic and cytotoxic activity of these molecules will be reported in due course.

\section{Experimental}

\subsection{General Methods}

All chemicals were reagent grade and used as supplied unless otherwise noted. Dichloromethane $\left(\mathrm{CH}_{2} \mathrm{Cl}_{2}\right)$ and triethylamine were refluxed over calcium hydride and distilled prior to use. All reactions were performed under an Argon atmosphere unless otherwise indicated. Analytical thinlayer chromatography (TLC) was performed on E. Merck Silica Gel $60 F_{254}$ plates. Compounds were visualized by dipping in an anisaldehyde solution in ethanol and heating. Column chromatography was performed using E. Merck Geduran Silica Gel Si 60 (40-60 $\mu \mathrm{M})$. Optical rotations were recorded at $22{ }^{\circ} \mathrm{C}$ with a Perkin-Elmer 241 polarimeter. ESI-MS were recorded with a Thermofinnigan quadripolar mass spectrometer with positive ion data collected automatically. High Resolution mass spectra were recorded on a Micromass Q-TOF spectrometer. NMR spectra were obtained using a Bruker Avance DRX 500 spectrometer $\left(500 \mathrm{MHz}\right.$ for ${ }^{1} \mathrm{H}$ and $125 \mathrm{MHz}$ for ${ }^{13} \mathrm{C}$ ). Elemental analyses were performed on a Perkin-Elmer CHN 2400. The HPLC system (Shimadzu) consisted of a solvent delivery system equipped with dual pumps (LC-8A), and a UV spectrophotometric detector (SPD-6A). Preparative HPLC was performed using a Merck 
Hibar column $(250 \mathrm{~mm} \times 25 \mathrm{~mm}$; Lichrospher RP $18(7 \mu \mathrm{m}))$. Protected saponins were detected at $230 \mathrm{~nm}$.

\subsection{4-Methoxybenzyl 2,3,4-tri- $O$-benzoyl- $\beta$-D-xylopyranosyl-(1 $\rightarrow 2)-3,4-d i-O$-benzoyl- $\alpha$-L-}

arabinopyranoside (13). General Method. In a typical experiment, 4-methoxybenzyl 3,4-di- $O$ benzoyl- $\alpha$-L-arabinopyranoside ${ }^{5} 9$ (1.5 g, 3.1 mmol), 2,3,4-tri- $O$-benzoyl- $\beta$-D-xylopyranosyl trichloroacetimidate $^{19} 12$ (2.85 g, $\left.4.7 \mathrm{mmol}, 1.5 \mathrm{eq}\right)$ and $4 \AA$ A powdered molecular sieves (6 g) were stirred for $1 \mathrm{~h}$ at room temperature in $\mathrm{CH}_{2} \mathrm{Cl}_{2}(75 \mathrm{~mL})$. The mixture was cooled to $-20{ }^{\circ} \mathrm{C}$ for 30 minutes followed by the dropwise addition of a $0.1 \mathrm{M}$ solution of TMSOTf in $\mathrm{CH}_{2} \mathrm{Cl}_{2}(1.55 \mathrm{~mL}$, $0.16 \mathrm{mmol}, 0.05 \mathrm{eq})$. After stirring for $2 \mathrm{~h}$ at this temperature, the reaction was quenched with triethylamine $(0.5 \mathrm{~mL})$, filtered through Celite and evaporated. The crude residue was purified by column chromatography (toluene/acetone 99:1 to 98:2) to give $2.62 \mathrm{~g}$ (90\%) of disaccharide 13 as an amorphous solid. $\mathrm{R}_{f}=0.47$ (toluene/acetone 9:1). $[\alpha]_{\mathrm{D}}=+32.9^{\circ}\left(c 1, \mathrm{CHCl}_{3}\right) .{ }^{1} \mathrm{H}^{\mathrm{NMR}}\left(\mathrm{CDCl}_{3}\right)$ : $\delta 3.70\left(\mathrm{dd}, 1 \mathrm{H}, J=12.7, J=5.3 \mathrm{~Hz}, \mathrm{H}-5\right.$ ') 3.85 (s, 3H, $\left.\mathrm{OCH}_{3}\right), 3.91(\mathrm{dd}, 1 \mathrm{H}, J=12.3, J=2.1 \mathrm{~Hz}$, H-5), 4.29 (dd, 1H, $J=12.4, J=4.7 \mathrm{~Hz}, \mathrm{H}-5), 4.39$ (dd, 1H, $J=7.3, J=5.5 \mathrm{~Hz}, \mathrm{H}-2), 4.57$ (dd, 1H, $J=12.8, J=3.7 \mathrm{~Hz}, \mathrm{H}-5^{\prime}$ ), 4.64 (d, 1H, $\left.J=10.9 \mathrm{~Hz}, \mathrm{CH}_{2} \mathrm{MPM}\right), 4.88$ (d, 1H, J=5.3 Hz, H-1), 4.98 (d, $\left.1 \mathrm{H}, J=10.9 \mathrm{~Hz}, \mathrm{CH}_{2} \mathrm{MPM}\right), 5.25$ (d, 1H, $J=4.2 \mathrm{~Hz}, \mathrm{H}-1$ '), 5.28 (m, 1H, H-4'), 5.35 (dd, 1H, $J$ $=6.2, J=4.3 \mathrm{~Hz}, \mathrm{H}-2$ ') $, 5.53(\mathrm{dd}, 1 \mathrm{H}, J=7.5, J=3.4 \mathrm{~Hz}, \mathrm{H}-3), 5.56$ (m, 1H, H-4), 5.71 (t, 1H, $J=$ $\left.6.3 \mathrm{~Hz}, \mathrm{H}-3^{\prime}\right), 6.91$ (d, 2H, $\left.J=8.6 \mathrm{~Hz}, \mathrm{Ar}-\mathrm{H}\right), 7.21$ (t, 1H, J= $\left.7.7 \mathrm{~Hz}, \mathrm{Ar}-\mathrm{H}\right), 7.27$ (t, 2H, $J=7.7$ $\mathrm{Hz}, \mathrm{Ar}-\mathrm{H}), 7.36-7.49$ (m, $11 \mathrm{H}, \mathrm{Ar}-\mathrm{H}), 7.59$ (m, 2H, Ar-H), 7.73 (d, 2H, J = 7.5 Hz, Ar-H), 7.86 (d, $2 \mathrm{H}, J=7.3 \mathrm{~Hz}, \mathrm{Ar}-\mathrm{H}), 7.99$ (d, 2H, $J=7.2 \mathrm{~Hz}, \mathrm{Ar}-\mathrm{H}), 8.05$ (d, 2H, J=7.0 Hz, Ar-H), 8.06 (d, 2H, $J=7.0 \mathrm{~Hz}, \mathrm{Ar}-\mathrm{H}) .{ }^{13} \mathrm{C} \mathrm{NMR}\left(\mathrm{CDCl}_{3}\right): \delta 55.3\left(\mathrm{OCH}_{3}\right), 60.8(\mathrm{C}-5), 61.5(\mathrm{C}-5), 68.0(\mathrm{C}-4), 68.7(\mathrm{C}-$ 4'), 69.2 (C-3'), 69.8 (C-2'), 70.7 (CH $2 \mathrm{MPM}), 71.8$ (C-3), 74.5 (C-2), 99.7 (C-1'), 100.1 (C-1), $113.8(\mathrm{CH}), 128.1(\mathrm{CH}), 128.3(\mathrm{CH}), 128.4(\mathrm{CH}), 128.4(\mathrm{CH}), 128.4(\mathrm{CH}), 128.8(\mathrm{C}), 129.0(\mathrm{C})$, $129.1(\mathrm{C}), 129.3(\mathrm{C}), 129.4(\mathrm{C}), 129.6(\mathrm{CH}), 129.7(\mathrm{CH}), 129.8(\mathrm{CH}), 129.9(\mathrm{CH}), 129.9(\mathrm{CH})$, 133.0 (C), 133.2 (C), 133.3 (C), 133.4 (C), 159.4 (C), 164.8 (CO), 165.1 (CO), 165.4 (CO), 165.4 (CO), 165.5 (CO). Anal. Calcd for $\mathrm{C}_{53} \mathrm{H}_{46} \mathrm{O}_{15}$ : C, 68.97; H, 5.02. Found: C, 68.60; H, 4.97.

\subsection{4-Methoxybenzyl 2,3,4-tri- $O$-benzoyl- $\beta$-D-xylopyranosyl-(1 $\rightarrow 3)$-2,4-di- $O$-benzoyl- $\alpha$-L-}

arabinopyranoside (14). This compound was prepared using the general method described for 13. Reaction of 4-methoxybenzyl 2,4-di- $O$-benzoyl- $\alpha$-L-arabinopyranoside $\mathbf{1 0}^{5}$ (0.5 g, $\left.1.0 \mathrm{mmol}\right)$ and trichloroacetimidate $12(0.95 \mathrm{~g}, 1.6 \mathrm{mmol})$ gave $0.68 \mathrm{~g}(70 \%)$ of $\mathbf{1 4}$. $\mathrm{R}_{f}=0.47$ (toluene/acetone 9:1). $[\alpha]_{\mathrm{D}}=+10.5^{\circ}\left(c 1, \mathrm{CHCl}_{3}\right) .{ }^{1} \mathrm{H} \mathrm{NMR}\left(\mathrm{CDCl}_{3}\right): \delta 3.67(\mathrm{dd}, 1 \mathrm{H}, J=12.4, J=5.8 \mathrm{~Hz}, \mathrm{H}-5$ '), 3.75 (dd, $1 \mathrm{H}, J=12.5, J=2.2 \mathrm{~Hz}, \mathrm{H}-5), 3.79$ (s, 3H, OCH $H_{3}, 4.32$ (dd, $\left.1 \mathrm{H}, J=7.8, J=3.3 \mathrm{~Hz}, \mathrm{H}-3\right), 4.35$ (m, 2H, H-5, H-5'), 4.52 (d, 1H, J=12.2 Hz, CH $2 \mathrm{MPM}), 4.63$ (d, 1H, J= 5.7 Hz, H-1), 4.67 (d, 1H, $J=$ 
$\left.12.2 \mathrm{~Hz}, \mathrm{CH}_{2} \mathrm{MPM}\right), 5.16$ (d, 1H, $J=4.7 \mathrm{~Hz}, \mathrm{H}-1^{\prime}$ ), 5.22 (m, 1H, H-4'), 5.34 (dd, 1H, $J=6.6, J=$ 5.0 Hz, H-2'), 5.56 (m, 1H, H-4), 5.62 (dd, 1H, J= 7.5, J=6.0 Hz, H-2), 5.65 (t, 1H, J=6.6 Hz, H3’), $6.71(\mathrm{~d}, 2 \mathrm{H}, J=8.5 \mathrm{~Hz}, \mathrm{Ar}-\mathrm{H}), 7.07$ (d, 2H, $J=8.5 \mathrm{~Hz}, \mathrm{Ar}-\mathrm{H}), 7.20-7.63$ (m, 15H, Ar-H), 7.81 (d, 2H, J=7.6 Hz, Ar-H), 7.95 (m, 4H, Ar-H), 8.01 (d, 2H, J = 7.5 Hz, Ar-H), 8.15 (d, 2H, J = 7.4 $\mathrm{Hz}, \mathrm{Ar}-\mathrm{H}) .{ }^{13} \mathrm{C} \mathrm{NMR}\left(\mathrm{CDCl}_{3}\right)$ : $\delta 55.1\left(\mathrm{OCH}_{3}\right), 60.9(\mathrm{C}-5$ '), 61.7 (C-5), $68.8(\mathrm{C}-4$ ') 69.3 (CH ${ }_{2}$ MPM), 69.7 (C-4, C-3'), 70.0 (C-2'), 70.8 (C-2), 76.5 (C-3), 98.4 (C-1), 100.4 (C-1'), 113.6 $(\mathrm{CH}), 128.1(\mathrm{CH}), 128.3(\mathrm{CH}), 128.3(\mathrm{CH}), 128.4(\mathrm{CH}), 128.8(\mathrm{C}), 129.0(\mathrm{C}), 129.2(\mathrm{C}), 129.3(\mathrm{C})$, $129.5(\mathrm{CH}), 129.7(\mathrm{CH}), 129.8(\mathrm{CH}), 129.8(\mathrm{CH}), 129.9(\mathrm{CH}), 132.9(\mathrm{CH}), 133.0(\mathrm{CH}), 133.2$ $(\mathrm{CH}), 159.2(\mathrm{C}), 164.7(\mathrm{CO}), 164.9(\mathrm{CO}), 165.2(\mathrm{CO}), 165.4(\mathrm{CO}), 166.1(\mathrm{CO})$. Anal. Calcd for $\mathrm{C}_{53} \mathrm{H}_{46} \mathrm{O}_{15}\left(\cdot 0,7 \mathrm{CH}_{3} \mathrm{OH}\right): \mathrm{C}, 68.23 ; \mathrm{H}, 5.20$. Found: C, 68.23; H, 5.11.

\subsection{4-Methoxybenzyl 2,3,4-tri- $O$-benzoyl- $\beta$-D-xylopyranosyl-(1 $\rightarrow$ 4)-2,3-di- $O$-benzoyl- $\alpha$-L-}

arabinopyranoside (15). This compound was prepared using the general method described for 13. Reaction of 4-methoxybenzyl 2,3-di- $O$-benzoyl- $\alpha$-L-arabinopyranoside $\mathbf{1 1}^{5}$ (0.5 g, $\left.1.0 \mathrm{mmol}\right)$ and trichloroacetimidate $12(0.95 \mathrm{~g}, 1.6 \mathrm{mmol})$ gave $0.89 \mathrm{~g}(92 \%)$ of $\mathbf{1 5}$. $\mathbf{R}_{f}=0.46$ (toluene/acetone 9:1).

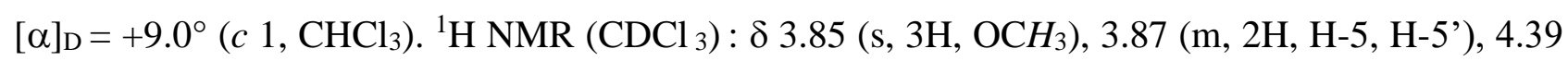
(dd, $1 \mathrm{H}, J=12.0, J=6.1 \mathrm{~Hz}, \mathrm{H}-5), 4.48$ (m, 1H, H-4), 4.57 (dd, 1H, $J=12.5, J=3.6 \mathrm{~Hz}, \mathrm{H}-5^{\prime}$ ), $4.64\left(\mathrm{~d}, 1 \mathrm{H}, J=11.5 \mathrm{~Hz}, \mathrm{CH} \mathrm{H}_{2} \mathrm{MPM}\right), 4.85$ (d, 1H, $\left.J=4.8 \mathrm{~Hz}, \mathrm{H}-1\right), 4.90$ (d, 1H, $J=11.5 \mathrm{~Hz}$, $\mathrm{CH}_{2} \mathrm{MPM}$ ), 5.14 (d, 1H, J=4.2 Hz, H-1'), 5.31 (m, 1H, H-4'), 5.42 (dd, 1H, J=6.0, J= 4.3 Hz, H2'), $5.53(\mathrm{dd}, 1 \mathrm{H}, J=7.1, J=3.2 \mathrm{~Hz}, \mathrm{H}-3), 5.66(\mathrm{dd}, 1 \mathrm{H}, J=7.0, J=4.9 \mathrm{~Hz}, \mathrm{H}-2), 5.75(\mathrm{t}, 1 \mathrm{H}, J=$ $\left.6.0 \mathrm{~Hz}, \mathrm{H}-3^{\prime}\right), 6.84$ (d, 2H, J=8.7 Hz, Ar-H), 7.14-7.62 (m, 17H, Ar-H), 7.82 (d, 2H, J = 8.3 Hz, Ar-H), $7.92(\mathrm{~d}, 2 \mathrm{H}, J=8.4 \mathrm{~Hz}, \mathrm{Ar}-\mathrm{H}), 8.02-8.07(\mathrm{~m}, 6 \mathrm{H}, \mathrm{Ar}-\mathrm{H}) .{ }^{13} \mathrm{C} \mathrm{NMR}\left(\mathrm{CDCl}_{3}\right): \delta 55.2$ $\left(\mathrm{OCH}_{3}\right), 60.5(\mathrm{C}-5$ ') $) 62.3$ (C-5), $68.4\left(\mathrm{C}^{2} 4^{\prime}\right), 68.8$ (C-3’), $69.5\left(\mathrm{C}-2^{\prime}\right), 69.8\left(\mathrm{CH}_{2} \mathrm{MPM}\right), 69.8(\mathrm{C}-2$, C-3), 72.1 (C-4), 98.1 (C-1), $99.4\left(\mathrm{C}-1^{\prime}\right), 113.7(\mathrm{CH}), 128.2(\mathrm{CH}), 128.4(\mathrm{CH}), 128.5(\mathrm{CH}), 128.9$ (C), $129.0(\mathrm{C}), 129.1(\mathrm{C}), 129.1(\mathrm{C}), 129.2(\mathrm{C}), 129.4(\mathrm{C}), 129.7(\mathrm{CH}), 129.8(\mathrm{CH}), 129.9(\mathrm{CH})$, $129.9(\mathrm{CH}), 133.0(\mathrm{CH}), 133.0(\mathrm{CH}), 133.2(\mathrm{CH}), 133.3(\mathrm{CH}), 133.3(\mathrm{CH}), 159.2(\mathrm{C}), 164.8(\mathrm{CO})$, 165.2 (CO), 165.5 (CO), 165.7 (CO). Anal. Calcd for $\mathrm{C}_{53} \mathrm{H}_{46} \mathrm{O}_{15}: \mathrm{C}, 68.97 ; \mathrm{H}, 5.02$. Found: C, $68.74 ; \mathrm{H}, 4.71$.

4.5. 3,4,6-Tri- $O$-acetyl-1,2- $O$-(1-ethylthioethylidene)- $\alpha$-D-glucopyranose (19). A solution of $\mathrm{HBr}$ in $\mathrm{AcOH}(33 \%, 38 \mathrm{~mL})$ was slowly added to a stirring solution of 1,2,3,4,6-penta- $O$-acetyl-Dglucopyranose $(10.5 \mathrm{~g}, 26.9 \mathrm{mmol})$ in $\mathrm{CH}_{2} \mathrm{Cl}_{2}(38 \mathrm{~mL})$ at $0{ }^{\circ} \mathrm{C}$. After stirring overnight at room temperature the reaction mixture was diluted with $\mathrm{CH}_{2} \mathrm{Cl}_{2}$, washed with $\mathrm{H}_{2} \mathrm{O}(200 \mathrm{~mL}), \mathrm{NaHCO}_{3}$ (sat.) $(2 \times 200 \mathrm{~mL}), \mathrm{NaCl}$ (sat.) and dried with $\mathrm{Na}_{2} \mathrm{SO}_{4}$. The solvent was evaporated and the residue $(11 \mathrm{~g})$ was taken up in nitromethane $(27 \mathrm{~mL})$. After addition of 2,6-lutidine $(4.7 \mathrm{~mL}, 40.4 \mathrm{mmol}$, 
$1.5 \mathrm{eq})$, ethanethiol $(8.0 \mathrm{~mL}, 107.6 \mathrm{mmol}, 4 \mathrm{eq})$ and tetrabutylammonium bromide $(0.87 \mathrm{~g}, 2.7$ mmol, $0.1 \mathrm{eq}$ ), the reaction was stirred at room temperature for $48 \mathrm{~h}$. The solution was then partitioned between EtOAc and aq. $\mathrm{NaHCO}_{3}$. The aqueous layer was extracted with EtOAc, dried $\left(\mathrm{Na}_{2} \mathrm{SO}_{4}\right)$, filtered and evaporated. The residue was purified by column chromatography (cyclohexane/EtOAc 8:2 to 7:3) to give $9.2 \mathrm{~g}$ (83\%) of $\mathbf{1 9}$ as an oil. ${ }^{1} \mathrm{H}$ and ${ }^{13} \mathrm{C}$ NMR spectra were performed in deuterated chloroform and were in accordance with published data. ${ }^{27}$

4.6. 3,4,6-Tri- $O$-benzyl-1,2- $O$-(1-ethylthioethylidene)- $\alpha$-D-glucopyranose (20). To a solution of orthoester 19 (8.9 g, $22.7 \mathrm{mmol})$ and benzyl bromide ( $8.7 \mathrm{~mL}, 72.6 \mathrm{mmol}, 3.2 \mathrm{eq})$ in dry THF (55 $\mathrm{mL}$ ) was added powdered $\mathrm{KOH}(14 \mathrm{~g}, 250 \mathrm{mmol}, 11 \mathrm{eq})$ and the reaction was refluxed overnight with stirring. After the mixture was cooled, EtOAc was added, and the solution was successively washed with $\mathrm{H}_{2} \mathrm{O}(3 \times), \mathrm{NaHCO}_{3}$ (sat) (2x), and $\mathrm{H}_{2} \mathrm{O}(2 \times)$. The organic layer was dried $\left(\mathrm{Na}_{2} \mathrm{SO}_{4}\right)$, evaporated, and the crude residue was purified by column chromatography (cyclohexane/EtOAc 97:3) to give $6.8 \mathrm{~g}(55 \%)$ of $\mathbf{2 0}$ as an oil. $[\alpha]_{\mathrm{D}}=+17.1^{\circ}\left(c 1, \mathrm{CHCl}_{3}\right) .{ }^{1} \mathrm{H} \mathrm{NMR}\left(\mathrm{CDCl}_{3}\right): \delta 1.34(\mathrm{t}$, $3 \mathrm{H}, J=7.5 \mathrm{~Hz}, \mathrm{SCH}_{2} \mathrm{CH}_{3}$ ), 1.99 (s, 3H, $\mathrm{CH}_{3}$ orthoester), 2.69 (q, $2 \mathrm{H}, J=7.5 \mathrm{~Hz}, \mathrm{SCH}_{2} \mathrm{CH}_{3}$ ), 3.69 (dd, 1H, $J=10.9, J=4.2 \mathrm{~Hz}, \mathrm{H}-6 \mathrm{a}), 3.73$ (dd, 1H, $J=10.9, J=1.9 \mathrm{~Hz}, \mathrm{H}-6 \mathrm{~b}), 3.79$ (dd, 1H, $J=$ 9.5, $J=3.1 \mathrm{~Hz}, \mathrm{H}-4), 3.89$ (m, 1H, H-5), 3.99 (t, 1H, $J=3.0 \mathrm{~Hz}, \mathrm{H}-3), 4.41$ (d, 1H, $J=11.5 \mathrm{~Hz}$, $\left.\mathrm{CH}_{2} \mathrm{Ph}\right), 4.58\left(\mathrm{~d}, 1 \mathrm{H}, J=12.2 \mathrm{~Hz}, \mathrm{CH}_{2} \mathrm{Ph}\right), 4.60\left(\mathrm{~d}, 1 \mathrm{H}, J=11.5 \mathrm{~Hz}, \mathrm{CH}_{2} \mathrm{Ph}\right), 4.63(\mathrm{~m}, 1 \mathrm{H}, \mathrm{H}-2)$, $4.64\left(\mathrm{~d}, 1 \mathrm{H}, J=11.9 \mathrm{~Hz}, \mathrm{CH}_{2} \mathrm{Ph}\right), 4.65$ (d, 1H, $\left.J=11.2 \mathrm{~Hz}, \mathrm{CH}_{2} \mathrm{Ph}\right), 4.75$ (d, 1H, $J=11.9 \mathrm{~Hz}$, $\left.\mathrm{CH}_{2} \mathrm{Ph}\right), 5.85(\mathrm{~d}, 1 \mathrm{H}, J=5.3 \mathrm{~Hz}, \mathrm{H}-1), 7.24-7.43(\mathrm{~m}, 15 \mathrm{H}, \mathrm{Ar}-\mathrm{H}) .{ }^{13} \mathrm{C} \mathrm{NMR}\left(\mathrm{CDCl}_{3}\right): \delta 15.2$ $\left(\mathrm{SCH}_{2} \mathrm{CH}_{3}\right), 24.8\left(\mathrm{SCH}_{2} \mathrm{CH}_{3}\right), 27.8\left(\mathrm{CH}_{3}\right.$ orthoester $), 69.1$ (C-6), $70.1(\mathrm{C}-5), 71.7\left(\mathrm{CH}_{2} \mathrm{Ph}\right), 72.5$ $\left(\mathrm{CH}_{2} \mathrm{Ph}\right), 73.4\left(\mathrm{CH}_{2} \mathrm{Ph}\right), 74.5(\mathrm{C}-2), 75.1(\mathrm{C}-4), 77.4$ (C-3), $98.2(\mathrm{C}-1), 115.7$ (C orthoester), 127.6 $(\mathrm{CH}), 127.8(\mathrm{CH}), 127.9(\mathrm{CH}), 128.0(\mathrm{CH}), 128.3(\mathrm{CH}), 128.4(\mathrm{CH}), 128.5(\mathrm{CH}), 137.6(\mathrm{C}), 137.8$ (C), 138.1 (C). Anal. Calcd for $\mathrm{C}_{31} \mathrm{H}_{36} \mathrm{O}_{6} \mathrm{~S}$ : C, 69.38; H, 6.76. Found: C, 69.50; H, 6.88.

4.7. Ethyl 2-O-acetyl-3,4,6-tri- $O$-benzyl-1-thio- $\beta$-D-glucopyranoside (21). To a solution of orthoester 20 (6.6 g, $12.3 \mathrm{mmol})$ in $\mathrm{CH}_{2} \mathrm{Cl}_{2}(26 \mathrm{~mL})$ was added $4 \AA$ molecular sieves $(1.6 \mathrm{~g})$, and the mixture was stirred for $1 \mathrm{~h}$. The solution was cooled to $0{ }^{\circ} \mathrm{C}$, and TMSOTf $(0.11 \mathrm{~mL}$, mmol, $0.05 \mathrm{eq}$ ) was slowly added. After stirring for $4 \mathrm{~h}$, the reaction was quenched by the addition of $\mathrm{Et}_{3} \mathrm{~N}$, filtered through celite and evaporated to dryness to give $6.4 \mathrm{~g}$ of a crude product which was used without further purification in the next step. For identification purposes, a small amount of product was purified by column chromatography (cyclohexane/EtOAc 95:5). $[\alpha]_{\mathrm{D}}=+8.6^{\circ}\left(\mathrm{c} 1, \mathrm{CHCl}_{3}\right) .{ }^{1} \mathrm{H}$ $\mathrm{NMR}\left(\mathrm{CDCl}_{3}\right): \delta 1.33\left(\mathrm{t}, 3 \mathrm{H}, J=7.4 \mathrm{~Hz}, \mathrm{SCH}_{2} \mathrm{CH}_{3}\right), 2.05\left(\mathrm{~s}, 3 \mathrm{H}, \mathrm{CH} \mathrm{H}_{3} \mathrm{CO}\right), 2.78(\mathrm{~m}, 2 \mathrm{H}$, $\left.\mathrm{SCH}_{2} \mathrm{CH}_{3}\right), 3.57$ (m, 1H, H-5), 3.74 (t, $\left.1 \mathrm{H}, J=9.0 \mathrm{~Hz}, \mathrm{H}-3\right), 3.77$ (t, $\left.1 \mathrm{H}, J=8.8 \mathrm{~Hz}, \mathrm{H}-4\right), 3.78$ (dd, $1 \mathrm{H}, J=11.3, J=4.5 \mathrm{~Hz}, \mathrm{H}-6 \mathrm{a}), 3.83$ (dd, 1H, $J=11.1, J=1.9 \mathrm{~Hz}, \mathrm{H}-6 \mathrm{~b}), 4.43$ (d, 1H, $J=10.0 \mathrm{~Hz}$, 
$\mathrm{H}-1), 4.62\left(\mathrm{~d}, 1 \mathrm{H}, J=12.2 \mathrm{~Hz}, \mathrm{CH}_{2} \mathrm{Ph}\right), 4.64\left(\mathrm{~d}, 1 \mathrm{H}, J=11.0 \mathrm{~Hz}, \mathrm{CH}_{2} \mathrm{Ph}\right), 4.68(\mathrm{~d}, 1 \mathrm{H}, J=12.1 \mathrm{~Hz}$, $\left.\mathrm{CH}_{2} \mathrm{Ph}\right), 4.76\left(\mathrm{~d}, 1 \mathrm{H}, J=11.4 \mathrm{~Hz}, \mathrm{CH}_{2} \mathrm{Ph}\right), 4.86\left(\mathrm{~d}, 1 \mathrm{H}, J=10.6 \mathrm{~Hz}, \mathrm{CH}_{2} \mathrm{Ph}\right), 4.88(\mathrm{~d}, 1 \mathrm{H}, J=11.3$ $\left.\mathrm{Hz}, \mathrm{CH}_{2} \mathrm{Ph}\right), 5.10(\mathrm{t}, 1 \mathrm{H}, J=9.2 \mathrm{~Hz}, \mathrm{H}-2), 7.26-7.41(\mathrm{~m}, 15 \mathrm{H}, \mathrm{Ar}-\mathrm{H}) .{ }^{13} \mathrm{C} \mathrm{NMR}\left(\mathrm{CDCl}_{3}\right): \delta 14.9$ $\left(\mathrm{SCH}_{2} \mathrm{CH}_{3}\right), 21.0\left(\mathrm{CH}_{3} \mathrm{CO}\right), 23.8\left(\mathrm{SCH}_{2} \mathrm{CH}_{3}\right), 68.8(\mathrm{C}-6), 71.7(\mathrm{C}-2), 73.4\left(\mathrm{CH}_{2} \mathrm{Ph}\right), 75.1\left(\mathrm{CH}_{2} \mathrm{Ph}\right)$, $75.2\left(\mathrm{CH}_{2} \mathrm{Ph}\right), 77.8(\mathrm{C}-4), 79.4(\mathrm{C}-5), 83.4(\mathrm{C}-1), 84.4(\mathrm{C}-3), 127.6(\mathrm{CH}), 127.8(\mathrm{CH}), 127.9(\mathrm{CH})$, $128.0(\mathrm{CH}), 128.3(\mathrm{CH}), 128.4(\mathrm{CH}), 137.9(\mathrm{C}), 138.1(\mathrm{C}), 138.2(\mathrm{C}), 169.6(\mathrm{CO})$. Anal. Calcd for $\mathrm{C}_{31} \mathrm{H}_{36} \mathrm{O}_{6} \mathrm{~S}: \mathrm{C}, 69.38 ; \mathrm{H}, 6.76$. Found: C, 69.23; H, 6.88.

4.8. Ethyl 2- $\boldsymbol{O}$-benzoyl-3,4,6-tri- $\boldsymbol{O}$-benzyl-1-thio- $\boldsymbol{\beta}$-D-glucopyranoside (22). The crude acetate $21(6.3 \mathrm{~g})$ was dissolved in a solution of $3 \% \mathrm{KOH} / \mathrm{MeOH}(80 \mathrm{~mL})$ and was stirred overnight. The reaction mixture was then diluted with EtOAc, washed with $\mathrm{H}_{2} \mathrm{O}(2 \times)$, dried $\left(\mathrm{Na}_{2} \mathrm{SO}_{4}\right)$, filtered and evaporated. The crude product was taken up in pyridine $(35 \mathrm{~mL})$ and the reaction was cooled to 0 ${ }^{\circ} \mathrm{C}$. Benzoyl chloride $(2.8 \mathrm{~mL}, 24.6 \mathrm{mmol}, 2 \mathrm{eq})$ was added dropwise, and after warming to room temperature, the reaction was heated to $70{ }^{\circ} \mathrm{C}$ for $6 \mathrm{~h}$. The solvent was then removed under reduced pressure and the residue dissolved in EtOAc. The organic layer was washed with $\mathrm{H}_{2} \mathrm{O}, 1 \mathrm{~N} \mathrm{HCl}$, $\mathrm{NaHCO}_{3}$ (sat.), and dried $\left(\mathrm{Na}_{2} \mathrm{SO}_{4}\right)$. After filtration and evaporation of the solvent under reduced pressure the crude product was purified by column chromatography (cyclohexane/EtOAc 92:8) to give $3.45 \mathrm{~g}(49 \%)$ of benzoate 22 as an amorphous solid. $[\alpha]_{\mathrm{D}}=+28.3^{\circ}\left(c 1, \mathrm{CHCl}_{3}\right) .{ }^{1} \mathrm{H}$ NMR $\left(\mathrm{CDCl}_{3}\right): \delta 1.31\left(\mathrm{t}, 3 \mathrm{H}, J=7.4, \mathrm{SCH}_{2} \mathrm{CH}_{3}\right), 2.79\left(\mathrm{~m}, 2 \mathrm{H}, \mathrm{SCH}_{2} \mathrm{CH}_{3}\right), 3.64(\mathrm{~m}, 1 \mathrm{H}, \mathrm{H}-5), 3.81$ (dd, $1 \mathrm{H}, J=11.0, J=4.7, \mathrm{H}-6 \mathrm{a}), 3.83$ (t, 1H, $J=8.8, \mathrm{H}-4), 3.86$ (dd, 1H, $J=10.8, J=1.7, \mathrm{H}-6 \mathrm{~b}), 3.91$ (t, $1 \mathrm{H}, J=9.0, \mathrm{H}-3), 4.60$ (d, 1H, $J=10.0, \mathrm{H}-1), 4.64$ (d, 1H, $\left.J=12.1, \mathrm{CH}_{2} \mathrm{Ph}\right), 4.67$ (d, 1H, $J=11.5$, $\left.\mathrm{CH}_{2} \mathrm{Ph}\right), 4.69\left(\mathrm{~d}, 1 \mathrm{H}, J=12.5, \mathrm{CH}_{2} \mathrm{Ph}\right), 4.73\left(\mathrm{~d}, 1 \mathrm{H}, J=11.1, \mathrm{CH}_{2} \mathrm{Ph}\right), 4.81(\mathrm{~d}, 1 \mathrm{H}, J=11.1$, $\left.\mathrm{CH}_{2} \mathrm{Ph}\right), 4.89\left(\mathrm{~d}, 1 \mathrm{H}, J=10.9, \mathrm{CH}_{2} \mathrm{Ph}\right), 5.38(\mathrm{t}, 1 \mathrm{H}, J=9.6, \mathrm{H}-2), 7.26-8.10(\mathrm{~m}, 20 \mathrm{H}, \mathrm{Ar}-\mathrm{H}) .{ }^{13} \mathrm{C}$ NMR $\left(\mathrm{CDCl}_{3}\right): \delta 14.9\left(\mathrm{SCH}_{2} \mathrm{CH}_{3}\right), 23.8\left(\mathrm{SCH}_{2} \mathrm{CH}_{3}\right), 68.9(\mathrm{C}-6), 72.4(\mathrm{C}-2), 73.5\left(\mathrm{CH}_{2} \mathrm{Ph}\right), 75.1$ $\left(\mathrm{CH}_{2} \mathrm{Ph}\right), 75.3\left(\mathrm{CH}_{2} \mathrm{Ph}\right), 77.9(\mathrm{C}-4), 79.5(\mathrm{C}-5), 83.4(\mathrm{C}-1), 84.3(\mathrm{C}-3), 127.6(\mathrm{CH}), 127.7(\mathrm{CH})$, $127.8(\mathrm{CH}), 128.0(\mathrm{CH}), 128.2(\mathrm{CH}), 128.4(\mathrm{CH}), 129.8(\mathrm{CH}), 129.9(\mathrm{C}), 133.1(\mathrm{CH}), 137.7(\mathrm{C})$, 137.9 (C), 138.1 (C), 165.3 (CO). Anal. Calcd for $\mathrm{C}_{36} \mathrm{H}_{38} \mathrm{O}_{6} \mathrm{~S}: \mathrm{C}, 72.02$; H, 6.4. Found: C, 72.02; H, 6.31 .

4.9. 4-Methoxybenzyl 2-O-benzoyl-3,4,6-tri- $O$-benzyl- $\beta$-D-glucopyranosyl-(1 $\rightarrow 2)-3,4-d i-O$ benzoyl- $\alpha$-L-arabinopyranoside (23). General Method. In a typical experiment, a mixture of alcohol 9 (3.0 g, $6.3 \mathrm{mmol})$, thioglycoside 22 (5.63 g, $9.4 \mathrm{mmol}, 1.5 \mathrm{eq})$, and $4 \AA$ powdered molecular sieves (15 g) was stirred for $2 \mathrm{~h}$ at room temperature in $\mathrm{CH}_{2} \mathrm{Cl}_{2}(72 \mathrm{~mL})$. The mixture was cooled to $0{ }^{\circ} \mathrm{C}$ and $\mathrm{N}$-iodosuccinimide $(2.12 \mathrm{~g}, 9.4 \mathrm{mmol}, 1.5 \mathrm{eq})$ was added followed by the dropwise addition of triflic acid $(0.028 \mathrm{~mL}, 0.05 \mathrm{eq})$. After $2 \mathrm{~h}$ at $0^{\circ} \mathrm{C}$, the reaction was quenched 
with triethylamine and filtered through Celite. The filtrate was washed with $\mathrm{NaHCO}_{3}, 10 \%$ $\mathrm{Na}_{2} \mathrm{~S}_{2} \mathrm{O}_{3}$, and water. The organic layer was dried $\left(\mathrm{Na}_{2} \mathrm{SO}_{4}\right)$, filtered and evaporated. The crude residue was purified by column chromatography (toluene/acetone 99:1) to give $5.22 \mathrm{~g}(82 \%)$ of disaccharide 23 as a white amorphous solid. $\mathrm{R}_{f}=0.58$ (toluene/acetone 9:1). $[\alpha]_{\mathrm{D}}=+33.4^{\circ}$ (c 1 , $\mathrm{CHCl}_{3}$ ). ${ }^{1} \mathrm{H} \mathrm{NMR}_{\left(\mathrm{CDCl}_{3}\right)}$ : $\delta 3.63$ (m, 1H, H-5'), 3.82 (m, 4H, H-6'a/b, H-3', H-5a), 3.84 (s, 3H, $\mathrm{OCH}_{3}$ ), 3.86 (t, 1H, J=9.5 Hz, H-4'), 4.17 (dd, 1H, $\left.J=11.3, J=8.0 \mathrm{~Hz}, \mathrm{H}-5 \mathrm{~b}\right), 4.26$ (m, 1H, H-2), $4.54\left(\mathrm{~d}, 1 \mathrm{H}, J=11.1 \mathrm{~Hz}, \mathrm{CH}_{2} \mathrm{MPM}\right), 4.61\left(\mathrm{~d}, 1 \mathrm{H}, J=12.1 \mathrm{~Hz}, \mathrm{CH}_{2} \mathrm{Ph}\right), 4.64$ (m, 3H, $\left.\mathrm{CH}_{2} \mathrm{Ph}\right), 4.75$ (d, $\left.1 \mathrm{H}, J=11.0 \mathrm{~Hz}, \mathrm{CH}_{2} \mathrm{Ph}\right), 4.83$ (d, $\left.1 \mathrm{H}, J=11.1 \mathrm{~Hz}, \mathrm{CH}_{2} \mathrm{MPM}\right), 4.87$ (d, $1 \mathrm{H}, J=10.9 \mathrm{~Hz}$, $\left.\mathrm{CH}_{2} \mathrm{Ph}\right), 4.90(\mathrm{~d}, 1 \mathrm{H}, J=7.9 \mathrm{~Hz}, \mathrm{H}-1$ '), 4.96 (d, 1H, $J=2.6 \mathrm{~Hz}, \mathrm{H}-1), 5.32$ (m, 1H, H-4), 5.38 (t, $1 \mathrm{H}, J=8.3 \mathrm{~Hz}, \mathrm{H}-2^{\prime}$ ), 5.46 (m, 1H, H-3), 6.87 (d, 2H, J=8.2 Hz, Ar-H), 7.26-7.67 (m, 26H, Ar-H), 7.85 (d, 2H, $J=7.9 \mathrm{~Hz}, \mathrm{Ar}-\mathrm{H}), 7.90$ (d, 2H, J=7.9 Hz, Ar-H), 7.96 (d, 2H, J=7.8 Hz, Ar-H). ${ }^{13} \mathrm{C}$

NMR $\left(\mathrm{CDCl}_{3}\right): \delta 55.3\left(\mathrm{OCH}_{3}\right), 58.8(\mathrm{C}-5), 66.8(\mathrm{C}-4), 68.7$ (C-6’), 69.7 (C-3), $69.8\left(\mathrm{CH}_{2} \mathrm{MPM}\right)$, 73.4 (C-2'), $73.6\left(\mathrm{CH}_{2} \mathrm{Ph}\right), 74.9$ (C-2), 75.0 (2 × $\left.\mathrm{CH}_{2} \mathrm{Ph}\right), 75.5$ (C-5'), 77.8 (C-4'), 82.6 (C-3'), 98.9 (C-1), $100.9\left(\mathrm{C}-1^{\prime}\right), 113.7(\mathrm{CH}), 127.6(\mathrm{CH}), 127.6(\mathrm{CH}), 127.8(\mathrm{CH}), 127.8(\mathrm{CH}), 128.0(\mathrm{CH})$, $128.0(\mathrm{CH}), 128.3(\mathrm{CH}), 128.3(\mathrm{CH}), 128.4(\mathrm{CH}), 129.5(\mathrm{C}), 129.6(\mathrm{C}), 129.7(\mathrm{CH}), 129.7(\mathrm{CH})$, $132.9(\mathrm{CH}), 133.0(\mathrm{CH}), 133.1(\mathrm{CH}), 137.6(\mathrm{C}), 137.9(\mathrm{C}), 138.2(\mathrm{C}), 159.4(\mathrm{C}), 164.9(\mathrm{CO}), 165.1$ (CO), 165.6 (CO). Anal. Calcd for $\mathrm{C}_{61} \mathrm{H}_{58} \mathrm{O}_{14}$ : C, 72.18; H, 5.76. Found: C, 71.91; H, 5.89.

\subsection{4-Methoxybenzyl 2-O-benzoyl-3,4,6-tri- $O$-benzyl- $\beta$-D-glucopyranosyl-(1 $\rightarrow 3)-2,4-d i-O$ -}

benzoyl- $\alpha$-L-arabinopyranoside (24). This compound was prepared using the general method described for 23. Reaction of 4-methoxybenzyl 2,4-di- $O$-benzoyl- $\alpha$-L-arabinopyranoside $\mathbf{1 0}^{5}(0.96$ $\mathrm{g}, 2.0 \mathrm{mmol})$ and thioglycoside $22(1.8 \mathrm{~g}, 3.0 \mathrm{mmol})$ gave $1.06 \mathrm{~g}(52 \%)$ of $\mathbf{2 4}$. $\mathrm{R}_{f}=0.52$ (toluene/acetone 9:1). $[\alpha]_{\mathrm{D}}=+45.4^{\circ}\left(c 1, \mathrm{CHCl}_{3}\right) .{ }^{1} \mathrm{H}_{\mathrm{NMR}}\left(\mathrm{CDCl}_{3}\right): \delta 3.57(\mathrm{~m}, 1 \mathrm{H}, \mathrm{H}-5$ '), 3.64 (dd, 1H, $J=12.6, J=2.2 \mathrm{~Hz}, \mathrm{H}-5 \mathrm{a}), 3.66$ (m, 2H, H-6'a/b), 3.73 (t, 1H, J=8.8 Hz, H-4'), 3.76 (t, $1 \mathrm{H}, J=8.7 \mathrm{~Hz}, \mathrm{H}-3$ ') 3.79 (s, 3H, $\left.\mathrm{OCH}_{3}\right), 4.24$ (dd, 1H, $\left.J=7.9, J=3.5 \mathrm{~Hz}, \mathrm{H}-3\right), 4.31$ (dd, 1H, $J=$ 12.6, $J=4.7 \mathrm{~Hz}, \mathrm{H}-5 \mathrm{~b}), 4.39$ (d, $\left.1 \mathrm{H}, J=12.0 \mathrm{~Hz}, \mathrm{CH}_{2} \mathrm{Ph}\right), 4.42$ (d, $\left.1 \mathrm{H}, J=11.9 \mathrm{~Hz}, \mathrm{CH}_{2} \mathrm{Ph}\right), 4.45$ (d, $\left.1 \mathrm{H}, J=12.4 \mathrm{~Hz}, \mathrm{CH}_{2} \mathrm{MPM}\right), 4.50$ (d, $\left.1 \mathrm{H}, J=5.9 \mathrm{~Hz}, \mathrm{H}-1\right), 4.55$ (m, 2H, $\mathrm{CH}_{2} \mathrm{Ph}, \mathrm{CH}_{2} \mathrm{MPM}$ ), $4.58\left(\mathrm{~d}, 1 \mathrm{H}, J=11.1 \mathrm{~Hz}, \mathrm{CH}_{2} \mathrm{Ph}\right), 4.66\left(\mathrm{~d}, 1 \mathrm{H}, J=11.1 \mathrm{~Hz}, \mathrm{CH}_{2} \mathrm{Ph}\right), 4.77$ (d, 1H, $J=10.9 \mathrm{~Hz}$, $\left.\mathrm{CH}_{2} \mathrm{Ph}\right), 4.80$ (d, 1H, J= 7.7 Hz, H-1'), 5.28 (t, 1H, J= $8.2 \mathrm{~Hz}, \mathrm{H}-2$ '), 5.50 (m, 2H, H-2, H-4), 6.69 $(\mathrm{d}, 2 \mathrm{H}, J=8.7 \mathrm{~Hz}, \mathrm{Ar}-\mathrm{H}), 6.97$ (d, 2H, $J=8.6 \mathrm{~Hz}, \mathrm{Ar}-\mathrm{H}), 7.07-7.60$ (m, 24H, Ar-H), 7.83 (d, 2H, $J$ $=7.6 \mathrm{~Hz}, \mathrm{Ar}-\mathrm{H}), 7.86(\mathrm{~d}, 2 \mathrm{H}, J=7.3 \mathrm{~Hz}, \mathrm{Ar}-\mathrm{H}), 8.16(\mathrm{dd}, 2 \mathrm{H}, J=8.4, J=1.2 \mathrm{~Hz}, \mathrm{Ar}-\mathrm{H}) .{ }^{13} \mathrm{C} \mathrm{NMR}$ $\left(\mathrm{CDCl}_{3}\right): \delta 55.1\left(\mathrm{OCH}_{3}\right), 61.9(\mathrm{C}-5), 68.9\left(\mathrm{CH}_{2} \mathrm{MPM}\right), 70.0(\mathrm{C}-4), 71.2(\mathrm{C}-2), 73.4\left(\mathrm{CH}_{2} \mathrm{Ph}\right), 73.4$ (C-2'), $74.8\left(\mathrm{CH}_{2} \mathrm{Ph}\right), 74.9\left(\mathrm{CH}_{2} \mathrm{Ph}\right), 75.2\left(\mathrm{C}^{\prime} 5^{\prime}, \mathrm{C}-3\right), 77.7\left(\mathrm{C}^{\prime} 4^{\prime}\right), 82.7\left(\mathrm{C}^{\prime} 3^{\prime}\right), 98.1(\mathrm{C}-1), 101.1$ $\left(\mathrm{C}^{\prime} 1^{\prime}\right), 113.6(\mathrm{CH}), 127.5(\mathrm{CH}), 127.6(\mathrm{CH}), 127.8(\mathrm{CH}), 127.8(\mathrm{CH}), 128.0(\mathrm{CH}), 128.1(\mathrm{CH})$, $128.2(\mathrm{CH}), 128.3(\mathrm{CH}), 128.3(\mathrm{CH}), 128.4(\mathrm{CH}), 128.8(\mathrm{C}), 129.6(\mathrm{CH}), 129.7(\mathrm{CH}), 129.8(\mathrm{C})$, 
$130.1(\mathrm{CH}), 132.6(\mathrm{CH}), 133.0(\mathrm{CH}), 137.7(\mathrm{C}), 137.9(\mathrm{C}), 138.2(\mathrm{C}), 159.1(\mathrm{C}), 164.7(\mathrm{CO}), 166.3$ (CO). Anal. Calcd for $\mathrm{C}_{61} \mathrm{H}_{58} \mathrm{O}_{14}$ : C, 72.18; H, 5.76. Found: C, 71.95; H, 5.86.

\subsection{4-Methoxybenzyl 2-O-benzoyl-3,4,6-tri- $O$-benzyl- $\beta$-D-glucopyranosyl-(1 $\rightarrow$ 4)-2,3-di- $O$ -} benzoyl- $\alpha$-L-arabinopyranoside (25). This compound was prepared using the general method

described for 23. Reaction of 4-methoxybenzyl 2,3-di-O-benzoyl- $\alpha$-L-arabinopyranoside $\mathbf{1 1}^{5}(0.5$ $\mathrm{g}, 1.0 \mathrm{mmol})$ and thioglycoside $22(0.94 \mathrm{~g}, 1.6 \mathrm{mmol})$ gave $0.87 \mathrm{~g}(82 \%)$ of $\mathbf{2 5}$. $\mathrm{R}_{f}=0.56$ (toluene/acetone 9:1). $[\alpha]_{\mathrm{D}}=+7.2^{\circ}\left(c 1, \mathrm{CHCl}_{3}\right) .{ }^{1} \mathrm{H} \mathrm{NMR}\left(\mathrm{CDCl}_{3}\right): \delta 3.61\left(\mathrm{~m}, 1 \mathrm{H}, \mathrm{H}-5^{\prime}\right), 3.77(\mathrm{~m}$, 2H, H-6'a/b), 3.82 (m, 2H, H-3', H-4'), 3.84 (s, 3H, OCH $), 3.86$ (m, 1H, H-5a), 4.35 (dd, 1H, $J=$ $11.4, J=8.3 \mathrm{~Hz}, \mathrm{H}-5 \mathrm{~b}), 4.47$ (m, 1H, H-4), 4.53 (d, 1H, $\left.J=11.3 \mathrm{~Hz}, \mathrm{CH}_{2} \mathrm{MPM}\right), 4.62$ (d, 2H, $J=$ $\left.12.2 \mathrm{~Hz}, \mathrm{CH}_{2} \mathrm{Ph}\right), 4.65\left(\mathrm{~d}, 1 \mathrm{H}, J=12.8 \mathrm{~Hz}, \mathrm{CH}_{2} \mathrm{Ph}\right), 4.69$ (d, $\left.1 \mathrm{H}, J=12.2 \mathrm{~Hz}, \mathrm{CH}_{2} \mathrm{Ph}\right), 4.72(\mathrm{~d}, 1 \mathrm{H}$, $\left.J=11.0 \mathrm{~Hz}, \mathrm{CH}_{2} \mathrm{Ph}\right), 4.80\left(\mathrm{~d}, 1 \mathrm{H}, J=11.4 \mathrm{~Hz}, \mathrm{CH}_{2} \mathrm{MPM}\right), 4.81$ (d, 1H, J= $7.7 \mathrm{~Hz}, \mathrm{H}-1$ '), 4.85 (m, 2H, H-1, $\mathrm{CH}_{2} \mathrm{Ph}$ ), 5.32 (t, $1 \mathrm{H}, J=8.2 \mathrm{~Hz}, \mathrm{H}-2$ ') 5.45 (dd, 1H, J= 5.3, J=3.0 Hz, H-2), 5.47 (dd, $1 \mathrm{H}, J=5.4, J=2.9 \mathrm{~Hz}, \mathrm{H}-3), 6.82(\mathrm{~d}, 2 \mathrm{H}, J=8.6 \mathrm{~Hz}, \mathrm{Ar}-\mathrm{H}), 7.10-7.60$ (m, 26H, Ar-H), 7.67 (d, 2H, $J=7.2 \mathrm{~Hz}, \mathrm{Ar}-\mathrm{H}), 7.87(\mathrm{~d}, 2 \mathrm{H}, J=7.3 \mathrm{~Hz}, \mathrm{Ar}-\mathrm{H}), 8.30(\mathrm{~d}, 2 \mathrm{H}, J=7.2 \mathrm{~Hz}, \mathrm{Ar}-\mathrm{H}) .{ }^{13} \mathrm{C} \mathrm{NMR}\left(\mathrm{CDCl}_{3}\right)$ : $\delta 55.2\left(\mathrm{OCH}_{3}\right), 60.9(\mathrm{C}-5), 68.6\left(\mathrm{C}-6\right.$ ') 69.0 (C-2), $69.3\left(\mathrm{CH}_{2} \mathrm{MPM}\right), 69.7(\mathrm{C}-3), 70.7(\mathrm{C}-4), 73.4$ $\left(\mathrm{CH}_{2} \mathrm{Ph}, \mathrm{C}-2\right.$ ') $74.9\left(\mathrm{CH}_{2} \mathrm{Ph}\right), 75.0\left(\mathrm{CH}_{2} \mathrm{Ph}\right), 75.2\left(\mathrm{C}^{\prime} 5\right.$ '), 77.8 (C-4'), 82.7 (C-3'), $97.0(\mathrm{C}-1), 100.0$ $\left(\mathrm{C}^{\prime} 1^{\prime}\right), 113.7(\mathrm{CH}), 127.6(\mathrm{CH}), 127.7(\mathrm{CH}), 127.7(\mathrm{CH}), 127.8(\mathrm{CH}), 128.0(\mathrm{CH}), 128.1(\mathrm{CH})$, $128.2(\mathrm{CH}), 128.3(\mathrm{CH}), 128.4(\mathrm{CH}), 128.4(\mathrm{CH}), 129.1(\mathrm{C}), 129.3(\mathrm{C}), 129.4(\mathrm{C}), 129.6(\mathrm{CH})$, $129.7(\mathrm{CH}), 129.8(\mathrm{CH}), 129.8(\mathrm{CH}), 159.2(\mathrm{C}), 164.7(\mathrm{CO}), 164.9(\mathrm{CO}), 165.4(\mathrm{CO})$. Anal. Calcd for $\mathrm{C}_{61} \mathrm{H}_{58} \mathrm{O}_{14}$ : C, 72.18; H, 5.76. Found: C, 72.07; H, 5.80.

\subsection{2. $2,3,4-T r i-O$-benzoyl- $\beta$-D-xylopyranosyl- $(1 \rightarrow 2)-3,4-d i-O$-benzoyl- $\beta$-L-arabinopyranosyl}

trichloroacetimidate (27). General method. In a typical experiment, trifluoroacetic acid $(4.0 \mathrm{~mL}$, $52.5 \mathrm{mmol}, 20 \mathrm{eq})$ and $\mathrm{H}_{2} \mathrm{O}(0.56 \mathrm{~mL}, 31.6 \mathrm{mmol}, 12 \mathrm{eq})$ were added to a solution of the disaccharide $13(2.42 \mathrm{~g}, 2.6 \mathrm{mmol})$ in $\mathrm{CH}_{2} \mathrm{Cl}_{2}(90 \mathrm{~mL})$. The reaction was vigorously stirred overnight before being washed with $\mathrm{H}_{2} \mathrm{O}, \mathrm{NaHCO}_{3}$ (sat.), and $\mathrm{NaCl}$ (sat.). The dried solution $\left(\mathrm{Na}_{2} \mathrm{SO}_{4}\right)$ was then evaporated under reduced pressure and the residue taken up in $\mathrm{CH}_{2} \mathrm{Cl}_{2}(30 \mathrm{~mL})$. Trichloroacetonitrile $(1.3 \mathrm{~mL}, 12.8 \mathrm{mmol}, 5 \mathrm{eq})$ was added, followed by DBU (0.04 mL, 0.26 mmol, $0.1 \mathrm{eq}$ ), and the reaction was stirred overnight. The reaction was then evaporated and the crude residue was purified by column chromatography (cyclohexane/EtOAc/Et $3 \mathrm{~N}$ 9:1:0.1) to give $2.20 \mathrm{~g}(89 \%)$ of 27 as a white amorphous solid. $\mathrm{R}_{f}=0.53$ (cyclohexane/EtOAc $\left.6: 4\right) .[\alpha]_{\mathrm{D}}=+94.6^{\circ}$ $\left(c 1, \mathrm{CHCl}_{3}\right) .{ }^{1} \mathrm{H} \mathrm{NMR}\left(\mathrm{CDCl}_{3}\right): \delta 3.81(\mathrm{dd}, 1 \mathrm{H}, J=12.3, J=6.7 \mathrm{~Hz}, \mathrm{H}-5$ ') $4.09(\mathrm{dd}, 1 \mathrm{H}, J=13.2$, $J=1.6 \mathrm{~Hz}, \mathrm{H}-5$ ), 4.37 (brd, $1 \mathrm{H}, J=12.9 \mathrm{~Hz}, \mathrm{H}-5$ ), 4.54 (dd, $1 \mathrm{H}, J=12.4, J=4.4 \mathrm{~Hz}, \mathrm{H}-5$ '), 4.62 (dd, 1H, $J=10.4, J=3.6 \mathrm{~Hz}, \mathrm{H}-2), 5.17$ (d, 1H, J= 5.4 Hz, H-1'), 5.34 (m, 1H, H-4'), 5.37 (dd, 1H, 
$\left.J=7.8, J=5.5 \mathrm{~Hz}, \mathrm{H}-2^{\prime}\right), 5.71(\mathrm{t}, 1 \mathrm{H}, J=7.5 \mathrm{~Hz}, \mathrm{H}-3$ '), 5.77 (dd, 1H, $J=10.4, J=3.5 \mathrm{~Hz}, \mathrm{H}-3$ ), $5.82(\mathrm{~m}, 1 \mathrm{H}, \mathrm{H}-4), 6.79$ (d, 1H, $J=3.5 \mathrm{~Hz}, \mathrm{H}-1), 7.12$ (t, 2H, $J=7.7 \mathrm{~Hz}, \operatorname{Ar}-\mathrm{H}), 7.30-7.67$ (m, 15H, Ar-H), 7.80 (d, 2H, $J=8.3 \mathrm{~Hz}, \mathrm{Ar}-\mathrm{H}), 7.91$ (d, 2H, $J=8.4 \mathrm{~Hz}, \mathrm{Ar}-\mathrm{H}), 8.04$ (d, 2H, J = 8.4 Hz, Ar$\mathrm{H}), 8.08(\mathrm{~d}, 2 \mathrm{H}, J=8.4 \mathrm{~Hz}, \mathrm{Ar}-\mathrm{H}), 8.82(\mathrm{~s}, 1 \mathrm{H}, \mathrm{NH}) .{ }^{13} \mathrm{C} \mathrm{NMR}\left(\mathrm{CDCl}_{3}\right): \delta 61.7(\mathrm{C}-5$ '), $62.8(\mathrm{C}-5)$, 69.3 (C-3), 69.4 (C-4'), 69.5 (C-4), 70.2 (C-2'), 70.2 (C-3’), 74.0 (C-2), 95.9 (C-1), 101.7 (C-1'), $128.1(\mathrm{CH}), 128.3(\mathrm{CH}), 128.3(\mathrm{CH}), 128.4(\mathrm{CH}), 128.5(\mathrm{CH}), 128.8(\mathrm{C}), 120.0(\mathrm{C}), 129.1(\mathrm{C})$, $129.5(\mathrm{CH}), 129.5(\mathrm{CH}), 129.5(\mathrm{C}), 129.8(\mathrm{CH}), 129.9(\mathrm{CH}), 132.9(\mathrm{C}), 133.1(\mathrm{C}), 133.3(\mathrm{C}), 133.4$ (C), $161.1(C=\mathrm{NH}), 164.7(\mathrm{CO}), 165.1(\mathrm{CO}), 165.4(\mathrm{CO}), 165.5$ (CO). Anal. Calcd for $\mathrm{C}_{47} \mathrm{H}_{38} \mathrm{Cl}_{3} \mathrm{NO}_{14}$ : C, 59.60; H, 4.04; N, 1.48. Found: C, 59.45; H, 4.07; N, 1.40 .

\subsection{3. $2,3,4-T r i-O$-benzoyl- $\beta$-D-xylopyranosyl- $(1 \rightarrow 3)$-2,4-di- $O$-benzoyl- $\beta$-L-arabinopyranosyl}

trichloroacetimidate (28). This compound was prepared using the general method described for 27. Deprotection of disaccharide $14(0.65 \mathrm{~g}, 0.7 \mathrm{mmol})$ followed by trichloroacetimidate formation gave $0.53 \mathrm{~g}(80 \%)$ of $28 . \mathrm{R}_{f}=0.55$ (cyclohexane/EtOAc $\left.6: 4\right) .[\alpha]_{\mathrm{D}}=+45.6^{\circ}\left(c 0.5, \mathrm{CHCl}_{3}\right) .{ }^{1} \mathrm{H}$ $\operatorname{NMR}\left(\mathrm{CDCl}_{3}\right): \delta 3.81(\mathrm{dd}, 1 \mathrm{H}, J=12.5, J=5.7 \mathrm{~Hz}, \mathrm{H}-5$ '), $4.20(\mathrm{dd}, 1 \mathrm{H}, J=13.4, J=2.0 \mathrm{~Hz}, \mathrm{H}-5)$, 4.31 (brd, $1 \mathrm{H}, J=12.9 \mathrm{~Hz}, \mathrm{H}-5), 4.47$ (dd, $1 \mathrm{H}, J=12.6, J=4.0 \mathrm{~Hz}, \mathrm{H}-5$ '), 4.68 (dd, 1H, $J=10.3, J$ $=3.4 \mathrm{~Hz}, \mathrm{H}-3), 5.26$ (m, 2H, H-1', H-4'), 5.34 (dd, 1H, $\left.J=7.0, J=4.8 \mathrm{~Hz}, \mathrm{H}-2^{\prime}\right), 5.67$ (t, 1H, $J=$ $6.8 \mathrm{~Hz}, \mathrm{H}-3$ ') 5.78 (m, 1H, H-4), 5.85 (dd, 1H, $J=10.3, J=3.6 \mathrm{~Hz}, \mathrm{H}-2), 6.74$ (d, 1H, J=3.6 Hz, $\mathrm{H}-1), 7.17$ (t, 2H, $J=7.6 \mathrm{~Hz}, \mathrm{Ar}-\mathrm{H}), 7.27$ (t, 2H, $J=7.6 \mathrm{~Hz}, \mathrm{Ar}-\mathrm{H}), 7.35$ (t, 2H, $J=7.7 \mathrm{~Hz}, \mathrm{Ar}-\mathrm{H})$, 7.40-7.69 (m, 11H, Ar-H), 7.87 (d, 2H, J=8.3 Hz, Ar-H), 7.92 (d, 2H, J = 8.4 Hz, Ar-H), 8.04 (d, $2 \mathrm{H}, J=8.4 \mathrm{~Hz}, \mathrm{Ar}-\mathrm{H}), 8.17(\mathrm{~d}, 2 \mathrm{H}, J=8.4 \mathrm{~Hz}, \mathrm{Ar}-\mathrm{H}), 8,60(\mathrm{~s}, 1 \mathrm{H}, \mathrm{N} H) .{ }^{13} \mathrm{C} \mathrm{NMR}\left(\mathrm{CDCl}_{3}\right): \delta 61.2$ (C-5'), 63.1 (C-5), 69.0 (C-4’), 69.5 (C-2), 69.7 (C-3'), 69.9 (C-2'), 71.4 (C-4), 73.5 (C-3), 94.3 (C1-), $\left.101.4(\mathrm{C}-1 ')^{\prime}\right), 128.1(\mathrm{CH}), 128.3(\mathrm{CH}), 128.3(\mathrm{CH}), 128.4(\mathrm{CH}), 128.5(\mathrm{CH}), 128.8(\mathrm{C}), 128.8$ (C), $129.2(\mathrm{C}), 129.5(\mathrm{CH}), 129.5(\mathrm{C}), 129.6(\mathrm{CH}), 129.6(\mathrm{CH}), 129.9(\mathrm{CH}), 130.0(\mathrm{CH}), 132.9$ $(\mathrm{CH}), 133.2(\mathrm{CH}), 133.3(\mathrm{CH}), 133.3(\mathrm{CH}), 133.4(\mathrm{CH}), 160.5(\mathrm{C}=\mathrm{NH}), 164.6(\mathrm{CO}), 165.2(\mathrm{CO})$, 165.3 (CO), 165.4 (CO), 166.1 (CO). Anal. Calcd for $\mathrm{C}_{47} \mathrm{H}_{38} \mathrm{Cl}_{3} \mathrm{NO}_{14}$ : C, 59.60; H, 4.04; N, 1.48 . Found: C, 59.25; H, 3.93; N, 1.35 .

\subsection{4. $2,3,4-$ Tri- $O$-benzoyl- $\beta$-D-xylopyranosyl-( $1 \rightarrow 4)$-2,3-di- $O$-benzoyl- $\beta$-L-arabinopyranosyl} trichloroacetimidate (29). This compound was prepared using the general method described for 27. Deprotection of disaccharide $15(0.65 \mathrm{~g}, 0.7 \mathrm{mmol})$ followed by trichloroacetimidate formation gave $0.56 \mathrm{~g}(83 \%)$ of 29. $\mathrm{R}_{f}=0.55$ (cyclohexane/EtOAc 6:4). $[\alpha]_{\mathrm{D}}=+50.1^{\circ}\left(c 1, \mathrm{CHCl}_{3}\right) .{ }^{1} \mathrm{H} \mathrm{NMR}$ $\left(\mathrm{CDCl}_{3}\right): \delta 3.86(\mathrm{dd}, 1 \mathrm{H}, J=12.5, J=5.1 \mathrm{~Hz}, \mathrm{H}-5$ ') $, 4.28(\mathrm{dd}, 1 \mathrm{H}, J=12.7, J=1.5 \mathrm{~Hz}, \mathrm{H}-5), 4.37$ (brd, 1H, $J=12.7$ Hz, H-5), 4.59 (m, 2H, H-4, H-5'), 5.13 (d, 1H, J= 3.8 Hz, H-1'), 5.34 (m, 1H, H-4'), 5.54 (dd, 1H, J=5.5, $J=4.1 \mathrm{~Hz}, \mathrm{H}-2^{\prime}$ ') 5.77 (t, 1H, $J=5.7 \mathrm{~Hz}, \mathrm{H}-3$ '), 5.87 (dd, 1H, $J=10.7$, 
$J=3.4 \mathrm{~Hz}, \mathrm{H}-2), 5.93(\mathrm{dd}, 1 \mathrm{H}, J=10.7, J=2.9 \mathrm{~Hz}, \mathrm{H}-3), 6.86$ (d, 1H, $J=3.3 \mathrm{~Hz}, \mathrm{H}-1), 7.24-7.62$ (m, 15H, Ar-H), 7.90 (d, 2H, J=7.4 Hz, Ar-H), 7.94 (d, 2H, J=7.4 Hz, Ar-H), 7.96 (d, 2H, J = 7.4 $\mathrm{Hz}, \mathrm{Ar}-\mathrm{H}), 8.04$ (d, 2H, J=7.4 Hz, Ar-H), 8.18 (d, 2H, J=7.4 Hz, Ar-H), 8.66 (s, 1H, NH). ${ }^{13} \mathrm{C}$ NMR ( $\left.\mathrm{CDCl}_{3}\right): \delta 60.4$ (C-5’), 64.6 (C-5), 68.1 (C-2), 68.2 (C-4'), 68.5 (C-3’), 69.4 (C-2', C-3), 74.8 (C-4), 94.2 (C-1), 100.5 (C-1'), $128.2(\mathrm{CH}), 128.3(\mathrm{CH}), 128.3(\mathrm{CH}), 128.4(\mathrm{CH}), 128.5(\mathrm{CH})$, 128.7 (C), 128.9 (C), 129.0 (C), 129.1 (C), 129.2 (C), $129.7(\mathrm{CH}), 129.7(\mathrm{CH}), 129.8(\mathrm{CH}), 130.0$ $(\mathrm{CH}), 133.1(\mathrm{CH}), 133.2(\mathrm{CH}), 133.3(\mathrm{CH}), 133.5(\mathrm{CH}), 160.8(\mathrm{C}=\mathrm{NH}), 164.9(\mathrm{CO}), 165.1(\mathrm{CO})$, 165.2 (CO), 165.4 (CO), 165.9 (CO). Anal. Calcd for $\mathrm{C}_{47} \mathrm{H}_{38} \mathrm{Cl}_{3} \mathrm{NO}_{14}$ : C, 59.60; H, 4.04; N, 1.48. Found: C, 59.23; H, 4.04; N, 1.58 .

\subsection{2- $O$-Benzoyl-3,4,6-tri- $O$-benzyl- $\beta$-D-glucopyranosyl-(1 $\rightarrow 2)-3,4$-di- $O$-benzoyl- $\beta$-L-} arabinopyranosyl trichloroacetimidate (30). This compound was prepared using the general method described for 27. Deprotection of disaccharide 23 (6.47g, $6.4 \mathrm{mmol})$ followed by trichloroacetimidate formation gave $4.6 \mathrm{~g}(78 \%)$ of $30 . \mathrm{R}_{f}=0.64$ (cyclohexane/ EtOAc 6:4). $[\alpha]_{\mathrm{D}}=$

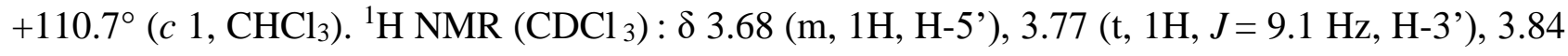
(t, 1H, $J=9.4 \mathrm{~Hz}, \mathrm{H}-4^{\prime}$ ), 3.87 (m, 2H, H-6'a/b), 4.07 (dd, 1H, J= 13.3, J=1.8 Hz, H-5a), 4.31 (d, $1 \mathrm{H}, J=12.9 \mathrm{~Hz}, \mathrm{H}-5 \mathrm{~b}), 4.56$ (m, 1H, H-2), 4.57 (d, $\left.1 \mathrm{H}, J=11.3 \mathrm{~Hz}, \mathrm{CH}_{2} \mathrm{Ph}\right), 4.66$ (m, 3H, CH $\mathrm{H}_{2} \mathrm{Ph}$ ), $4.71\left(\mathrm{~d}, 1 \mathrm{H}, J=11.9 \mathrm{~Hz}, \mathrm{CH}_{2} \mathrm{Ph}\right), 4.84$ (d, $\left.1 \mathrm{H}, J=10.8 \mathrm{~Hz}, \mathrm{CH}_{2} \mathrm{Ph}\right), 4.87$ (d, 1H, J= 7.8 Hz, H-1'), $5.25(\mathrm{dd}, 1 \mathrm{H}, J=9.2, J=7.9 \mathrm{~Hz}, \mathrm{H}-2$ ') 5.68 (dd, 1H, $J=10.4, J=3.5 \mathrm{~Hz}, \mathrm{H}-3), 3.78$ (m, 1H, H-4), 6.79 (d, 1H, $J=3.6 \mathrm{~Hz}, \mathrm{H}-1), 7.07-7.65$ (m, 25H, Ar-H), $7.74(\mathrm{dd}, 2 \mathrm{H}, J=8.3, J=1.2 \mathrm{~Hz}, \mathrm{Ar}-\mathrm{H})$, $8.05(\mathrm{dd}, 2 \mathrm{H}, J=8.3, J=1.3 \mathrm{~Hz}, \mathrm{Ar}-\mathrm{H}), 8.11(\mathrm{dd}, 1 \mathrm{H}, J=8.3, J=1.3 \mathrm{~Hz}, \mathrm{Ar}-\mathrm{H}), 8.64(\mathrm{~s}, 1 \mathrm{H}, \mathrm{NH})$. ${ }^{13} \mathrm{C} \mathrm{NMR}\left(\mathrm{CDCl}_{3}\right): \delta 62.7(\mathrm{C}-5), 68.9(\mathrm{C}-6$ ') $, 69.4(\mathrm{C}-4), 69.7(\mathrm{C}-3), 73.0(\mathrm{C}-2), 73.4(\mathrm{C}-2$ ') 73.7 $\left(\mathrm{CH}_{2} \mathrm{Ph}\right), 75.0\left(\mathrm{CH}_{2} \mathrm{Ph}\right), 75.1\left(\mathrm{CH}_{2} \mathrm{Ph}\right), 75.4(\mathrm{C}-5$ ') $77.7(\mathrm{C}-4$ '), $82.5(\mathrm{C}-3$ '), $96.2(\mathrm{C}-1), 101.6(\mathrm{C}-$ 1'), $127.6(\mathrm{CH}), 127.7(\mathrm{CH}), 127.7(\mathrm{CH}), 127.9(\mathrm{CH}), 128.0(\mathrm{CH}), 128.2(\mathrm{CH}), 128.2(\mathrm{CH}), 128.4$ $(\mathrm{CH}), 128.5(\mathrm{CH}), 129.1(\mathrm{C}), 129.2(\mathrm{CH}), 129.3(\mathrm{C}), 129.5(\mathrm{CH}), 129.6(\mathrm{CH}), 129.6(\mathrm{C}), 129.8$ $(\mathrm{CH}), 132.6(\mathrm{CH}), 132.9(\mathrm{CH}), 133.0(\mathrm{CH}), 133.4(\mathrm{CH}), 137.6(\mathrm{C}), 137.9(\mathrm{C}), 138.1(\mathrm{C}), 161.2$ $(C=\mathrm{NH}), 164.6(\mathrm{CO}), 164.9(\mathrm{CO}), 165.5(\mathrm{CO})$. Anal. Calcd for $\mathrm{C}_{55} \mathrm{H}_{50} \mathrm{Cl}_{3} \mathrm{NO}_{13}: \mathrm{C}, 63.56$; H, 4.85; N, 1.35. Found: C, 63.80; H, 4.59; N, 1.30 .

4.16. 2- $O$-Benzoyl-3,4,6-tri- $O$-benzyl- $\beta$-D-glucopyranosyl-( $1 \rightarrow 3)$-2,4-di- $O$-benzoyl- $\beta$-Larabinopyranosyl trichloroacetimidate (31). This compound was prepared using the general method described for 27. Deprotection of disaccharide 24 (0.97 g, $1.0 \mathrm{mmol})$ followed by trichloroacetimidate formation gave $0.66 \mathrm{~g}(74 \%)$ of 31. $\mathrm{R}_{f}=0.65$ (cyclohexane/ EtOAc 6:4). $[\alpha]_{\mathrm{D}}=$ $\left.+68.5^{\circ}\left(c_{1} 1, \mathrm{CHCl}_{3}\right) .{ }^{1} \mathrm{H} \mathrm{NMR}_{\left(\mathrm{CDCl}_{3}\right)}\right): \delta 3.66$ (m, 1H, H-5'), 3.77 (m, 4H, H-3', H-4', H-6'a, H6’b), 4.20 (m, 2H, H-5a, H-5b), 4.51 (d, $1 \mathrm{H}, J=11.9, \mathrm{CH}_{2} \mathrm{Ph}$ ), 4.56 (d, 3H, $J=11.5, \mathrm{CH}_{2} \mathrm{Ph}$ ), 4.60 
(dd, $1 \mathrm{H}, J=10.2, J=3.5, \mathrm{H}-3), 4.65$ (d, 1H, $\left.J=11.1, \mathrm{CH}_{2} \mathrm{Ph}\right), 4.77$ (d, $\left.1 \mathrm{H}, J=10.8, \mathrm{CH}_{2} \mathrm{Ph}\right), 4.93$ (d, 1H, J= 7.6, H-1'), 5.24 (m, 1H, H-2'), 5.73 (m, 1H, H-4), 5.75 (dd, 1H, J=10.2, J= 3.5, H-2), 6.65 (d, 1H, $J=3.6, \mathrm{H}-1), 7.06-7.62$ (m, 24H, Ar-H), 7.66 (dd, 2H, $J=8.3, J=1.3$, Ar-H), 7.84 (dd, $2 \mathrm{H}, J=8.4, J=1.2, \mathrm{Ar}-\mathrm{H}), 8.15(\mathrm{dd}, 2 \mathrm{H}, J=8.4, J=1.4, \mathrm{Ar}-\mathrm{H}), 8.52(\mathrm{~s}, 1 \mathrm{H}, \mathrm{N} H) .{ }^{13} \mathrm{C} \mathrm{NMR}$ $\left(\mathrm{CDCl}_{3}\right): \delta 62.9(\mathrm{C}-5), 68.9\left(\mathrm{C}-6\right.$ ') 69.6 (C-2), $71.4(\mathrm{C}-4), 73.0(\mathrm{C}-3), 73.4\left(\mathrm{C}-2\right.$ '), $73.5\left(\mathrm{CH}_{2} \mathrm{Ph}\right)$, $74.7\left(\mathrm{CH}_{2} \mathrm{Ph}\right), 75.0\left(\mathrm{CH}_{2} \mathrm{Ph}\right), 75.3$ (C-5'), 77.6 (C-4'), 82.5 (C-3'), 94.2 (C-1), 101.5 (C-1'), 127.5 $(\mathrm{CH}), 127.5(\mathrm{CH}), 127.7(\mathrm{CH}), 127.8(\mathrm{CH}), 128.0(\mathrm{CH}), 128.1(\mathrm{CH}), 128.1(\mathrm{CH}), 128.2(\mathrm{CH})$, $128.3(\mathrm{CH}), 128.4(\mathrm{CH}), 128.4(\mathrm{CH}), 128.9(\mathrm{C}), 129.3(\mathrm{CH}), 129.4(\mathrm{C}), 129.6(\mathrm{CH}), 129.9(\mathrm{C})$, $130.0(\mathrm{CH}), 132.7(\mathrm{CH}), 132.9(\mathrm{CH}), 133.1(\mathrm{CH}), 137.6(\mathrm{C}), 137.8(\mathrm{C}), 138.1(\mathrm{C}), 160.5(C=\mathrm{NH})$, 164.6 (CO), 165.1 (CO), 166.3 (CO). Anal. Calcd for $\mathrm{C}_{55} \mathrm{H}_{50} \mathrm{Cl}_{3} \mathrm{NO}_{13}$ : C, 63.56; H, 4.85; N, 1.35 . Found: C, 63.17; H, 4.56; N, 1.38.

4.17. 2- $O$-benzoyl-3,4,6-tri- $O$-benzyl- $\beta$-D-glucopyranosyl-( $1 \rightarrow 4)-2,3$-di- $O$-benzoyl- $\beta$-Larabinopyranosyl trichloroacetimidate (32). This compound was prepared using the general method described for 27. Deprotection of disaccharide 25 (0.65 g, $0.6 \mathrm{mmol})$ followed by trichloroacetimidate formation gave $0.52 \mathrm{~g}(77 \%)$ of 32. $\mathrm{R}_{f}=0.63$ (cyclohexane/ EtOAc 6:4). $[\alpha]_{\mathrm{D}}=$ $+66.3^{\circ}\left(c^{1}, \mathrm{CHCl}_{3}\right) .{ }^{1} \mathrm{H}$ NMR $\left(\mathrm{CDCl}_{3}\right): \delta 3.54\left(\mathrm{~m}, 1 \mathrm{H}, \mathrm{H}-5^{\prime}\right), 3.72(\mathrm{~m}, 2 \mathrm{H}, \mathrm{H}-6$ 'a/b), $3.81(\mathrm{~m}, 2 \mathrm{H}$, H-3', H-4'), 4.24 (d, 1H, J=12.5 Hz, H-5a), 4.32 (dd, 1H, J= 12.6, J=1.7 Hz, H-5b), 4.54 (m, 1H, $\mathrm{H}-4), 4.62\left(\mathrm{~m}, 3 \mathrm{H}, \mathrm{CH}_{2} \mathrm{Ph}\right), 4.70\left(\mathrm{~d}, 1 \mathrm{H}, J=11.0 \mathrm{~Hz}, \mathrm{CH}_{2} \mathrm{Ph}\right), 4.77$ (d, $1 \mathrm{H}, J=11.9 \mathrm{~Hz}, \mathrm{CH} \mathrm{Ph} \mathrm{Ph}$, 4.79 (d, 1H, J=7.9 Hz, H-1'), 4.85 (d, 1H, J=10.9 Hz, CH $\left.H_{2} \mathrm{Ph}\right), 5.47$ (m, 1H, H-2'), 5.78 (m, 2H, H-2, H-3), 6.79 (d, 1H, J=1.7 Hz, H-1), 7.17-7.54 (m, 24H, Ar-H), 7.78 (d, 2H, J= 7.3 Hz, Ar-H), $7.91(\mathrm{~d}, 2 \mathrm{H}, J=7.3 \mathrm{~Hz}, \mathrm{Ar}-\mathrm{H}), 7.97$ (d, 2H, $J=7.3 \mathrm{~Hz}, \mathrm{Ar}-\mathrm{H}), 8.60(\mathrm{~s}, 1 \mathrm{H}, \mathrm{N} H) .{ }^{13} \mathrm{C} \mathrm{NMR}\left(\mathrm{CDCl}_{3}\right)$ : $\delta 64.8$ (C-5), 67.8 (C-2), 68.7 (C-6’), 69.9 (C-3), $73.5\left(\mathrm{CH}_{2} \mathrm{Ph}\right), 73.7(\mathrm{C}-2$ ') $, 74.8(\mathrm{C}-4), 75.0(2 \times$ $\left.\mathrm{CH}_{2} \mathrm{Ph}\right), 75.1$ (C-5'), 77.8 (C-4'), 82.8 (C-3'), 94.6 (C-1), $101.9\left(\mathrm{C}-1^{\prime}\right), 127.6(\mathrm{CH}), 127.7(\mathrm{CH})$, $127.9(\mathrm{CH}), 128.3(\mathrm{CH}), 128.5(\mathrm{CH}), 128.8(\mathrm{C}), 129.1(\mathrm{C}), 129.5(\mathrm{CH}), 129.7(\mathrm{CH}), 129.7(\mathrm{CH})$, $129.8(\mathrm{C}), 132.7(\mathrm{CH}), 133.1(\mathrm{CH}), 133.2(\mathrm{CH}), 137.7(\mathrm{C}), 137.7(\mathrm{C}), 137.8(\mathrm{C}), 138.0(\mathrm{C}), 160.6$ $(C=\mathrm{NH}), 164.9(\mathrm{CO}), 165.0(\mathrm{CO}), 166.0(\mathrm{CO})$. Anal. Calcd for $\mathrm{C}_{55} \mathrm{H}_{50} \mathrm{Cl}_{3} \mathrm{NO}_{13}: \mathrm{C}, 63.56$; H, 4.85; N, 1.35. Found: C, 63.88; H, 5.14; N, 1.26.

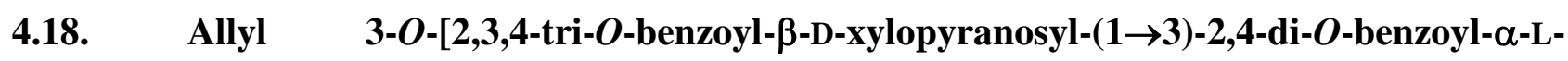
arabinopyranosyl]-23- $O$-benzoylhederagenate (34). General coupling method. In a typical experiment, allyl hederagenate $3^{5}(0.150 \mathrm{~g}, 0.24 \mathrm{mmol})$, trichloroacetimidate $28(0.35 \mathrm{~g}, 0.37$ mmol, 1.5 eq) and $4 \AA$ powdered molecular sieves $(1 \mathrm{~g})$ were stirred for $1 \mathrm{~h}$ at room temperature in $\mathrm{CH}_{2} \mathrm{Cl}_{2}$ (4 mL). The mixture was cooled to $-20{ }^{\circ} \mathrm{C}$ for 30 minutes followed by the dropwise addition of a $0.1 \mathrm{M}$ solution of TMSOTf in $\mathrm{CH}_{2} \mathrm{Cl}_{2}(0.12 \mathrm{~mL}, 0.012 \mathrm{mmol}, 0.05 \mathrm{eq})$. After $6 \mathrm{~h}$ at -20 
${ }^{\circ} \mathrm{C}$ the reaction was quenched with triethylamine, filtered through Celite and evaporated. Purification by column chromatography (toluene/acetone 99:1 to 98.5:1.5) gave $0.32 \mathrm{~g}$ (95\%) of saponin 34 as a white foam. $\mathrm{R}_{f}=0.63$ (toluene/acetone 9:1). $[\alpha]_{\mathrm{D}}=+53.1^{\circ}\left(c 1, \mathrm{CHCl}_{3}\right) .{ }^{1} \mathrm{H} \mathrm{NMR}$ $\left(\mathrm{CDCl}_{3}\right): \delta 0.63(\mathrm{~s}, 3 \mathrm{H}, \mathrm{H}-24), 0.73$ (s, 3H, H-26), 0.90-1.98 (m, 22H, H-1, H-2, H-5, H-6, H-7, H9, H-11, H-15, H-16, H-19, H-21, H-22), 0.93 (s, 3H, H-25), 0.94 (s, 3H, H-29), 0.96 (s, 3H, H-30), 1.05 (s, 3H, H-27), 2.91 (dd, 1H, $J=13.7, J=3.8 \mathrm{~Hz}, \mathrm{H}-18), 3.56$ (dd, $1 \mathrm{H}, J=11.6, J=4.7 \mathrm{~Hz}, \mathrm{H}-$ 3), 3.66 (dd, 1H, $J=12.5, J=5.7$ Hz, H-5”), 3.69 (brd, 1H, $J=13.6 \mathrm{~Hz}, \mathrm{H}-5$ '), 3.83 (d, 1H, J= 11.5 Hz, H-23), 4.09 (d, 1H, $J=11.6$ Hz, H-23), 4.29 (m, 2H, H-3', H-5'), 4.39 (dd, 1H, J= 12.5, $J=$ $3.8 \mathrm{~Hz}, \mathrm{H}-5$ ”), 4.55 (m, 2H, $\mathrm{CH}_{2} \mathrm{CH}=\mathrm{CH}_{2}$ ), 4.65 (d, 1H, J= $7.1 \mathrm{~Hz}, \mathrm{H}-1$ '), 5.14 (d, 1H, J= $4.5 \mathrm{~Hz}$, H-1"), 5.19 (m, 1H, H-4"), 5.23 (dd, 1H, $J=10.8, J=0.8 \mathrm{~Hz}, \mathrm{CH}_{2} \mathrm{CH}=\mathrm{CH}_{2}$ ), 5.27 (dd, 1H, $J=6.7$, $J=4.7 \mathrm{~Hz}, \mathrm{H}-2$ ') 5.34 (m, 2H, $\left.\mathrm{CH}_{2} \mathrm{CH}=\mathrm{CH}_{2}, \mathrm{H}-12\right), 5.55$ (m, 1H, H-4'), 5.60 (t, 1H, J=6.5 Hz, H3”), 5.71 (dd, $1 \mathrm{H}, J=8.9, J=7.4 \mathrm{~Hz}, \mathrm{H}-2$ '), $5.92\left(\mathrm{~m}, 1 \mathrm{H}, \mathrm{CH}_{2} \mathrm{CH}=\mathrm{CH}_{2}\right), 7.23$ (t, 2H, $J=7.7 \mathrm{~Hz}$, Ar-H), 7.30-7.65 (m, 16H, Ar-H), 7.70 (d, 2H, J = 7.6 Hz, Ar-H), 7.94 (d, 2H, J = 7.8 Hz, Ar-H), $7.96(\mathrm{~d}, 2 \mathrm{H}, J=7.6 \mathrm{~Hz}, \mathrm{Ar}-\mathrm{H}), 8.01$ (d, 2H, $J=7.4 \mathrm{~Hz}, \mathrm{Ar}-\mathrm{H}), 8.04$ (d, 2H, $J=7.3 \mathrm{~Hz}, \mathrm{Ar}-\mathrm{H}), 8.16$ $(\mathrm{d}, 2 \mathrm{H}, J=7.4 \mathrm{~Hz}, \mathrm{Ar}-\mathrm{H}) .{ }^{13} \mathrm{C} \mathrm{NMR}\left(\mathrm{CDCl}_{3}\right): \delta 12.5$ (C-24), 15.5 (C-25), 16.9 (C-26), $17.9(\mathrm{C}-6)$, 22.9 (C-16), 23.4 (C-11), 23.6 (C-30), 25.3 (C-27, C-2), 27.5 (C-15), 30.6 (C-20), 32.3 (C-7, C-22), 33.1 (C-29), 33.8 (C-21), 36.4 (C-10), 38.3 (C-1), 39.3 (C-8), 41.3 (C-18), 41.6 (C-14), 42.1 (C-4), 45.8 (C-19), 46.7 (C-17), 48.0 (C-9), 48.1 (C-5), 60.8 (C-5”), 63.2 (C-5'), 64.8 ( $\left.\mathrm{CH}_{2} \mathrm{CH}=\mathrm{CH}_{2}\right), 65.3$ (C-23), 68.8 (C-4”), 69.5 (C-3”), 69.8 (C-2”), 71.0 (C-4’), 71.3 (C-2'), 77.1 (C-3’), 83.4 (C-3), 100.7 (C-1"), 103.2 (C-1'), $117.7\left(\mathrm{CH}_{2} \mathrm{CH}=\mathrm{CH}_{2}\right), 122.4(\mathrm{C}-12), 128.0(\mathrm{CH}), 128.3(\mathrm{CH}), 128.3$ $(\mathrm{CH}), 128.4(\mathrm{CH}), 128.6(\mathrm{CH}), 128.9(\mathrm{C}), 129.0(\mathrm{C}), 129.2(\mathrm{C}), 129.2(\mathrm{C}), 129.4(\mathrm{CH}), 129.7(\mathrm{CH})$, $129.7(\mathrm{CH}), 129.8(\mathrm{CH}), 129.8(\mathrm{CH}), 130.0(\mathrm{CH}), 130.4(\mathrm{C}), 132.5\left(\mathrm{CH}_{2} \mathrm{CH}=\mathrm{CH}_{2}\right), 132.8(\mathrm{CH})$, $132.9(\mathrm{CH}), 133.0(\mathrm{CH}), 133.3(\mathrm{CH}), 143.6(\mathrm{C}-13), 164.6(\mathrm{CO}), 164.9(\mathrm{CO}), 165.2(\mathrm{CO}), 165.4$ (CO), 165.8 (CO), 166.2 (CO), 177.3 (C-28). Anal. Calcd for $\mathrm{C}_{85} \mathrm{H}_{92} \mathrm{O}_{18}$ : C, 72.84; H, 6.62. Found: C, $72.77 ; \mathrm{H}, 6.85$.

\subsection{Allyl 3-O-[2,3,4-tri- $O$-benzoyl- $\beta$-D-xylopyranosyl-(1 $\rightarrow$ 4)-2,3-di- $O$-benzoyl- $\alpha$-L-} arabinopyranosyl]-23-O-benzoylhederagenate (35). This compound was prepared using the general method described for 34. Reaction of allyl hederagenate $33(0.150 \mathrm{~g}, 0.24 \mathrm{mmol})$ and trichloroacetimidate $28(0.35 \mathrm{~g}, 0.37 \mathrm{mmol})$ gave $0.178 \mathrm{~g}(94 \%)$ of $\mathbf{3 5}$. $\mathrm{R}_{f}=0.61$ (toluene/acetone 9:1). $[\alpha]_{\mathrm{D}}=+46.0^{\circ}\left(c 1, \mathrm{CHCl}_{3}\right)$. As ${ }^{1} \mathrm{H}$ and ${ }^{13} \mathrm{C}$ chemical shifts for the hederagenin aglycone are nearly identical to those indicated above, only selected NMR data is presented: ${ }^{1} \mathrm{H}$ NMR $\left(\mathrm{CDCl}_{3}\right)$ : $\delta 0.69$ (s, 3H, H-24), 0.76 (s, 3H, H-26), 0.95 (s, 3H, H-29), 0.98 (s, 3H, H-30), 1.00 (s, 3H, H-25), 1.07 (s, 3H, H-27), 2.93 (dd, 1H, $J=13.6, J=3.8 \mathrm{~Hz}, \mathrm{H}-18$ ), 3.68 (dd, 1H, $J=11.6, J=4.5 \mathrm{~Hz}, \mathrm{H}-$ 3), 3.77 (brd, 1H, $J=11.0 \mathrm{~Hz}, \mathrm{H}-5$ '), 3.82 (dd, 1H, $J=12.5, J=5.2 \mathrm{~Hz}, \mathrm{H}-5$ ”), 4.02 (d, 1H, $J=11.4$ 
Hz, H-23), 4.10 (d, 1H, $J=11.6 \mathrm{~Hz}, \mathrm{H}-23), 4.34$ (dd, 1H, J= 12.4, J= 4.5 Hz, H-5'), 4.44 (m, 1H, H-4'), 4.56 (m, 3H, H-5”, $\left.\mathrm{CH}_{2} \mathrm{CH}=\mathrm{CH}_{2}\right), 4.80$ (d, 1H, J=5.9 Hz, H-1'), 5.08 (d, 1H, J=4.0 Hz, H1"), 5.25 (brd, $1 \mathrm{H}, J=10.5 \mathrm{~Hz}, \mathrm{CH}_{2} \mathrm{CH}=\mathrm{CH}_{2}$ ), 5.30 (m, 1H, H-4”), 5.36 (m, 2H, H-12, $\mathrm{CH}_{2} \mathrm{CH}=\mathrm{CH}_{2}$ ), 5.42 (dd, $1 \mathrm{H}, J=5.7, J=4.2 \mathrm{~Hz}, \mathrm{H}-2$ '), 5.45 (dd, $1 \mathrm{H}, J=8.5, J=3.2 \mathrm{~Hz}, \mathrm{H}-3$ '), 5.72 (t, 1H, $J=5.7 \mathrm{~Hz}, \mathrm{H}-3$ ”'), 5.73 (m, 1H, H-2'), 5.94 (m, 1H, $\left.\mathrm{CH}_{2} \mathrm{CH}=\mathrm{CH}_{2}\right) .{ }^{13} \mathrm{C} \mathrm{NMR}\left(\mathrm{CDCl}_{3}\right)$ :

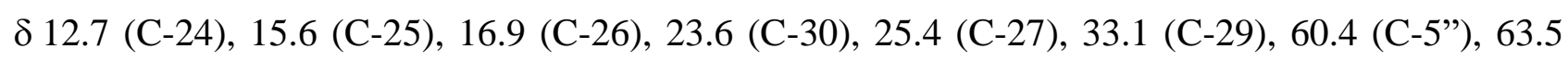
(C-5'), 65.4 (C-23), 68.5 (C-4”), 68.9 (C-3”), 69.6 (C-2”), 70.0 (C-2’), 71.3 (C-3’), 73.1 (C-4'), 82.4 (C-3), 99.8 (C-1”), 102.3 (C-1'), 122.3 (C-12), 143.6 (C-13), 177.3 (C-28). Anal. Calcd for $\mathrm{C}_{85} \mathrm{H}_{92} \mathrm{O}_{18}: \mathrm{C}, 72.84 ; \mathrm{H}, 6.62$. Found: C, 72.70; H, 6.70.

\subsection{Allyl 3-O-[2-O-benzoyl-3,4,6-tri- $O$-benzyl- $\beta$-D-glucopyranosyl-( $\rightarrow \rightarrow 3)$-2,4-di- $O$-benzoyl-} $\boldsymbol{\alpha}$-L-arabinopyranosyl]-23- $\boldsymbol{O}$-benzoylhederagenate (36). This compound was prepared using the general method described for 34. Reaction of allyl hederagenate $33(0.31 \mathrm{~g}, 0.51 \mathrm{mmol})$ and trichloroacetimidate $31(0.79 \mathrm{~g}, 0.76 \mathrm{mmol})$ gave $0.71 \mathrm{~g}(93 \%)$ of $\mathbf{3 6}$. $\mathrm{R}_{f}=0.72$ (toluene/acetone 9:1). $[\alpha]_{\mathrm{D}}=+69.0^{\circ}\left(c 1, \mathrm{CHCl}_{3}\right)$. Selected NMR data: ${ }^{1} \mathrm{H} \mathrm{NMR}\left(\mathrm{CDCl}_{3}\right): \delta 0.55(\mathrm{~s}, 3 \mathrm{H}, \mathrm{H}-24)$, 0.72 (s, 3H, H-26), 0.91 (s, 3H, H-25), 0.94 (s, 3H, H-29), 0.97 (s, 3H, H-30), 1.04 (s, 3H, H-27), $2.91(\mathrm{dd}, 1 \mathrm{H}, J=13.6, J=3.8 \mathrm{~Hz}, \mathrm{H}-18), 3.53(\mathrm{dd}, 1 \mathrm{H}, J=11.6, J=4.8 \mathrm{~Hz}, \mathrm{H}-3), 3.58$ (m, 1H, H5' '), 3.59 (dl, 1H, J= 12.7 Hz, H-5a'), 3.69 (m, 2H, H-3', H-4' '), 3.75 (m, 3H, H-23a, H-6a/b' ), $3.96(\mathrm{~d}, 1 \mathrm{H}, J=11.5 \mathrm{~Hz}, \mathrm{H}-23 \mathrm{~b}), 4.19$ (dd, 1H, $J=9.9, J=3.7 \mathrm{~Hz}, \mathrm{H}-3$ '), 4.28 (dd, 1H, $J=13.4, J=$ $\left.1.8 \mathrm{~Hz}, \mathrm{H}-5 b^{\prime}\right), 4.54$ (m, 7H, H-1', $\mathrm{CH}_{2} \mathrm{Ph}, \mathrm{CH}_{2} \mathrm{CH}=\mathrm{CH}_{2}$ ), 4.61 (d, 1H, J=11.0 Hz, $\mathrm{CH}_{2} \mathrm{Ph}$ ), 4.78 (d, 1H, J= $10.8 \mathrm{~Hz}, \mathrm{CH} 2 \mathrm{Ph}$ ), 4.79 (d, 1H, J= $7.7 \mathrm{~Hz}, \mathrm{H}-1$ '’), 5.20 (m, 1H, H-2’'), 5.23 (m, 1H, $\left.\mathrm{CH}_{2} \mathrm{CH}=\mathrm{CH}_{2}\right), 5.32(\mathrm{~m}, 1 \mathrm{H}, \mathrm{H}-12), 5.34\left(\mathrm{~m}, 1 \mathrm{H}, \mathrm{CH}_{2} \mathrm{CH}=\mathrm{CH}_{2}\right), 5.51$ (m, 1H, H-4'), 5.62 (dd, 1H, $J$ $\left.=9.8, J=7.8 \mathrm{~Hz}, \mathrm{H}-2^{\prime}\right), 5.92\left(\mathrm{~m}, 1 \mathrm{H}, \mathrm{CH}_{2} \mathrm{CH}=\mathrm{CH}_{2}\right) .{ }^{13} \mathrm{C} \mathrm{NMR}\left(\mathrm{CDCl}_{3}\right): \delta 12.5(\mathrm{C}-24), 15.5(\mathrm{C}-$ 25), 16.9 (C-26), 23.6 (C-30), 25.3 (C-27), 25.4 (C-2), 33.1 (C-29), 64.0 (C-5'), 65.3 (C-23), 69.0 (C-6’'), 71.6 (C-4'), 71.7 (C-2'), 73.4 (C-2'’), 75.1 (C-5'), 76.6 (C-3'), 77.6 (C-4'’), 82.1 (C-3), 82.7 (C-3'’), 101.4 (C-1',), 103.4 (C-1'), 122.4 (C-12), 143.6 (C-13), 177.3 (C-28). Anal. Calcd for $\mathrm{C}_{93} \mathrm{H}_{104} \mathrm{O}_{17}\left(\cdot 0.9 \mathrm{CH}_{3} \mathrm{OH}\right): \mathrm{C}, 74.07 ; \mathrm{H}, 7.12$. Found: C, 73.91; H, 7.12.

\subsection{Allyl 3-O-[2-O-benzoyl-3,4,6-tri- $O$-benzyl- $\beta$-D-glucopyranosyl-(1 $\rightarrow$ 4)-2,3-di- $O$-benzoyl-} $\boldsymbol{\alpha}$-L-arabinopyranosyl]-23- $\boldsymbol{O}$-benzoylhederagenate (37). This compound was prepared using the general method described for 34. Reaction of allyl hederagenate $33(0.11 \mathrm{~g}, 0.18 \mathrm{mmol})$ and trichloroacetimidate $32(0.28 \mathrm{~g}, 0.27 \mathrm{mmol})$ gave $0.25 \mathrm{~g}(95 \%)$ of 37 . $\mathrm{R}_{f}=0.70$ (toluene/acetone 9:1). $[\alpha]_{\mathrm{D}}=+50.0^{\circ}\left(c 1, \mathrm{CHCl}_{3}\right)$. Selected NMR data: ${ }^{1} \mathrm{H} \mathrm{NMR}\left(\mathrm{CDCl}_{3}\right): \delta 0.58(\mathrm{~s}, 3 \mathrm{H}, \mathrm{H}-24)$, 0.75 (s, 3H, H-26), 0.95 (s, 3H, H-29), 0.96 (s, 3H, H-25), 0.98 (s, 3H, H-30), 1.05 (s, 3H, H-27), $2.93(\mathrm{dd}, 1 \mathrm{H}, J=13.3, J=3.1 \mathrm{~Hz}, \mathrm{H}-18), 3.55$ (m, 1H, H-5' '), 3.63 (dd, 1H, $J=11.7, J=4.5 \mathrm{~Hz}, \mathrm{H}-$ 
3), 3.74 (m, 2H, H-6a/b') 3.76 (m, 1H, H-5a'), 3.80 (m, 2H, H-3', H-4'’), 4.01 (d, 1H, J= 11.5 Hz, H-23a), 4.07 (d, 1H, $J=11.5$ Hz, H-23b), 4.36 (dd, 1H, $J=11.8, J=6.7$ Hz, H-5b'), 4.45 (m, 1H, H-4'), 4.57 (m, 2H, $\left.\mathrm{CH}_{2} \mathrm{CH}=\mathrm{CH}_{2}\right), 4.60$ (d, $\left.1 \mathrm{H}, J=12.3 \mathrm{~Hz}, \mathrm{CH}_{2} \mathrm{Ph}\right), 4.62(\mathrm{~d}, 1 \mathrm{H}, J=10.9 \mathrm{~Hz}$, $\left.\mathrm{CH}_{2} \mathrm{Ph}\right), 4.65\left(\mathrm{~d}, 1 \mathrm{H}, J=9.0 \mathrm{~Hz}, \mathrm{CH}_{2} \mathrm{Ph}\right), 4.67\left(\mathrm{~d}, 1 \mathrm{H}, J=12.0 \mathrm{~Hz}, \mathrm{CH}_{2} \mathrm{Ph}\right), 4.73(\mathrm{~d}, 1 \mathrm{H}, J=11.0$ $\mathrm{Hz}, \mathrm{CH}_{2} \mathrm{Ph}$ ), 4.80 (d, 1H, J=7.5 Hz, H-1'’), 4.80 (m, 1H, H-1'), 4.85 (d, 1H, J=10.9 Hz, CH $\mathrm{H}_{2} \mathrm{Ph}$ ), $5.25\left(\mathrm{~m}, 1 \mathrm{H}, \mathrm{CH}_{2} \mathrm{CH}=\mathrm{CH}_{2}\right), 5.35(\mathrm{~m}, 2 \mathrm{H}, \mathrm{H}-12, \mathrm{H}-2$ ') $), 5.36\left(\mathrm{~m}, 1 \mathrm{H}, \mathrm{CH}_{2} \mathrm{CH}=\mathrm{CH}_{2}\right), 5.38(\mathrm{~m}, 1 \mathrm{H}$, H-3'), $5.53\left(\mathrm{dd}, 1 \mathrm{H}, J=6.7, J=4.4 \mathrm{~Hz}, \mathrm{H}-2\right.$ ') $5.95\left(\mathrm{~m}, 1 \mathrm{H}, \mathrm{CH}_{2} \mathrm{CH}=\mathrm{CH}_{2}\right) .{ }^{13} \mathrm{C} \mathrm{NMR}\left(\mathrm{CDCl}_{3}\right): \delta$ 12.6 (C-24), 15.6 (C-25), 16.9 (C-26), 23.6 (C-30), 25.2 (C-2), 25.4 (C-27), 33.1 (C-29), 62.0 (C5'), 65.5 (C-23), 68.6 (C-6’'), 69.8 (C-2', C-3'), 71.4 (C-4'), 73.5 (C-2'’), 75.2 (C-5'’), 77.7 (C4’”), 82.2 (C-3), 82.7 (C-3’'), 100.2 (C-1'”), 101.0 (C-1'), 122.4 (C-12), 143.6 (C-13), 177.3 (C-28). Anal. Calcd for $\mathrm{C}_{93} \mathrm{H}_{104} \mathrm{O}_{17}$ : C, 74.78; H, 7.02. Found: C, 74.51; H, 7.29.

\subsection{Allyl 3- $O$-[2,3,4-tri- $O$-benzoyl- $\beta$-D-xylopyranosyl-(1 $\rightarrow 2)-2,3$-di- $O$-benzoyl- $\beta$-L-} arabinopyranosyl]-23- $O$-benzoylhederagenate (38). This compound was prepared at $0{ }^{\circ} \mathrm{C}$ using the general method described for 34. Reaction of allyl hederagenate $33(0.26 \mathrm{~g}, 0.41 \mathrm{mmol})$ and trichloroacetimidate $27(0.59 \mathrm{~g}, 0.62 \mathrm{mmol})$ gave $0.55 \mathrm{~g}(94 \%)$ of 38. $\mathrm{R}_{f}=0.62$ (toluene/acetone 9:1). $[\alpha]_{\mathrm{D}}=+81.9^{\circ}\left(c 1, \mathrm{CHCl}_{3}\right)$. Selected NMR data: ${ }^{1} \mathrm{H} \mathrm{NMR}\left(\mathrm{CDCl}_{3}\right): \delta 0.83(\mathrm{~s}, 3 \mathrm{H}, \mathrm{H}-26), 0.96$ (s, 6H, H-24, H-29), 0.99 (s, 3H, H-30), 1.10 (s, 3H, H-25), 1.13 (s, 3H, H-27), 2.96 (dd, 1H, J= 14.2, $J=3.7 \mathrm{~Hz}, \mathrm{H}-18$ ), 3.72 (dd, $1 \mathrm{H}, J=11.9, J=8.3 \mathrm{~Hz}, \mathrm{H}-5$ '), 3.82 (brd, 1H, $J=13.0 \mathrm{~Hz}, \mathrm{H}-5^{\prime}$ ), $3.88(\mathrm{dd}, 1 \mathrm{H}, J=11.7, J=4.3 \mathrm{~Hz}, \mathrm{H}-3), 4.20$ (brd, 1H, $J=12.7 \mathrm{~Hz}, \mathrm{H}-5$ '), 4.33 (m, 2H, H-23), 4.39 (dd, 1H, $J=10.4, J=3.5 \mathrm{~Hz}, \mathrm{H}-2$ ') 4.49 (dd, 1H, $J=11.9, J=4.8 \mathrm{~Hz}, \mathrm{H}-5$ ”), 4.60 (m, 2H, $\left.\mathrm{CH}_{2} \mathrm{CH}=\mathrm{CH}_{2}\right), 5.06\left(\mathrm{~d}, 1 \mathrm{H}, J=6.2 \mathrm{~Hz}, \mathrm{H}-1\right.$ '), $5.28\left(\mathrm{dd}, 1 \mathrm{H}, J=10.5, J=1.0 \mathrm{~Hz}, \mathrm{CH}_{2} \mathrm{CH}_{=} \mathrm{CH}_{2}\right)$, 5.39 (m, 6H, H-1', H-2”, H-4”, H-12, $\mathrm{CH}_{2} \mathrm{CH}=\mathrm{CH}_{2}$ ), 5.62 (dd, $1 \mathrm{H}, J=10.5, J=3.5 \mathrm{~Hz}, \mathrm{H}-3$ '), 5.69 (m, 1H, H-4'), 5.74 (t, 1H, $J=8.4 \mathrm{~Hz}, \mathrm{H}-3$ ”), 5.97 (m, 1H, $\left.\mathrm{CH}_{2} \mathrm{CH}=\mathrm{CH}_{2}\right) .{ }^{13} \mathrm{C}^{\mathrm{NMR}}\left(\mathrm{CDCl}_{3}\right)$ : $\delta 13.1$ (C-24), 15.8 (C-25), 17.0 (C-26), 21.8 (C-2), 23.6 (C-30), 25.5 (C-27), 33.1 (C-29), 61.0 (C5’), 62.2 (C-5”), 66.0 (C-23), 69.6 (C-3’), 69.7 (C-4”), 70.2 (C-4'), 70.8 (C-2”), 71.1 (C-3”), 74.8 (C-2'), 78.8 (C-3), 96.7 (C-1'), 102.0 (C-1”), 122.4 (C-12), 143.6 (C-13), 177.3 (C-28). Anal. Calcd for $\mathrm{C}_{85} \mathrm{H}_{92} \mathrm{O}_{18}$ : C, 72.84; H, 6.62. Found: C, 72.45; H, 6.43.

\subsection{Allyl 3-O-[2-O-benzoyl-3,4,6-tri- $O$-benzyl- $\beta$-D-glucopyranosyl-(1 $\rightarrow 2$ )-3,4-di- $O$-benzoyl-} $\beta$-L-arabinopyranosyl]-23- $\boldsymbol{O}$-benzoylhederagenate (39). This compound was prepared at $0{ }^{\circ} \mathrm{C}$ using the general method described for 34. Reaction of allyl hederagenate $33(0.40 \mathrm{~g}, 0.65 \mathrm{mmol})$ and trichloroacetimidate $30(1.01 \mathrm{~g}, 0.97 \mathrm{mmol})$ gave $0.82 \mathrm{~g}(85 \%)$ of $39 . \mathrm{R}_{f}=0.75$ (toluene/acetone 9:1). $[\alpha]_{\mathrm{D}}=+83.3^{\circ}\left(c 1, \mathrm{CHCl}_{3}\right)$. Selected NMR data: ${ }^{1} \mathrm{H} \mathrm{NMR}\left(\mathrm{CDCl}_{3}\right): \delta 0.81$ (s, 3H, H-26), 0.94 (s, 3H, H-24), 0.97 (s, 3H, H-29), 1.01 (s, 3H, H-30), 1.04 (s, 3H, H-25), 1.12 (s, 
3H, H-27), 2.96 (dd, 1H, $J=13.4, J=3.3 \mathrm{~Hz}, \mathrm{H}-18), 3.65$ (m, 1H, H-5' '), 3.76 (m, 1H, H-3), 3.77 (t, 1H, $J=9.2$ Hz, H-3' ') 3.82 (m, 1H, H-5a'), 3.84 (m, 2H, H-6a/b' '), 3.89 (t, 1H, J=9.2 Hz, H4' '), 4.23 (dl, 1H, J=12.6 Hz, H-5b'), 4.27 (d, 1H, J=11.5 Hz, H-23a), 4.31 (d, 1H, J= $11.5 \mathrm{~Hz}$, H-23b), 4.37 (dd, 1H, $J=10.5, J=3.6 \mathrm{~Hz}, \mathrm{H}-2$ '), 4.56 (d, 1H, $\left.J=12.0 \mathrm{~Hz}, \mathrm{CH}_{2} \mathrm{Ph}\right), 4.58$ (d, 1H, $J=$ $\left.10.9 \mathrm{~Hz}, \mathrm{CH}_{2} \mathrm{Ph}\right), 4.61\left(\mathrm{~m}, 2 \mathrm{H}, \mathrm{CH}_{2} \mathrm{CH}=\mathrm{CH}_{2}\right), 4.65\left(\mathrm{~d}, 1 \mathrm{H}, J=11.0 \mathrm{~Hz}, \mathrm{CH}_{2} \mathrm{Ph}\right), 4.68(\mathrm{~d}, 2 \mathrm{H}, J=$ $11.5 \mathrm{~Hz}, \mathrm{CH}_{2} \mathrm{Ph}$ ), 4.85 (d, 1H, J=7.8 Hz, H-1' '), 4.87 (d, 1H, J=10.8 Hz, CH${ }_{2} \mathrm{Ph}$ ), 5.29 (m, 2H, H2' ', $\mathrm{CH}_{2} \mathrm{CH}=\mathrm{CH}_{2}$ ), 5.39 (m, 2H, H-1', $\mathrm{CH}_{2} \mathrm{CH}=\mathrm{CH}_{2}$ ), 5.53 (dd, $1 \mathrm{H}, J=10.5, J=3.5 \mathrm{~Hz}, \mathrm{H}-3$ '), 5.67 (m, 1H, H-4'), $5.97\left(\mathrm{~m}, 1 \mathrm{H}, \mathrm{CH}_{2} \mathrm{CH}=\mathrm{CH}_{2}\right) .{ }^{13} \mathrm{C} \mathrm{NMR}\left(\mathrm{CDCl}_{3}\right): \delta 13.1$ (C-24), 15.7 (C-25), 17.0 (C-26), 22.0 (C-2), 23.6 (C-30), 25.4 (C-27), 33.1 (C-29), 61.0 (C-5'), 66.3 (C-23), 68.8 (C-6’'), 70.0 (C-3’), 70.3 (C-4'), 73.6 (C-2'), 73.7 (C-2’’), 75.1 (C-5’’), 77.8 (C-4’’), 79.6 (C-3), 82.7 (C3'”), 97.4 (C-1'), 101.6 (C-1'”), 122.5 (C-12), 143.5 (C-13), 177.3 (C-28). Anal. Calcd for $\mathrm{C}_{93} \mathrm{H}_{104} \mathrm{O}_{17}$ : C, 74.78; H, 7.02. Found: C, 74.44; H, 7.20.

\subsection{Allyl 3- $O$-[2,3,4-tri- $O$-benzoyl- $\beta$-D-xylopyranosyl-(1 $\rightarrow 2)-2,3$-di- $O$-benzoyl- $\alpha$-L-}

arabinopyranosyl]-23-O-benzoylhederagenate (40). Allyl hederagenate 33 (0.8 g, $1.3 \mathrm{mmol})$, trichloroacetimidate $27(2.46 \mathrm{~g}, 2.6 \mathrm{mmol}, 2.0 \mathrm{eq})$ and $4 \AA$ powdered molecular sieves $(7 \mathrm{~g})$ were stirred for $1 \mathrm{~h}$ at room temperature in dry acetonitrile $(20 \mathrm{~mL})$. The mixture was cooled to $-35{ }^{\circ} \mathrm{C}$ for 30 minutes followed by the rapid addition of a $0.1 \mathrm{M}$ solution of TMSOTf in acetonitrile $(3.9 \mathrm{~mL}$, $0.39 \mathrm{mmol}, 0.3 \mathrm{eq})$. The reaction was stirred at this temperature until tlc indicated the disappearance of the allyl hederagenin. Triethylamine was added and the mixture was filtered through Celite and evaporated. The crude residue was purified by column chromatography (toluene/acetone 99:1) to give $0.68 \mathrm{~g}(38 \%)$ of the desired $\alpha$ anomer, and $0.48 \mathrm{~g}$ of a mixture of anomeric products. HPLC separation (100\% acetonitrile) gave a further $0.36 \mathrm{~g}(20 \%)$ of the desired saponin 40 as a white foam (total yield 58\%), and $0.12 \mathrm{~g} \mathrm{(6 \% )} \mathrm{of} \mathrm{the} \beta$ anomer 38 which was previously described above. $\mathrm{R} f=0.63$ (toluene/acetone 9:1). $[\alpha]_{\mathrm{D}}=+47.1^{\circ}\left(\right.$ c $\left.1, \mathrm{CHCl}_{3}\right)$. Selected NMR data: ${ }^{1} \mathrm{H}$ NMR $\left(\mathrm{CDCl}_{3}\right): \delta 0.77$ (s, 3H, H-24), 0.79 (s, 3H, H-26), 0.96 (s, 3H, H-29), 0.98 (s, 3H, H-30), 1.02 (s, $3 \mathrm{H}, \mathrm{H}-25), 1.13$ (s, 3H, H-27), 2.94 (dd, 1H, $J=13.7, J=3.8 \mathrm{~Hz}, \mathrm{H}-18$ ), 3.66 (dd, 1H, $J=12.0, J=$ $7.8 \mathrm{~Hz}, \mathrm{H}-5$ ”), 3.73 (dd, 1H, $J=11.6, J=4.4 \mathrm{~Hz}, \mathrm{H}-3$ ), 3.84 (dd, 1H, $J=11.6, J=3.2 \mathrm{~Hz}, \mathrm{H}-5$ '), 4.20 (d, 1H, $J=11.4 \mathrm{~Hz}, \mathrm{H}-23), 4.27$ (dd, 1H, $J=11.7, J=7.1 \mathrm{~Hz}, \mathrm{H}-5$ '), 4.34 (dd, 1H, $J=6.0, J=$ $3.9 \mathrm{~Hz}, \mathrm{H}-2$ '), 4.37 (m, 1H, H-23), 4.41 (dd, 1H, $J=12.1, J=4.4 \mathrm{~Hz}, \mathrm{H}-5$ '), 4.58 (m, 2H, $\left.\mathrm{CH}_{2} \mathrm{CH}=\mathrm{CH}_{2}\right), 4.89$ (d, 1H, $J=3.5 \mathrm{~Hz}, \mathrm{H}-1^{\prime}$ ), 5.14 (d, 1H, J=5.9 Hz, H-1”), 5.26 (d, 1H, J= 10.5 $\mathrm{Hz}, \mathrm{CH}_{2} \mathrm{CH}=\mathrm{CH}_{2}$ ), 5.32 (m, 1H, H-4”), 5.37 (m, 3H, H-12, H-3', $\left.\mathrm{CH}_{2} \mathrm{CH}=\mathrm{CH}_{2}\right), 5.44$ (dd, 1H, $J=$ $7.7, J=6.1 \mathrm{~Hz}, \mathrm{H}-2 ”), 5.48\left(\mathrm{~m}, 1 \mathrm{H}, \mathrm{H}-4^{\prime}\right), 5.74$ (t, 1H, $\left.J=7.8 \mathrm{~Hz}, \mathrm{H}-3 ”\right), 5.95$ (m, 1H, $\left.\mathrm{CH}_{2} \mathrm{CH}=\mathrm{CH}_{2}\right) .{ }^{13} \mathrm{C} \mathrm{NMR}\left(\mathrm{CDCl}_{3}\right): \delta 12.7(\mathrm{C}-24), 15.8(\mathrm{C}-25), 17.0(\mathrm{C}-26), 23.6(\mathrm{C}-30), 25.2(\mathrm{C}-$ 2), 25.4 (C-27), 33.1 (C-29), 59.4 (C-5'), 61.7 (C-5”), 65.7 (C-23), 67.1 (C-4'), 69.2 (C-4”), 70.7 
(C-3”, C-2”, C-3'), 75.1 (C-2'), 82.9 (C-3), 101.0 (C-1”), 101.9 (C-1'), 122.3 (C-12), 143.7 (C-13), 177.3 (C-28). Anal. Calcd for $\mathrm{C}_{85} \mathrm{H}_{92} \mathrm{O}_{18}$ : C, 72.84; H, 6.62. Found: C, 72.69; H, 6.84.

\subsection{Allyl 3-O-[2-O-benzoyl-3,4,6-tri- $O$-benzyl- $\beta$-D-glucopyranosyl-(1 $\rightarrow 2$ )-3,4-di- $O$-benzoyl-} $\boldsymbol{\alpha}$-L-arabinopyranosyl]-23- $\boldsymbol{O}$-benzoylhederagenate (41). This product was prepared in acetonitrile as described for compound 40. Reaction of allyl hederagenate 33 ( $0.83 \mathrm{~g}, 1.3 \mathrm{mmol})$ and trichloroacetimidate $30(2.8 \mathrm{~g}, 2.7 \mathrm{mmol})$ gave $1.22 \mathrm{~g}(61 \%)$ of $\mathbf{4 1}$ as well as $0.28 \mathrm{~g}(14 \%)$ of the $\beta$ coupling product 39. $\mathrm{R}_{f}=0.69$ (toluene/acetone 9:1). $[\alpha]_{\mathrm{D}}=+47.5^{\circ}\left(c 1, \mathrm{CHCl}_{3}\right) .{ }^{1} \mathrm{H} \mathrm{NMR}$ $\left(\mathrm{CDCl}_{3}\right): \delta 0.73$ (s, 3H, H-24), 0.76 (s, 3H, H-26), 0.95 (s, 3H, H-29), 0.97 (s, 3H, H-25), 0.98 (s, $3 \mathrm{H}, \mathrm{H}-30$ ), 1.07 (s, 3H, H-27), 2.92 (dd, 1H, $J=13.7, J=3.8 \mathrm{~Hz}, \mathrm{H}-18), 3.64$ (dt, $J=9.7, J=3.3$ Hz, H-5' '), 3.69 (dd, 1H, J=11.7, J=4.6 Hz, H-3), 3.75 (t, 1H, J=9.2 Hz, H-3' '), 3.78 (m, 1H, H5a'), 3.80 (m, 2H, H-6a/b' '), 3.85 (t, 1H, J=9.2 Hz, H-4' '), 4.20 (dd, 1H, J=12.0, J=6.4 Hz, H5b'), 4.27 (d, 1H, J = $11.4 \mathrm{~Hz}, \mathrm{H}-23 \mathrm{a}), 4.32$ (m, 2H, H-2', H-23b), 4.57 (m, 4H, $\mathrm{CH}_{2} \mathrm{CH}=\mathrm{CH}_{2}$, $\left.\mathrm{CH}_{2} \mathrm{Ph}\right), 4.60\left(\mathrm{~m}, 2 \mathrm{H}, \mathrm{CH}_{2} \mathrm{Ph}\right), 4.69\left(\mathrm{~d}, 1 \mathrm{H}, J=11.2 \mathrm{~Hz}, \mathrm{CH}_{2} \mathrm{Ph}\right), 4.82\left(\mathrm{~m}, 1 \mathrm{H}, \mathrm{H}-1^{\prime}\right), 4.83(\mathrm{~d}, 1 \mathrm{H}, J$ $\left.=10.8 \mathrm{~Hz}, \mathrm{CH}_{2} \mathrm{Ph}\right), 4.89$ (d, 1H, J=8.0 Hz, H-1' '), 5.25 (m, 2H, H-3', $\left.\mathrm{CH}_{2} \mathrm{CH}=\mathrm{CH}_{2}\right), 5.31(\mathrm{dd}, 1 \mathrm{H}$, $J=9.4, J=8.1 \mathrm{~Hz}, \mathrm{H}-2$ ' ') 4.35 (m, 1H, H-12), 4.36 (m, 1H, $\left.\mathrm{CH}_{2} \mathrm{CH}=\mathrm{CH}_{2}\right), 4.38$ (m, 1H, H-4'), $5.95\left(\mathrm{~m}, 1 \mathrm{H}, \mathrm{CH}_{2} \mathrm{CH}=\mathrm{CH}_{2}\right) .{ }^{13} \mathrm{C} \mathrm{NMR}\left(\mathrm{CDCl}_{3}\right): \delta 12.8(\mathrm{C}-24), 15.6(\mathrm{C}-25), 17.0(\mathrm{C}-26), 23.6(\mathrm{C}-$ 30), 25.1 (C-2), 25.4 (C-27), 33.1 (C-29), 60.2 (C-5'), 65.9 (C-23), 67.4 (C-4'), 69.1 (C-6’'), 71.8 (C-3'), 73.4 (C-2'’), 74.7 (C-2'), 75.5 (C-5'’), 77.8 (C-4'’), 82.7 (C-3'’), 82.8 (C-3), 101.4 (C-1'’), 101.9 (C-1'), 122.3 (C-12), 143.7 (C-13), 177.3 (C-28). Anal. Calcd for $\mathrm{C}_{93} \mathrm{H}_{104} \mathrm{O}_{17}$ : C, 74.78; H, 7.02. Found: C, 74.44; H, 7.31.

\subsection{3- $O$-[ $\beta$-D-xylopyranosyl-( $(\rightarrow 2)$ - $\alpha$-L-arabinopyranosyl]hederagenin $(1)$. General method}

1. In a typical experiment, $\mathrm{Pd}\left(\mathrm{PPh}_{3}\right)_{4}(0.57 \mathrm{~g}, 0.49 \mathrm{mmol}, 1.0 \mathrm{eq})$ was added to a solution of saponin $40(0.680 \mathrm{~g}, 0.49 \mathrm{mmol})$ in $3 \% \mathrm{KOH}$ in $\mathrm{MeOH}(39 \mathrm{~mL})$. After heating to $60{ }^{\circ} \mathrm{C}$ for $6 \mathrm{~h}$, the reaction was neutralized with Amberlite IR $120\left(\mathrm{H}^{+}\right.$form), filtered and evaporated. The crude residue was purified by column chromatography $\left(\mathrm{CH}_{2} \mathrm{Cl}_{2} / \mathrm{MeOH} 9: 1\right.$ to $\left.8: 2\right)$ to give $0.280 \mathrm{~g}(76 \%)$ of the deprotected saponin $\mathbf{1}$ as an amorphous solid.

General method 2. Use of a catalytic amount of $\mathrm{Pd}\left(\mathrm{PPh}_{3}\right)_{4}$ : in a typical experiment $\mathrm{Pd}\left(\mathrm{PPh}_{3}\right)_{4}$ $(0.025 \mathrm{~g}, 0.02 \mathrm{mmol}, 0.3 \mathrm{eq})$ was added to a solution of saponin $40(0.102 \mathrm{~g}, 0.07 \mathrm{mmol})$ in a $\mathrm{THF} / 3 \% \mathrm{KOH}$ in $\mathrm{MeOH}(1: 1)$ mixture $(6 \mathrm{~mL})$. After heating to $60{ }^{\circ} \mathrm{C}$ for $7 \mathrm{~h}$, the reaction was neutralized with Amberlite IR $120\left(\mathrm{H}^{+}\right.$form), filtered and evaporated. The crude residue was purified by column chromatography $\left(\mathrm{CH}_{2} \mathrm{Cl}_{2} / \mathrm{MeOH} 9: 1\right.$ to $\left.8: 2\right)$ to give $0.048 \mathrm{~g}(89 \%)$ of the deprotected saponin $\mathbf{1}$ as an amorphous solid. 
$[\alpha]_{\mathrm{D}}=+41.0^{\circ}\left(c 0.5\right.$, pyridine). ${ }^{1} \mathrm{H}$ NMR (pyridine- $\left.d_{5}\right): \delta 0.90(\mathrm{~s}, 3 \mathrm{H}, \mathrm{H}-29), 0.93(\mathrm{~s}, 3 \mathrm{H}, \mathrm{H}-25)$, 0.98 (s, 3H, H-30), 1.00 (s, 6H, H-24, H-26), 1.03-2.22 (m, 22H, H-1, H-2, H-5, H-6, H-7, H-9, H11, H-15, H-16, H-19, H-21, H-22), 1.22 (s, 3H, H-27), 3.26 (dd, 1H, J= 13.6, J= 3.8 Hz, H-18), 3.56 (m, 1H, H-5”), 3.64 (brd, 1H, $J=11.1 \mathrm{~Hz}, \mathrm{H}-5$ '), 3.68 (d, 1H, J=11.0 Hz, H-23), 4.08 (m, 1H, H-2”), 4.10 (m, 1H, H-3”), 4.15 (dd, 1H, J= 8.4, J= 2.3 Hz, H-3’), 4.18 (m, 1H, H-4”), 4.24 (m, 1H, H-5'), 4.25 (m, 1H, H-4'), 4.27 (m, 1H, H-3), 4.31 (dd, 1H, J= 11.4, J= 5.2 Hz, H-5’), 4.35 (d, $1 \mathrm{H}, J=11.1 \mathrm{~Hz}, \mathrm{H}-23), 4.53$ (brt, 1H, $J=7.5 \mathrm{~Hz}, \mathrm{H}-2$ '), 5.07 (d, 1H, J=6.5 Hz, H-1”), 5.11 (d, 1H, $J=6.6 \mathrm{~Hz}, \mathrm{H}-1$ '), 5.46 (m, 1H, H-12). ${ }^{13} \mathrm{C}$ NMR (pyridine- $\left.d_{5}\right): \delta 12.9$ (C-24), 15.8 (C-25), 17.1 (C26), 17.8 (C-6), 23.3 (C-16), 23.4 (C-30), 23.5 (C-11), 25.9 (C-27), 26.0 (C-2), 28.0 (C-15), 30.6 (C-20), 32.5 (C-7), 32.9 (C-22, C-29), 33.8 (C-21), 36.6 (C-10), 38.5 (C-1), 39.4 (C-8), 41.6 (C-18), 41.8 (C-14), 43.3 (C-4), 46.1 (C-19), 46.3 (C-17), 47.0 (C-5), 47.8 (C-9), 63.2 (C-23), 65.6 (C-5'), 67.1 (C-5”), 68.4 (C-4'), 70.6 (C-4”), 73.6 (C-3’), 75.8 (C-2”), 77.9 (C-3”), 81.1 (C-3), 81.5 (C-2'), 104.2 (C-1'), 106.4 (C-1'), 122.3 (C-12), 144.5 (C-13), 179.9 (C-28). HRMS: $\mathrm{C}_{40} \mathrm{H}_{64} \mathrm{O}_{12} \mathrm{Na}$ calcd 759.4295 ; found 759.4316 .

\subsection{3- $O$-[ $\beta$-D-xylopyranosyl- $(1 \rightarrow 2)-\beta$-L-arabinopyranosyl]hederagenin $\quad(2)$. Using a} stoichiometric amount of $\mathrm{Pd}\left(\mathrm{PPh}_{3}\right)_{4}$ in the general deprotection method 1 described for $\mathbf{1}$, the deprotection of saponin $38(0.52 \mathrm{~g}, 0.37 \mathrm{mmol})$ gave $0.17 \mathrm{~g}(64 \%)$ of $\mathbf{2}$. Use of the catalytic method with $0.115 \mathrm{~g}(0.08 \mathrm{mmol})$ of $\mathbf{3 8}$ gave $0.050 \mathrm{~g}(84 \%)$ of $\mathbf{2}$. $[\alpha]_{\mathrm{D}}=+82.2^{\circ}(c 0.5$, pyridine $)$. Selected NMR data: ${ }^{1} \mathrm{H}$ NMR (pyridine- $d_{5}$ ) : $\delta 0.85$ (s, 3H, H-24), 0.85 (s, 3H, H-25), 0.92 (s, 3H, H-29), 0.99 (s, 3H, H-30), 1.00 (s, 3H, H-26), 1.24 (s, 3H, H-27), 3.27 (dd, 1H, J= 13.5, J= 3.6 Hz, H-18), 3.50 (dd, 1H, $J=11.0, J=10.1 \mathrm{~Hz}, \mathrm{H}-5$ ”), 3.70 (d, 1H, $J=10.7 \mathrm{~Hz}, \mathrm{H}-23$ ), 3.93 (d, 1H, $J=10.9 \mathrm{~Hz}$, H-23), 4.10 (dd, 1H, $\left.J=8.7, J=7.0 \mathrm{~Hz}, \mathrm{H}-2^{\prime \prime}\right), 4.12$ (dd, 1H, $J=10.5, J=1.7 \mathrm{~Hz}, \mathrm{H}-5$ '; t, $1 \mathrm{H}, J=$ 8.6 Hz, H-3”), 4.18 (m, 1H, H-4”), 4.21 (dd, 1H, $J=11.9, J=4.8$ Hz, H-3), 4.25 (dd, 1H, $J=11.3, J$ $=5.1 \mathrm{~Hz}, \mathrm{H}-5^{\prime \prime}$ ), 4.43 (m, 1H, H-4'), 4.44 (brd, 1H, $J=11.3 \mathrm{~Hz}, \mathrm{H}-5$ '), 4.62 (dd, 1H, $J=9.9, J=3.1$ Hz, H-3'), 4.67 (dd, 1H, $J=9.9, J=3.2$ Hz, H-2'), 5.00 (d, 1H, J= 7.1 Hz, H-1”), 5.48 (m, 1H, H12), $5.69\left(\mathrm{~d}, 1 \mathrm{H}, J=3.3 \mathrm{~Hz}, \mathrm{H}-1\right.$ ') ${ }^{13} \mathrm{C}$ NMR (pyridine-d5) : $\delta 13.8(\mathrm{C}-24), 15.6(\mathrm{C}-25), 17.2(\mathrm{C}-$ 26), 22.2 (C-2), 23.5 (C-30), 25.9 (C-27), 33.0 (C-29), 64.0 (C-23), 64.3 (C-5'), 66.6 (C-5”), 69.2 (C-3'), 70.0 (C-4'), 70.5 (C-4”), 74.8 (C-2”), 77.4 (C-3), 77.5 (C-3”), 79.4 (C-2'), 97.6 (C-1'), 106.6 (C-1”), 122.3 (C-12), 144.6 (C-13), 180.3 (C-28). HRMS: $\mathrm{C}_{40} \mathrm{H}_{64} \mathrm{O}_{12} \mathrm{Na}$ calcd 759.4295; found 759.4275 .

\subsection{3- $O$-[ $\beta$-D-xylopyranosyl-(1 $\rightarrow 3)$ - $\alpha$-L-arabinopyranosyl]hederagenin $\quad(3)$. Using a} stoichiometric amount of $\mathrm{Pd}\left(\mathrm{PPh}_{3}\right)_{4}$ in the general deprotection method 1 described for $\mathbf{1}$, the deprotection of saponin $34(0.26 \mathrm{~g}, 0.18 \mathrm{mmol})$ gave $0.11 \mathrm{~g}(82 \%)$ of $\mathbf{3}$. Use of the catalytic method 
with $0.055 \mathrm{~g}(0.04 \mathrm{mmol})$ of $\mathbf{3 4}$ gave $0.021 \mathrm{~g}(72 \%)$ of $\mathbf{3}$. $[\alpha]_{\mathrm{D}}=+47.6^{\circ}(c 0.5$, pyridine $)$. Selected NMR data: ${ }^{1} \mathrm{H}$ NMR (pyridine-d5) : $\delta 0.91$ (s, 3H, H-25), 0.92 (s, 3H, H-24), 0.92 (s, 3H, H-29), 0.98 (s, 3H, H-30), 1.00 (s, 3H, H-26), 1.24 (s, 3H, H-27), 3.27 (dd, 1H, J= 13.7, J= 3.7 Hz, H-18), 3.70 (d, 1H, $J=10.7 \mathrm{~Hz}, \mathrm{H}-23), 3.71$ (m, 1H, H-5”), 3.75 (brd, 1H, J = $11.6 \mathrm{~Hz}, \mathrm{H}-5$ '), 4.03 (dd, $1 \mathrm{H}, J=8.8, J=7.7 \mathrm{~Hz}, \mathrm{H}-2 ”), 4.10$ (dd, 1H, $J=9.4, J=3.3 \mathrm{~Hz}, \mathrm{H}-3$ '), 4.18 (m, 2H, H-3”, H-4”), $4.26(\mathrm{dd}, 1 \mathrm{H}, J=12.0, J=2.0 \mathrm{~Hz}, \mathrm{H}-5$ ') 4.29 (dd, 1H, $J=12.2, J=4.6 \mathrm{~Hz}, \mathrm{H}-3), 4.32(\mathrm{~d}, 1 \mathrm{H}, J=$ 10.6 Hz, H-23), 4.33 (dd, 1H, $J=10.7, J=5.2$ Hz, H-5”), 4.39 (m, 1H, H-4'), 4.59 (dd, 1H, J= 9.2, $J=7.7 \mathrm{~Hz}, \mathrm{H}-2$ ') 5.04 (d, 1H, $J=7.5 \mathrm{~Hz}, \mathrm{H}-1$ '), 5.20 (d, 1H, J=7.6 Hz, H-1'), 5.47 (m, 1H, H-12).

${ }^{13} \mathrm{C}$ NMR (pyridine- $d_{5}$ ) : $\delta 13.4$ (C-24), 15.8 (C-25), 17.2 (C-26), 23.5 (C-30), 25.9 (C-27), 33.0 (C29), 63.6 (C-23), 66.7 (C-5’), 66.8 (C-5”), 69.0 (C-4'), 70.6 (C-4”), 71.5 (C-2'), 74.9 (C-2”), 77.5 (C-3”), 81.5 (C-3), 83.3 (C-3'), 106.2 (C-1'), 106.3 (C-1”), 122.3 (C-12), 144.5 (C-13), 180.2 (C28). HRMS: $\mathrm{C}_{40} \mathrm{H}_{64} \mathrm{O}_{12} \mathrm{Na}$ calcd 759.4295; found 759.4303 .

\subsection{3- $O$-[ $\beta$-D-xylopyranosyl-(1 $\rightarrow 4)$ - $\alpha$-L-arabinopyranosyl]hederagenin $\quad$ (4). Using a} stoichiometric amount of $\mathrm{Pd}\left(\mathrm{PPh}_{3}\right)_{4}$ in the general deprotection method 1 described for $\mathbf{1}$, the deprotection of saponin $35(0.32 \mathrm{~g}, 0.23 \mathrm{mmol})$ gave $0.14 \mathrm{~g}(84 \%)$ of 4 . Use of the catalytic method with $0.108 \mathrm{~g}(0.08 \mathrm{mmol})$ of 35 gave $0.042 \mathrm{~g}(74 \%)$ of 4 . $[\alpha]_{\mathrm{D}}=+46.8^{\circ}(c 0.5$, pyridine $)$. Selected NMR data: ${ }^{1} \mathrm{H}$ NMR (pyridine- $\left.d_{5}\right): \delta 0.88$ (s, 3H, H-24), 0.92 (s, 6H, H-25, H-29), 0.98 (s, 3H, H30), 0.99 (s, 3H, H-26), 1.23 (s, 3H, H-27), 3.27 (dd, 1H, J= 13.7, J= 3.8 Hz, H-18), 3.64 (brt, 1H, $J=10.6 \mathrm{~Hz}, \mathrm{H}-5$ ') 3.67 (d, 1H, $J=10.7$ Hz, H-23), 3.76 (brd, 1H, $J=11.6 \mathrm{~Hz}, \mathrm{H}-5$ '), 4.02 (dd, 1H, $J=8.7, J=7.9 \mathrm{~Hz}, \mathrm{H}-2$ ”), 4.13 (t, 1H, $J=8.8 \mathrm{~Hz}, \mathrm{H}-3$ ”), 4.15 (dd, 1H, $J=9.3, J=3.6 \mathrm{~Hz}, \mathrm{H}-3$ '), 4.20 (m, 1H, H-4”), 4.23 (dd, 1H, $J=12.2, J=4.6$ Hz, H-3), 4.26 (brd, 1H, $J=11.1$ Hz, H-23), 4.27 (m, 1H, H-4'), 4.30 (dd, 1H, $J=11.3, J=5.2 \mathrm{~Hz}, \mathrm{H}-5$ '), 4.40 (dd, 1H, $J=9.1, J=7.4 \mathrm{~Hz}, \mathrm{H}-2^{\prime}$ ), $4.43(\mathrm{dd}, 1 \mathrm{H}, J=12.5, J=2.2 \mathrm{~Hz}, \mathrm{H}-5$ '), 4.96 (d, 1H, J=7.3 Hz, H-1'), 5.06 (d, 1H, J= 7.7 Hz, H1 ”), 5.47 (m, 1H, H-12). ${ }^{13} \mathrm{C}$ NMR (pyridine- $\left.d_{5}\right): \delta 13.3$ (C-24), 15.8 (C-25), 17.2 (C-26), 23.5 (C30), 25.9 (C-27), 33.0 (C-29), 63.9 (C-23), 66.0 (C-5'), 66.9 (C-5”), 70.5 (C-4”), 73.1 (C-2'), 74.0 (C-3'), 75.0 (C-2”), 77.8 (C-3”), 79.2 (C-4'), 81.8 (C-3), 106.1 (C-1'), 106.8 (C-1”), 122.3 (C-12), 144.5 (C-13), 180.1 (C-28). HRMS: $\mathrm{C}_{40} \mathrm{H}_{64} \mathrm{O}_{12} \mathrm{Na}$ calcd 759.4295; found 759.4266.

\subsection{3- $O$-[ $\beta$-D-glucopyranosyl-(1 $\rightarrow 2)$ - $\alpha$-L-arabinopyranosyl]hederagenin (5). General method}

1. In a typical experiment, $\mathrm{Pd}\left(\mathrm{PPh}_{3}\right)_{4}(0.57 \mathrm{~g}, 0.49 \mathrm{mmol}, 1.0 \mathrm{eq})$ was added to a solution of saponin $41(0.730 \mathrm{~g}, 0.49 \mathrm{mmol})$ in $3 \% \mathrm{KOH}$ in $\mathrm{MeOH}(39 \mathrm{~mL})$. After heating to $60{ }^{\circ} \mathrm{C}$ for $6 \mathrm{~h}$, the reaction was neutralized with Amberlite IR $120\left(\mathrm{H}^{+}\right.$form), filtered and evaporated. The crude residue was taken up in ethanol $(84 \mathrm{~mL})$ and $\mathrm{Pd} / \mathrm{C}(2.51 \mathrm{~g})$ was added. The reaction then was placed under $\mathrm{H}_{2}$. After stirring for $48 \mathrm{~h}$ at room temperature, the mixture was filtered over celite, evaporated, and the 
crude saponin purified by column chromatography $\left(\mathrm{CH}_{2} \mathrm{Cl}_{2} / \mathrm{MeOH} 9: 1\right.$ to 8:2) to give $0.37 \mathrm{~g}$ (82\%) of the desired product $\mathbf{5}$ as an amorphous solid.

General method 2. Use of a catalytic amount of $\mathrm{Pd}\left(\mathrm{PPh}_{3}\right)_{4}$ : in a typical experiment $\operatorname{Pd}\left(\mathrm{PPh}_{3}\right)_{4}$ (0.031 g, $0.03 \mathrm{mmol}, 0.3 \mathrm{eq})$ was added to a solution of saponin $41(0.132 \mathrm{~g}, 0.09 \mathrm{mmol})$ in a $\mathrm{THF} / 3 \% \mathrm{KOH}$ in $\mathrm{MeOH}(1: 1)$ mixture $(6 \mathrm{~mL})$. After heating to $60{ }^{\circ} \mathrm{C}$ for $7 \mathrm{~h}$, the reaction was neutralized with Amberlite IR $120\left(\mathrm{H}^{+}\right.$form), filtered and evaporated. The crude residue was rapidly passed through a silica gel column to remove non-polar impurities $\left(\mathrm{CH}_{2} \mathrm{Cl}_{2} / \mathrm{MeOH}, 9.8: 0.2\right)$ and the partially protected saponin was taken up in ethanol $(7 \mathrm{~mL})$ and $\mathrm{Pd} / \mathrm{C}(0.080 \mathrm{~g})$ was added. The reaction then was placed under $\mathrm{H}_{2}$. After stirring for $24-48 \mathrm{hrs}$ at room temperature, the mixture was filtered over celite, evaporated, and the crude saponin purified by column chromatography $\left(\mathrm{CHCl}_{3} / \mathrm{MeOH} 9: 1\right.$ to 8:2) to give $0.059 \mathrm{~g}(87 \%)$ of the desired product 5 as an amorphous solid. ${ }^{1} \mathrm{H}$ NMR (pyridine-d5) : $\delta 0.89$ (s, 3H, H-25), 0.91 (s, 3H, H-29), 0.97 (s, 6H, H-30, H-26), 0.98-2.15 (m, 22H, H-1, H-2, H-5, H-6, H-7, H-9, H-11, H-15, H-16, H-19, H-21, H-22), 1.00 (s, 3H, H-24), 1.20 (s, 3H, H-27), 3.27 (dd, 1H, $J=13.6, J=3.7$ Hz, H-18), 3.70 (brd, 1H, $J=10.7$ Hz, H-5a'), 3.73 (d, 1H, $J=10.8 \mathrm{~Hz}, \mathrm{H}-23 \mathrm{a}$ ), 3.81 (m, 1H, H-5' '), 4.09 (t, 1H, J=8.0 Hz, H-2' '), 4.16 (dd, 1H, $J=7.5, J=4.5 \mathrm{~Hz}, \mathrm{H}-3), 4.18$ (t, 1H, $J=8.1 \mathrm{~Hz}, \mathrm{H}-3$ ' '), 4.22 (m, 2H, H-23b, H-4' '), 4.27 (dd, 1H, $J$ $=7.0, J=3.7$ Hz, H-3'), 4.28 (m, 1H, H-5b'), 4.32 (m, 1H, H-4'), 4.35 (dd, 1H, $J=11.7, J=4.5 \mathrm{~Hz}$, H-6a' '), 4.47 (dd, 1H, $J=11.7, J=1.6$ Hz, H-6b' '), 4.60 (t, 1H, $J=6.7$ Hz, H-2'), 5.19 (d, 1H, $J=$ $4.6 \mathrm{~Hz}, \mathrm{H}-1$ '), 5.20 (d, 1H, $J=7.0 \mathrm{~Hz}, \mathrm{H}-1$ '’), 5.45 (m, 1H, H-12). ${ }^{13} \mathrm{C}$ NMR (pyridine-d5) : $\delta 13.2$ (C-24), 15.7 (C-25), 17.2 (C-26), 17.8 (C-6), 23.4 (C-16), 23.5 (C-30), 23.5 (C-11), 25.7 (C-2), 25.8 (C-27), 28.0 (C-15), 30.6 (C-20), 32.5 (C-7), 32.9 (C-22), 33.0 (C-29), 34.9 (C-21), 36.6 (C-10), 38.3 (C-1), 39.4 (C-8), 41.6 (C-18), 41.8 (C-14), 43.2 (C-4), 46.3 (C-19), 46.3 (C-17), 47.4 (C-5), 47.8 (C-9), 62.1 (C-6’'), 64.3 (C-23), 64.8 (C-5'), 67.9 (C-4'), 71.0 (C-4'’), 73.2 (C-3'), 75.8 (C2’’), 77.7 (C-3’’), 78.0 (C-5’'), 80.7 (C-2'), 81.8 (C-3), 103.7 (C-1'), 105.4 (C-1'’), 122.2 (C-12), 144.6 (C-13). HRMS: $\mathrm{C}_{41} \mathrm{H}_{66} \mathrm{O}_{13} \mathrm{Na}$ calcd 789.4401; found 789.4415.

\subsection{3- $O$-[ $\beta$-D-glucopyranosyl- $(1 \rightarrow 2)-\beta$-L-arabinopyranosyl]hederagenin $\quad(6)$. Using a} stoichiometric amount of $\mathrm{Pd}\left(\mathrm{PPh}_{3}\right)_{4}$ in the general deprotection method 1 described for 5, the deprotection of saponin $39(0.89 \mathrm{~g}, 0.60 \mathrm{mmol})$ gave $0.40 \mathrm{~g}(88 \%)$ of 6 . Use of the catalytic method with $0.145 \mathrm{~g}(0.10 \mathrm{mmol})$ of 39 gave $0.061 \mathrm{~g}(82 \%)$ of $\mathbf{6}$. $[\alpha]_{\mathrm{D}}=+74.6^{\circ}(c 0.5$, pyridine $)$. Selected NMR data: ${ }^{1} \mathrm{H}$ NMR (pyridine-d5) : $\delta 0.83$ (s, 3H, H-25), 0.85 (s, 3H, H-24), 0.92 (s, 3H, H-29), 0.98 (s, 6H, H-26, H-30), 1.20 (s, 3H, H-27), 3.27 (dd, 1H, J=13.7, J=3.6 Hz, H-18), 3.68 (d, 1H, $J=10.6 \mathrm{~Hz}, \mathrm{H}-23 \mathrm{a}$ ), 3.77 (ddd, $1 \mathrm{H}, J=8.8, J=5.4, J=2.6 \mathrm{~Hz}, \mathrm{H}-5$ ' '), 3.94 (d, 1H, $J=10.8 \mathrm{~Hz}, \mathrm{H}-$ 23b), 4.06 (dd, 1H, $J=12.0, J=1.8 \mathrm{~Hz}, \mathrm{H}-5 \mathrm{a}$ '), 4.12 (dd, $1 \mathrm{H}, J=9.2, J=7.8 \mathrm{~Hz}, \mathrm{H}-2$ '”), 4.15 (m, 1H, H-4' '), 4.16 (m, 1H, H-3' '), 4.23 (dd, 1H, $J=11.8, J=4.4$ Hz, H-3), 4.30 (dd, 1H, $J=11.7, J=$ 
5.4 Hz, H-6a' '), 4.39 (m, 1H, H-4'), 4.44 (m, 2H, H-6b', H-5b'), 4.65 (dd, 1H, J= 9.9, J=3.3 Hz, H-3'), 4.73 (dd, 1H, J= 9.9, J=3.2 Hz, H-2'), 5.12 (d, 1H, J= 7.5 Hz, H-1' '), 5.45 (m, 1H, H-12), $5.82\left(\mathrm{~d}, 1 \mathrm{H}, J=3.3 \mathrm{~Hz}, \mathrm{H}-1\right.$ '). ${ }^{13} \mathrm{C}$ NMR (pyridine-d5) : $\delta 13.8(\mathrm{C}-24), 15.6(\mathrm{C}-25), 17.2(\mathrm{C}-26)$, 22.2 (C-2), 23.5 (C-30), 25.8 (C-27), 33.0 (C-29), 62.4 (C-6’’), 64.2 (C-23), 64.3 (C-5'), 69.2 (C3'), 70.0 (C-4'), 71.3 (C-4’'), 75.2 (C-2'’), 77.3 (C-3), 77.9 (C-3'’), 77.9 (C-5'’), 79.9 (C-2'), 97.7 (C-1'), 106.0 (C-1'’), 122.2 (C-12), 144.6 (C-13). HRMS: $\mathrm{C}_{41} \mathrm{H}_{66} \mathrm{O}_{13} \mathrm{Na}$ calcd 789.4401; found 789.4374 .

4.32. 3- $O$-[ $\beta$-D-glucopyranosyl-( $(1 \rightarrow 3)$ - $\alpha$-L-arabinopyranosyl]hederagenin $\quad(7)$. Using a stoichiometric amount of $\mathrm{Pd}\left(\mathrm{PPh}_{3}\right)_{4}$ in the general deprotection method 1 described for $\mathbf{5}$, the deprotection of saponin $36(0.67 \mathrm{~g}, 0.43 \mathrm{mmol})$ gave $0.27 \mathrm{~g}(78 \%)$ of 7. Use of the catalytic method with $0.060 \mathrm{~g}(0.04 \mathrm{mmol})$ of 36 gave $0.026 \mathrm{~g}(84 \%)$ of 7. $[\alpha]_{\mathrm{D}}=+46.8^{\circ}(c 0.5$, pyridine). Selected NMR data: ${ }^{1} \mathrm{H}$ NMR (pyridine-d5) : $\delta 0.90$ (s, 6H, H-25, H-24), 0.91 (s, 3H, H-29), 0.98 (s, 3H, H30), 0.99 (s, 3H, H-26), 1.24 (s, 3H, H-27), 3.27 (dd, 1H, $J=13.8, J=4.2, \mathrm{H}-18$ ), 3.67 (brd, 1H, $J=$ 12.0, H-5a'), 3.70 (d, 1H, $J=11.0, \mathrm{H}-23 \mathrm{a}), 4.00$ (ddd, 1H, $J=9.5, J=5.3, J=1.9, \mathrm{H}-5$ ' '), 4.07 (t, $1 \mathrm{H}, J=8.4$, H-2' '), 4.15 (m, 1H, H-3'), 4.18 (t, 1H, J=9.1, H-4' '; m, 1H, H-5b'), 4.26 (m, 1H, H3), 4.27 (t, 1H, $J=8.9, \mathrm{H}-3$ ' '), 4.32 (d, 1H, $J=11.4, \mathrm{H}-23 \mathrm{~b}$ ), 4.33 (brd, 1H, $J=11.8, \mathrm{H}-6 \mathrm{a}$ ' '), 4.44 (m, 1H, H-4'), 4.54 (dd, 1H, $J=11.8, J=1.8, \mathrm{H}-6 \mathrm{~b}^{\prime}$ '), 4.58 (dd, 1H, $J=9.0, J=7.8, \mathrm{H}-2$ '), 5.01 (d, $1 \mathrm{H}, J=7.5, \mathrm{H}-1$ '), 5.29 (d, 1H, $J=7.8, \mathrm{H}-1$ '”), 5.47 (m, 1H, H-12). ${ }^{13} \mathrm{C}$ NMR (pyridine- $d_{5}$ ) : $\delta 14.5$ (C-24), 16.8 (C-25), 18.3 (C-26), 24.6 (C-30), 26.9 (C-27), 27.0 (C-2), 34.0 (C-29), 63.2 (C-6’'), 64.8 (C-23), 67.7 (C-5'), 69.9 (C-4'), 72.2 (C-4’’), 72.6 (C-2'), 76.2 (C-2’’), 78.8 (C-3’'), 79.3 (C5'’), 82.6 (C-3), 84.8 (C-3'), 106.7 (C-1'’), 107.2 (C-1'’), 123.3 (C-12), 145.6 (C-13). HRMS: $\mathrm{C}_{41} \mathrm{H}_{66} \mathrm{O}_{13} \mathrm{Na}$ calcd 789.4401; found 789.4391.

3-O-[ $\beta$-D-glucopyranosyl-( $1 \rightarrow 4)$ - $\alpha$-L-arabinopyranosyl]hederagenin $(8)$. Using a stoichiometric amount of $\mathrm{Pd}\left(\mathrm{PPh}_{3}\right)_{4}$ in the general deprotection method 1 described for $\mathbf{5}$, the deprotection of saponin $37(0.80 \mathrm{~g}, 0.50 \mathrm{mmol})$ gave $0.34 \mathrm{~g}(82 \%)$ of 8 . Use of the catalytic method with $0.122 \mathrm{~g}$ $(0.08 \mathrm{mmol})$ of 37 gave $0.041 \mathrm{~g}(65 \%)$ of $\mathbf{8}$. $[\alpha]_{\mathrm{D}}=+35.5^{\circ}\left(c 0.53, \mathrm{CH}_{3} \mathrm{OH}\right)$. Selected NMR data: ${ }^{1} \mathrm{H}$ NMR (pyridine-d5) : $\delta 0.89$ (s, 3H, H-24), 0.91 (s, 3H, H-29), 0.91 (s, 3H, H-25), 0.98 (s, 3H, H30), 0.99 (s, 3H, H-26), 1.23 (s, 3H, H-27), 3.27 (dd, 1H, J=13.6, J=3.6 Hz, H-18), 3.67 (d, 1H, $J$ = 10.6 Hz, H-23a), 3.68 (brd, 1H, J=11.8 Hz, H-5a'), 3.94 (m, 1H, H-5' '), 4.05 (m, 1H, H-2' '), 4.10 (dd, 1H, J = 9.2, J=3.4 Hz, H-3'), 4.21 (m, 1H, H-3), 4.22 (m, 2H, H-3', , H-4'’), 4.27 (d, 1H, $J=11.0 \mathrm{~Hz}, \mathrm{H}-23 \mathrm{~b}), 4.32$ (m, 1H, H-4'), 4.35 (dd, 1H, $J=12.0, J=5.3 \mathrm{~Hz}, \mathrm{H}-6 \mathrm{a}$ ' '), 4.41 (dd, 1H, $J$ $=8.9, J=7.6 \mathrm{~Hz}, \mathrm{H}-2$ '), 4.44 (m, 1H, H-5b'), 4.51 (dd, 1H, $J=11.9, J=1.8 \mathrm{~Hz}, \mathrm{H}-6 \mathrm{~b}$ ' ), 4.94 (d, $\left.1 \mathrm{H}, J=7.3 \mathrm{~Hz}, \mathrm{H}-1^{\prime}\right), 5.23$ (d, $1 \mathrm{H}, J=7.8 \mathrm{~Hz}, \mathrm{H}-1{ }^{\prime}$ '), 5.44 (m, $\left.1 \mathrm{H}, \mathrm{H}-12\right) .{ }^{13} \mathrm{C}$ NMR (pyridine- $d_{5}$ ) : 


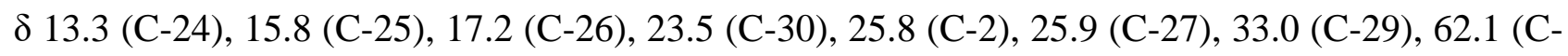
6'’), 63.9 (C-23), 66.0 (C-5'), 70.9 (C-4'’), 73.2 (C-2'), 74.2 (C-3'), 75.3 (C-2'’), 77.9 (C-3'’), 78.3 (C-5'”), 79.4 (C-4'), 81.8 (C-3), 106.1 (C-1'), 106.4 (C-1'’), 122.2 (C-12), 144.6 (C-13). HRMS: $\mathrm{C}_{41} \mathrm{H}_{66} \mathrm{O}_{13} \mathrm{Na}$ calcd 789.4401; found 789.4409.

The saponins 1-8 were treated with excess diazomethane ${ }^{26}$ to give their corresponding methyl esters in quantitative yields.

4.34. Methyl 3- $O$-[ $\beta$-D-xylopyranosyl- $(1 \rightarrow 2)$ - $\alpha$-L-arabinopyranosyl $]$ hederagenate $(1 \mathrm{a}) .[\alpha]_{\mathrm{D}}=$ $+39.0^{\circ}$ (c 0.5, pyridine). ${ }^{1} \mathrm{H}$ NMR $\left(\mathrm{CD}_{3} \mathrm{OD}\right): \delta 0.72$ (s, 3H, H-24), 0.77 (s, 3H, H-26), $0.93(\mathrm{~s}, 3 \mathrm{H}$, H-29), 0.96 (s, 3H, H-30), 0.97-2.06 (m, 22H, H-1, H-2, H-5, H-6, H-7, H-9, H-11, H-15, H-16, H19, H-21, H-22), 1.00 (s, 3H, H-25), 1.19 (s, 3H, H-27), 2.89 (dd, 1H, J= 13.7, J= 3.9 Hz, H-18), 3.18 (dd, 1H, $J=11.1, J=10.7 \mathrm{~Hz}, \mathrm{H}-5 ”), 3.23$ (dd, 1H, $J=9.1, J=7.7 \mathrm{~Hz}, \mathrm{H}-2 ”), 3.26$ (d, 1H, $J=$ $10.1 \mathrm{~Hz}, \mathrm{H}-23$ ), 3.33 (m, 1H, H-3”), 3.48 (ddd, 1H, $J=10.2, J=8.9, J=5.4 \mathrm{~Hz}, \mathrm{H}-4$ "), 3.54 (dd, $1 \mathrm{H}, J=13.2, J=2.6 \mathrm{~Hz}, \mathrm{H}-5$ ') $3.61(\mathrm{dd}, 1 \mathrm{H}, J=12.3, J=4.8 \mathrm{~Hz}, \mathrm{H}-3), 3.64$ (s, 3H, OCH $\mathrm{H}_{3}, 3.72$ (m, 1H, H-23), 3.74 (m, 1H, H-3'), 3.75 (m, 1H, H-2'), 3.84 (m, 3H, H-5’, H-4', H-5'), 4.48 (d, 1H, $J=6.4 \mathrm{~Hz}, \mathrm{H}-1$ '), 4.51 (d, 1H, $J=7.6 \mathrm{~Hz}, \mathrm{H}-1$ ”), 5.27 (m, 1H, H-12). ${ }^{13} \mathrm{C}$ NMR $\left(\mathrm{CD}_{3} \mathrm{OD}\right): \delta 11.6$ (C-24), 14.9 (C-25), 16.2 (C-26), 17.3 (C-6), 22.5 (C-30), 22.6 (C-16), 23.1 (C-11), 25.0 (C-27), 25.0 (C-2), 27.3 (C-15), 30.1 (C-20), 31.8 (C-7), 32.1 (C-29), 32.1 (C-22), 33.3 (C-21), 36.2 (C-10), 38.0 (C-1), 39.1 (C-8), 41.3 (C-18), 41.4 (C-14), 42.6 (C-4), 45.6 (C-19), 46.4 (C-5), 46.6 (C-17), 47.5 (C-9), $50.7\left(\mathrm{OCH}_{3}\right), 62.8$ (C-23), 64.7 (C-5'), 65.7 (C-5”), 68.0 (C-4'), 69.7 (C-4”), 72.6 (C3’), 74.5 (C-2”), 76.4 (C-3”), 79.6 (C-2'), 81.5 (C-3), 103.3 (C-1'), 104.8 (C-1”), 122.3 (C-12), 143.6 (C-13), 178.6 (C-28). HRMS: $\mathrm{C}_{41} \mathrm{H}_{66} \mathrm{O}_{12} \mathrm{Na}$ calcd 773.4452; found 773.4425.

4.35. Methyl 3- $O$-[ $\beta$-D-xylopyranosyl- $(1 \rightarrow 2)$ - $\beta$-L-arabinopyranosyl $]$ hederagenate $(2 \mathrm{a}) .[\alpha]_{\mathrm{D}}=$ $+87.2^{\circ}$ (c 0.5, pyridine). Selected NMR data: ${ }^{1} \mathrm{H}$ NMR $\left(\mathrm{CD}_{3} \mathrm{OD}\right): \delta 0.69$ (s, 3H, H-24), 0.77 (s, 3H, H-26), 0.93 (s, 3H, H-30), 0.96 (s, 3H, H-29), 1.00 (s, 3H, H-25), 1.19 (s, 3H, H-27), 2.89 (dd, $1 \mathrm{H}, J=13.7, J=4.1 \mathrm{~Hz}, \mathrm{H}-18), 3.21$ (dd, 1H, $J=11.3, J=10.4 \mathrm{~Hz}, \mathrm{H}-5$ ”), 3.27 (dd, 1H, $J=9.0, J=$ 7.4 Hz, H-2”), 3.33 (m, 1H, H-23), 3.34 (t, 1H, J= 8,9 Hz, H-3”), 3.47 (d, 1H, J=11.2 Hz, H-23), 3.50 (m, 1H, H-4”), 3.59 (dd, 1H, $J=12.4, J=1.2 \mathrm{~Hz}, \mathrm{H}-5$ '), 3.64 (s, 3H, OCH $H_{3}$, 3.65 (dd, 1H, $J=$ $11.4, J=4.7 \mathrm{~Hz}, \mathrm{H}-3), 3.83$ (dd, $\left.1 \mathrm{H}, J=9.3, J=3.4 \mathrm{~Hz}, \mathrm{H}-2^{\prime}\right), 3.88$ (dd, $1 \mathrm{H}, J=11.4, J=5.3 \mathrm{~Hz}, \mathrm{H}-$ 5”), 3.94 (m, 1H, H-4'), 3.95 (m, 1H, H-3'), 3.98 (brd, 1H, J=12.2 Hz, H-5'), 4.41 (d, 1H, J= 7.4 $\mathrm{Hz}, \mathrm{H}-1$ ”), 5.14 (d, 1H, J= $3.4 \mathrm{~Hz}, \mathrm{H}-1$ '), 5.28 (m, 1H, H-12). ${ }^{13} \mathrm{C}$ NMR (CD $\left.{ }_{3} \mathrm{OD}\right): \delta 12.4(\mathrm{C}-24)$, 14.8 (C-25), 16.2 (C-26), 21.6 (C-2), 22.5 (C-30), 25.0 (C-27), 32.1 (C-29), $50.7\left(\mathrm{OCH}_{3}\right), 63.2$ (C5’), 63.5 (C-23), 65.4 (C-5”), 68.1 (C-3'), 69.4 (C-4'), 69.7 (C-4”), 73.9 (C-2”), 76.3 (C-3”), 77.2 
(C-3), 78.4 (C-2'), 97.0 (C-1'), 105.4 (C-1’), 122.3 (C-12), 143.6 (C-13), 178.6 (C-28). HRMS: $\mathrm{C}_{41} \mathrm{H}_{66} \mathrm{O}_{12} \mathrm{Na}$ calcd 773.4452; found 773.4456.

4.36. Methyl 3- $O$ - $[\beta$-D-xylopyranosyl-(1 $\rightarrow 3)$ - $\alpha$-L-arabinopyranosyl $]$ hederagenate $(3 \mathrm{a}) .[\alpha]_{\mathrm{D}}=$ $+49.0^{\circ}$ ( $c$ 0.5, pyridine). Selected NMR data: ${ }^{1} \mathrm{H}$ NMR $\left(\mathrm{CD}_{3} \mathrm{OD}\right): \delta 0.74(\mathrm{~s}, 3 \mathrm{H}, \mathrm{H}-24), 0.77(\mathrm{~s}, 3 \mathrm{H}$, H-26), 0.93 (s, 3H, H-29), 0.96 (s, 3H, H-30), 1.00 (s, 3H, H-25), 1.19 (s, 3H, H-27), 2.89 (dd, 1H, $J=13.5, J=3.4 \mathrm{~Hz}, \mathrm{H}-18), 3.23$ (dd, 1H, $J=11.0, J=10.7$ Hz, H-5”), 3.31 (m, 1H, H-23), 3.32 (m, 1H, H-2”), 3.36 (t, 1H, J=8.2 Hz, H-3”), 3.52 (m, 1H, H-4”), 3.58 (brd, 1H, J= 12.4 Hz, H-5'), 3.63 (m, 2H, H-3, H-3'), 3.64 ( $\left.\mathrm{s}, 3 \mathrm{H}, \mathrm{OCH}_{3}\right), 3.65$ (m, 1H, H-23), 3.71 (dd, 1H, J=9.3, J= 7.6 Hz, H-2'), 3.87 (dd, 1H, J= 12.4, J=2.0 Hz, H-5'), 3.88 (dd, 1H, J=11.3, J= 5.4 Hz, H-5”), 3.97 (m, 1H, H-4'), 4.36 (d, 1H, J=7.5 Hz, H-1'), 4.53 (d, 1H, J=7.1 Hz, H-1”), 5.27 (m, 1H, H-12). ${ }^{13} \mathrm{C}$ NMR (CD $\left.{ }_{3} \mathrm{OD}\right)$ : $\delta 11.9$ (C-24), 15.0 (C-25), 16.2 (C-26), 22.5 (C-30), 25.1 (C-27), 32.1 (C-29), $50.8\left(\mathrm{OCH}_{3}\right), 63.6$ (C-23), 65.4 (C-5'), 65.5 (C-5”), 68.3 (C-4'), 69.6 (C-4”), 70.7 (C-2'), 73.7 (C2”), 76.0 (C-3”), 82.0 (C-3), 82.2 (C-3'), 104.7 (C-1', C-1”), 122.3 (C-12), 143.6 (C-13), 178.5 (C28). HRMS: $\mathrm{C}_{41} \mathrm{H}_{66} \mathrm{O}_{12} \mathrm{Na}$ calcd 773.4452; found 773.4450 .

4.37. Methyl 3- $O$-[ $\beta$-D-xylopyranosyl-(1 $\rightarrow 4)$ - $\alpha$-L-arabinopyranosyl $]$ hederagenate $(4 a) .[\alpha]_{\mathrm{D}}=$ $+45.2^{\circ}$ (c 0.5, pyridine). Selected NMR data: ${ }^{1} \mathrm{H}$ NMR $\left(\mathrm{CD}_{3} \mathrm{OD}\right): \delta 0.69(\mathrm{~s}, 3 \mathrm{H}, \mathrm{H}-24), 0.73(\mathrm{~s}, 3 \mathrm{H}$, H-26), 0.89 (s, 3H, H-29), 0.92 (s, 3H, H-30), 0.96 (s, 3H, H-25), 1.15 (s, 3H, H-27), 2.85 (dd, 1H, $J=13.7, J=3.8 \mathrm{~Hz}, \mathrm{H}-18), 3.16$ (dd, 1H, $J=11.0, J=10.8 \mathrm{~Hz}, \mathrm{H}-5$ ”), 3.25 (m, 1H, H-2”), 3.26 (d, 1H, $J=11.4$ Hz, H-23), 3.29 (m, 1H, H-3”), 3.46 (m, 1H, H-4”), 3.50 (m, 1H, H-2'), 3.51 (m, 1H, H-5'), 3.53 (m, 1H, H-3'), 3.58 (dd, 1H, J=11.5, J=4.7 Hz, H-3), 3.59 (m, 1H, H-23), 3.60 (s, 3H, $\mathrm{OCH}_{3}$ ), 3.85 (dd, 1H, $J=11.4, J=5.3 \mathrm{~Hz}, \mathrm{H}-5$ '), 3.83 (m, 1H, H-4'), 4.03 (dd, 1H, $J=12.6, J=2.4$ Hz, H-5'), 4.28 (d, 1H, J= 6.4 Hz, H-1'), 4.39 (d, 1H, J= $7.1 \mathrm{~Hz}, \mathrm{H}-1$ '), 5.23 (m, 1H, H-12). ${ }^{13} \mathrm{C}$ NMR (CD $\left.{ }_{3} \mathrm{OD}\right)$ : $\delta 11.9$ (C-24), 14.9 (C-25), 16.2 (C-26), 22.5 (C-30), 25.0 (C-27), 32.1 (C-29), $50.7\left(\mathrm{OCH}_{3}\right), 63.3$ (C-23), 65.1 (C-5’), 65.5 (C-5”), 69.6 (C-4”), 71.9 (C-2'), 73.0 (C-3'), 73.8 (C2”), 76.3 (C-3”), 78.4 (C-4'), 82.0 (C-3), 104.9 (C-1'), 105.6 (C-1”), 122.3 (C-12), 143.6 (C-13), 178.6 (C-28). HRMS: $\mathrm{C}_{41} \mathrm{H}_{66} \mathrm{O}_{12} \mathrm{Na}$ calcd 773.4452; found 773.4441.

4.38. Methyl 3- $O$-[ $\beta$-D-glucopyranosyl- $(1 \rightarrow 2)$ - $\alpha$-L-arabinopyranosyl $]$ hederagenate $(5 a) .[\alpha]_{D}=$ $+39.8^{\circ}$ ( $c$ 0.5, pyridine). Selected NMR data: ${ }^{1} \mathrm{H}$ NMR $\left(\mathrm{CD}_{3} \mathrm{OD}\right): \delta 0.74(\mathrm{~s}, 3 \mathrm{H}, \mathrm{H}-24), 0.77(\mathrm{~s}, 3 \mathrm{H}$, H-26), 0.93 (s, 3H, H-29), 0.96 (s, 3H, H-30), 1.00 (s, 3H, H-25), 1.19 (s, 3H, H-27), 2.89 (dd, 1H, $J=13.6, J=3.9 \mathrm{~Hz}, \mathrm{H}-18$ ), 3.23 (dd, $1 \mathrm{H}, J=8.7, J=7.9 \mathrm{~Hz}, \mathrm{H}-2$ '”), 3.25 (t, 1H, $J=9.1 \mathrm{~Hz}, \mathrm{H}-4$ ' '), 3.28 (m, 1H, H-5' '), 3.32 (m, 1H, H-23a), 3.38 (dd, 1H, $J=9.0, J=8.7$ Hz, H-3'’), 3.54 (dd, 1H, $J=$ $13.3, J=3.3 \mathrm{~Hz}, \mathrm{H}-5 \mathrm{a}$ '), 3.64 (s, 3H, $\left.\mathrm{OCH}_{3} ; \mathrm{m}, 1 \mathrm{H}, \mathrm{H}-3\right), 3.65$ (m, 1H, H-6a' '), 3.67 (d, 1H, $J=$ 
$11.2 \mathrm{~Hz}, \mathrm{H}-23 \mathrm{~b}$ ), 3.77 (dd, 1H, $J=8.1, J=3.1 \mathrm{~Hz}, \mathrm{H}-3$ '), 3.85 (m, 1H, H-4'), 3.86 (m, 3H, H-6b', H-5b', H-2'), 4.56 (d, 1H, J=6.2 Hz, H-1'), 4.63 (d, 1H, J= 7.8 Hz, H-1'”), 5.27 (m, 1H, H-12). ${ }^{13} \mathrm{C}$ NMR (CD $\left.3 \mathrm{OD}\right): \delta 11.9$ (C-24), 14.9 (C-25), 16.2 (C-26), 22.5 (C-30), 24.9 (C-2), 25.0 (C-27), 32.0 (C-29), $50.7\left(\mathrm{OCH}_{3}\right), 61.4$ (C-6’'), 63.3 (C-23), 64.1 (C-5'), 67.6 (C-4'), 70.2 (C-4'’), 72.4 (C3'), 74.5 (C-2'’), 76.5 (C-3'), 76.8 (C-5'), 77.9 (C-2'), 82.2 (C-3), 103.1 (C-1'), 103.2 (C-1'’), 122.3 (C-12), 143.6 (C-13), 178.6 (C-28). HRMS: $\mathrm{C}_{42} \mathrm{H}_{68} \mathrm{O}_{13} \mathrm{Na}$ calcd 803.4558; found 803.4554.

4.39. Methyl 3- $O$-[ $\beta$-D-glucopyranosyl- $(1 \rightarrow 2)$ - $\beta$-L-arabinopyranosyl $]$ hederagenate $(6 \mathbf{a}) .[\alpha]_{\mathrm{D}}=$ $+72.2^{\circ}\left(c\right.$ 0.5, pyridine). Selected NMR data : ${ }^{1} \mathrm{H}$ NMR $\left(\mathrm{CD}_{3} \mathrm{OD}\right): \delta 0.69(\mathrm{~s}, 3 \mathrm{H}, \mathrm{H}-24), 0.77(\mathrm{~s}, 3 \mathrm{H}$, H-26), 0.93 (s, 3H, H-29), 0.96 (s, 3H, H-30), 1.00 (s, 3H, H-25), 1.19 (s, 3H, H-27), 2.90 (dd, 1H, $J=13.8, J=3.9 \mathrm{~Hz}, \mathrm{H}-18), 3.27$ (m, 1H, H-4' '), 3.28 (m, 1H, H-2'’), 3.30 (m, 1H, H-5' '), 3.34 (m, 1H, H-23a), 3.38 (t, 1H, J=8.8 Hz, H-3') ), 3.50 (d, 1H, J=11.1 Hz, H-23b), 3.60 (dd, 1H, J= 12.1, $J=1.6 \mathrm{~Hz}, \mathrm{H}-5 \mathrm{a}$ ') 3.64 (s, 3H, OCH $\left.H_{3}\right), 3.67$ (dd, 1H, $J=11.8, J=5.7 \mathrm{~Hz}, \mathrm{H}-6 \mathrm{a}{ }^{\prime}$ ), 3.72 (dd, 1H, $J=$ 11.6, $J=4.5 \mathrm{~Hz}, \mathrm{H}-3$ ), 3.86 (dd, $1 \mathrm{H}, J=9.5, J=3.3 \mathrm{~Hz}, \mathrm{H}-2$ ') 3.90 (dd, $1 \mathrm{H}, J=11.8, J=2.0 \mathrm{~Hz}, \mathrm{H}-$ 6b' '), 3.94 (m, 1H, H-4'), 3.96 (dd, 1H, $J=9.7, J=3.4$ Hz, H-3'), 3.99 (brd, 1H, $J=12.0 \mathrm{~Hz}, \mathrm{H}-$ $\left.5 b^{\prime}\right), 4.49$ (d, 1H, $J=7.7 \mathrm{~Hz}, \mathrm{H}-1$ '’), 5.28 (m, 1H, H-12; d, 1H, J=3.2 Hz, H-1'). ${ }^{13} \mathrm{C}$ NMR $\left(\mathrm{CD}_{3} \mathrm{OD}\right): \delta 12.4$ (C-24), 14.8 (C-25), 16.2 (C-26), 22.1 (C-2), 22.5 (C-30), 25.0 (C-27), 32.0 (C29), $50.7\left(\mathrm{OCH}_{3}\right), 61.5$ (C-6’'), 63.3 (C-5'), 63.5 (C-23), 68.0 (C-3'), 69.4 (C-4'), 70.3 (C-4’’), 74.2 (C-2’’), 76.5 (C-3'’), 76.6 (C-5'’), 78.0 (C-3), 78.6 (C-2'), 97.8 (C-1'), 104.5 (C-1'’), 122.4 (C-12), 143.6 (C-13), 178.6 (C-28). HRMS: $\mathrm{C}_{42} \mathrm{H}_{68} \mathrm{O}_{13} \mathrm{Na}$ calcd 803.4558; found 803.4554.

4.40. Methyl 3- $O$-[ $\beta$-D-glucopyranosyl- $(1 \rightarrow 3)$ - $\alpha$-L-arabinopyranosyl $]$ hederagenate $(7 \mathrm{a}) .[\alpha]_{\mathrm{D}}=$ $+47.0^{\circ}$ ( $c$ 0.5, pyridine). Selected NMR data : ${ }^{1} \mathrm{H}$ NMR $\left(\mathrm{CD}_{3} \mathrm{OD}\right): \delta 0.84(\mathrm{~s}, 3 \mathrm{H}, \mathrm{H}-24), 0.87(\mathrm{~s}, 3 \mathrm{H}$, H-26), 0.93 (s, 3H, H-29), 0.96 (s, 3H, H-30), 1.00 (s, 3H, H-25), 1.19 (s, 3H, H-27), 2.89 (dd, 1H, $J=13.5, J=4.0 \mathrm{~Hz}, \mathrm{H}-18), 3.30$ (m, 1H, H-5' ), 3.32 (m, 1H, H-2' '), 3.32 (m, 1H, H-23a), 3.37 (t, $1 \mathrm{H}, J=8.5 \mathrm{~Hz}, \mathrm{H}-4$ ' '), 3.40 (t, 1H, $J=8.6 \mathrm{~Hz}, \mathrm{H}-3$ ' '), 3.59 (dd, 1H, $J=12.8, J=1.2 \mathrm{~Hz}, \mathrm{H}-5 \mathrm{a}$ '), 3.64 (s, 3H, $\mathrm{OCH}_{3}$; m, 1H, H-3), 3.65 (m, 1H, H-3' ; d, 1H, J=11.6 Hz, H-23b), 3.71 (dd, 1H, $J=$ $12.1, J=5.4 \mathrm{~Hz}, \mathrm{H}-6 \mathrm{a}^{\prime}$ '; dd, $1 \mathrm{H}, J=9.8, J=7.5 \mathrm{~Hz}, \mathrm{H}-2$ ') 3.85 (dd, $1 \mathrm{H}, J=11.9, J=2.1 \mathrm{~Hz}, \mathrm{H}-$ 6b' '), 3.88 (dd, 1H, J=13.0, J=2.2 Hz, H-5b'), 4.06 (m, 1H, H-4'), 4.37 (d, 1H, J= 7.4 Hz, H-1'), 4.56 (d, 1H, $J=7.7 \mathrm{~Hz}, \mathrm{H}-1$ '’), 5.27 (brt, $1 \mathrm{H}, J=3.3 \mathrm{~Hz}, \mathrm{H}-12) .{ }^{13} \mathrm{C}$ NMR $\left(\mathrm{CD}_{3} \mathrm{OD}\right): \delta 11.9$, (C24), 15.0 (C-25), 16.2 (C-26), 22.5 (C-30), 24.8 (C-2), 25.0 (C-27), 32.0 (C-29), $50.7\left(\mathrm{OCH}_{3}\right), 60.9$ (C-6’'), 63.7 (C-23), 65.4 (C-5'), 68.1 (C-4'), 69.7 (C-4'’), 70.6 (C-2'), 73.9 (C-2'’), 76.2 (C-3'’), 76.5 (C-5'’), 82.0 (C-3), 82.8 (C-3'), 104.1 (C-1'’), 104.7 (C-1'), 122.3 (C-12), 143.6 (C-13), 178.6 (C-28). HRMS: $\mathrm{C}_{42} \mathrm{H}_{68} \mathrm{O}_{13} \mathrm{Na}$ calcd 803.4558; found 803.4534. 
4.41. Methyl 3- $O$-[ $\beta$-D-glucopyranosyl- $(1 \rightarrow 4)$ - $\alpha$-L-arabinopyranosyl $]$ hederagenate $(8 \mathrm{a}) .[\alpha]_{\mathrm{D}}=$ $+22.1^{\circ}$ ( $c$ 0.5, pyridine). Selected NMR data : ${ }^{1} \mathrm{H}$ NMR $\left(\mathrm{CD}_{3} \mathrm{OD}\right): \delta 0.73$ (s, 3H, H-24), $0.77(\mathrm{~s}, 3 \mathrm{H}$, H-26), 0.93 (s, 3H, H-29), 0.96 (s, 3H, H-30), 1.00 (s, 3H, H-25), 1.19 (s, 3H, H-27), 2.89 (dd, 1H, $J=13.7, J=3.9$ Hz, H-18), 3.30 (m, 2H, H-2', H-5' '), 3.32 (m, 1H, H-23a), 3.33 (m, 1H, H-4' '), 3.37 (t, 1H, J = 8.6 Hz, H-3'’), 3.56 (m, 3H, H-2', H-3', H-5a'), 3.63 (m, 1H, H-3), 3.64 (s, 3H, $\mathrm{OCH}_{3}$; d, 1H, $J=11.2 \mathrm{~Hz}, \mathrm{H}-23 \mathrm{~b}$ ), 3.68 (dd, 1H, $J=12.0, J=5.3 \mathrm{~Hz}, \mathrm{H}-6 \mathrm{a}{ }^{\prime}$ '), 3.87 (dd, 1H, $J=$ $12.1, J=2.1 \mathrm{~Hz}, \mathrm{H}-6 \mathrm{~b}^{\prime \prime}$ ), 3.93 (m, 1H, H-4'), 4.20 (dd, 1H, $J=12.7, J=2.4 \mathrm{~Hz}, \mathrm{H}-5 \mathrm{~b}^{\prime}$ ), 4.33 (d, $\left.1 \mathrm{H}, J=6.8 \mathrm{~Hz}, \mathrm{H}-1^{\prime}\right), 4.49$ (d, $1 \mathrm{H}, J=7.6 \mathrm{~Hz}, \mathrm{H}-1{ }^{\prime}$ '), 5.27 (m, 1H, H-12). ${ }^{13} \mathrm{C}$ NMR $\left(\mathrm{CD}_{3} \mathrm{OD}\right): \delta$ 11.9 (C-24), 14.9 (C-25), 16.2 (C-26), 22.5 (C-30), 24.7 (C-2), 25.0 (C-27), 32.1 (C-29), 50.7 $\left(\mathrm{OCH}_{3}\right), 61.1$ (C-6') 63.3 (C-23), 65.1 (C-5'), 69.8 (C-4'’), 71.9 (C-2'), 73.0 (C-3'), 73.9 (C-2'’), 76.4 (C-3'’), 76.5 (C-5'), 78.5 (C-4'), 81.9 (C-3), 104.8 (C-1'), 104.9 (C-1'’), 122.3 (C-12), 143.6 (C-13), 178.6 (C-28). HRMS: $\mathrm{C}_{42} \mathrm{H}_{68} \mathrm{O}_{13} \mathrm{Na}$ calcd 803.4558; found 803.4531.

\section{Acknowledgements}

The authors would like to thank the CNRS (Centre National de Recherche Scientifique) and the French Research Ministry for financial support as well as the Ph. D. scholarship for MC.

\section{References and notes}

1. Hostettmann, K.; Marston, A. Saponins; Cambridge University Press: Cambridge, 1995.

2. Sparg, S.G.; Light, M.E.; van Staden, J. J. Ethnopharmacol. 2004, 94, 219-243.

3. Francis, G.; Kerem, Z.; Makkar, H.P.S.; Becker, K. Br. J. Nutr., 2002, 88, 587-605.

4. Chwalek, M.; Plé, K.; Voutquenne-Nazabadioko, L. Chem. Pharm. Bull. 2004, 52, 965-971.

5. Plé, K.; Chwalek, M.; Voutquenne-Nazabadioko, L. Eur. J. Org. Chem. 2004, 1588-1603.

6. (a) Higuchi, R.; Kawasaki, T. Chem.Pharm. Bull. 1976, 24, 1021-1032. (b) Jayasinghe, U.L.B.; Fujimoto, Y.; Hostettmann, K. Nat. Prod. Lett., 1988, 12, 135-138. (c) Joshi, B.S.; Moore, K.M.; Pelletier, S.W. J. Nat. Prod. 1992, 55, 1468-1476. (d) Zhu, N.; Sheng, S.; Sang, S.; Jhoo, J-W.; Bai, N.; Karwe, M.V.; Rosen, R.T.; Ho, C-T. J. Agric. Food Chem. 2002, 50, 865-867.

7. (a) Gopalsamy, N.; Gueho, J.; Julien, H.R.; Owadally, A.W.; Hostettmann, K. Phytochemistry, 1990, 29, 793-795. (b) Domon, B.; Hostettmann, K. Helv. Chem. Acta. 1983, 66, 422-428.

8. Kawai, H.; Kuroyanagi, M.; Umehara, K.; Ueno, A.; Satake, M. Chem.Pharm. Bull. 1988, 36, 4769-4775.

9. Jung, H-J.; Lee, C.O.; Lee, K-T.; Choi, J.; Park, H-J. Biol. Pharm. Bull. 2004, 27, 744-747.

10. Pellissier, H. Tetrahedron, 2004, 60, 5123-5162. 
11. (a) Yu, B.; Xie, J.; Deng, S.; Hui, Y. J. Am. Chem. Soc. 1999, 121, 12196-12197. (b) Seebacher W.; Haslinger, E.; Rauchensteiner, K.; Jurenitsch, J.; Presser, A.; Weis, R. Monatsh. Chem. 1999, 130, 887-897. (c) Seebacher W.; Weis, R.; Jurenitsch, J.; Rauchensteiner, K.; Haslinger, E. Monatsh. Chem. 1999, 130, 1383-1391. (d) Seebacher W.; Weis, R.; Jurenitsch, J.; Rauchensteiner, K.; Haslinger, E. Monatsh. Chem. 2000, 131, 985-996. (e) Sun, J. ; Han, X. ; Yu, B. Carbohydr. Res. 2003, 338, 827-833. (f) Peng, W.; Sun, J.; Lin, F.; Han, X.; Yu, B. Synlett, 2004, 259-262.

12. (a) Takechi, M.; Tanaka, Y. Phytochemistry, 1993, 32, 1173-1175. (b) Saito, S.; Sasaki, Y.; Kuroda, K.; Hayashi,Y.; Sumita, S.; Nagamura, Y.; Nishida, K.; Ishiguro, I. Chem.Pharm. Bull. 1993, 41, 539-543. (c) Ullah, N.; Seebacher, W.; Weis, R.; Jurenitsch, J.; Rauchensteiner, K.; Haslinger, E. Monatsh. Chem. 2000, 131, 787-794.

13. Takechi, M.; Tanaka, Y. Phytochemistry, 1993, 34, 675-677.

14. Levy, M.; Zehavi, U.; Naim, M.; Polacheck, I. Carbohydr. Res. 1989, 193, 115-123.

15. (a) Suhr, R.; Thiem, J. J. Carbohydr. Chem. 2004, 23, 261-276. (b) Deng, L.; Wu, H.; Yu, B.; Jiang, M.; Wu, J. Biorg. Med. Chem. Lett. 2004, 14, 2781-2785. (c) Yu, W.; Jin, Z. J. Am. Chem. Soc. 2002, 124, 6576-6583. (d) Ma, X.; Yu, B.; Hui, Y.; Miao, Z.; Ding, J. Carbohydr. Res. 2001, 334, 159-164. (e) Deng, S.; Yu, B.; Lou, Y.; Hui, Y. J. Org. Chem. 1999, 64, 202-208.

16. Ma, X.; Yu, B.; Hui, Y.; Xiao, D.; Ding, J. Carbohydr. Res. 2000, 329, 495-505.

17. Liptak, A.; Szurmai, Z.; Nanasi, P.; Neszmelyi, A. Tetrahedron, 1982, 38, 3489-3497.

18. Mukhopadhyay, B.; Field, R.A. Carbohydr. Res. 2004, 339, 1285-1291.

19. Chen, L. ; Kong, F. Carbohydr. Res. 2002, 337, 2335-2341.

20. Ziegler, T.; Jurisch, C. Tetrahedron : Asymmetry, 2000, 11, 3403-3418.

21. Verduyn, R.; Douwes, M.; van der Klein, P.A.M.; Mösinger, E.M.; van der Marel, G.A.; van Boom, J.H. Tetrahedron, 1993, 49, 7301-7316.

22. Thijssen, M.; Halkes, K.; Kamerling, J.; Vliegenthart, J. Biorg. Med. Chem. 1994, 2, 13091317.

23. Schmidt, R.R.; Behrendt, M.; Toepfer, A. Synlett, 1990, 694-696.

24. Guibe, F. Tetrahedron, 1998, 54, 2967-3042.

25. Yamada, M.; Watanabe, S.; Hoshino, O.; Ishizaki, M.; Hayashida, M.; Tanaka, A.; Hara, H. Chem. Pharm. Bull. 2003, 51, 1220-1221.

26. Lombardi, P. Chem. Ind. (London), 1990, 21, 708.

27. Suhr, R.; Pfefferkorn, P.; Weingarten, S.; Thiem, J. Org. Biomol. Chem. 2003, 1, 4373-4379. 